\title{
OPTIMIZATION OF GAN LASER DIODES USING \\ 1D AND 2D OPTICAL SIMULATIONS
}

\author{
A Thesis \\ presented to \\ the Faculty of California Polytechnic State University, \\ San Luis Obispo
}

\author{
In Partial Fulfillment \\ of the Requirements for the Degree \\ Master of Science in Electrical Engineering
}

by

Sean Richard Keali’i Jobe

March 2009 
(C) 2009

Sean Richard Keali'i Jobe

ALL RIGHTS RESERVED 


\section{APPROVAL PAGE}

TITLE:

OPTIMIZATION OF GAN LASER DIODES USING 1D AND 2D OPTICAL SIMULATIONS

AUTHOR:

Sean Richard Keali'i Jobe

DATE SUBMITTED:

March 2009

COMMITTEE CHAIR:

Xiaomin Jin, Professor

COMMITTEE MEMBER: Xiao-Hua Yu, Professor

COMMITTEE MEMBER: $\quad$ Samuel Agbo, Professor 


\section{ABSTRACT \\ OPTIMIZATION OF GAN LASER DIODES USING \\ 1D AND 2D OPTICAL SIMULATIONS \\ Sean Richard Keali'i Jobe}

This paper studies the optical properties of a GaN Laser Diode (LD). Through simulation, the GaN LD is optimized for the best optical confinement factor. It is found that there are optimal thicknesses of each layer in the diode that yield the highest optical confinement factor. There is a strong relationship between the optical confinement factor and lasing threshold—a higher optical confinement factor results in a lower lasing threshold. Increasing optical confinement improves lasing efficiency. Blue LDs are important to the future of lighting sources as they represent the final color in the RGB spectrum that does not have a high efficiency solution. The modeled GaN LD emits blue light at around $\sim 450 \mathrm{~nm}$. Each layer of the GaN LD is drawn in a model simulation program called LaserMOD created by RSOFT Design Group, Inc. By properly modifying the properties of each layer, an accurate model of the GaN LD is created and then simulated. This paper describes the steps taken to properly model and optimize the GaN LD in the 1D and 2D models. 


\section{ACKNOWLEDGMENTS}

I would like to thank Dr. Xiaomin Jin for introducing me to GaN laser diodes and supporting me through my research and optical simulations. I would also like to thank my

family for their gracious support and encouragement throughout my educational endeavors. 


\section{TABLE OF CONTENTS}

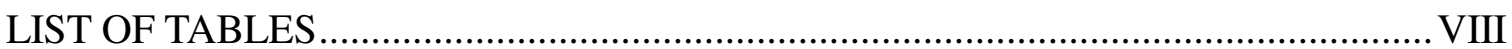

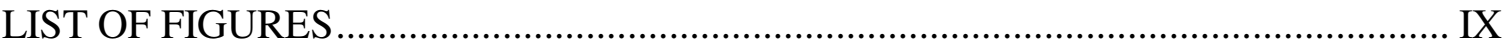

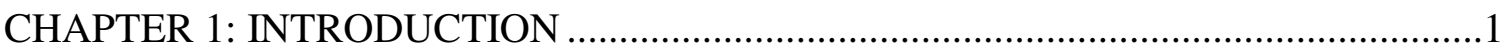

1.1 Lasers Diodes, Light Emitting Diodes and Light .................................................... 1

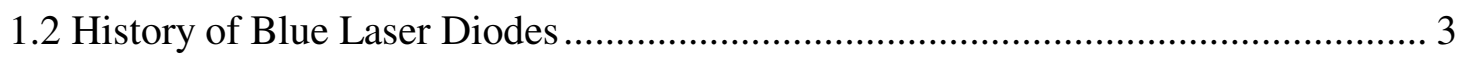

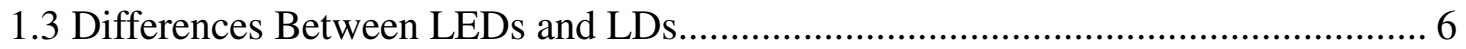

1.4 Laser Diode Structure: Further Into How an LD Works …………………….......... 6

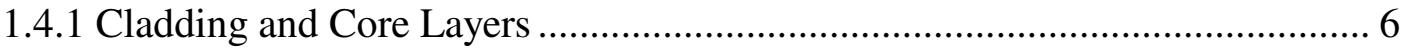

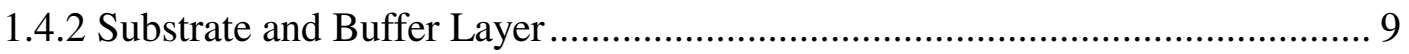

1.4.3 Quantum Wells (QW) Layer.................................................................. 9

1.4.4 Electron Block (e-Block) Layer....................................................................11

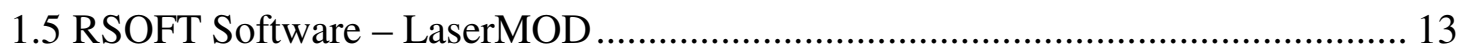

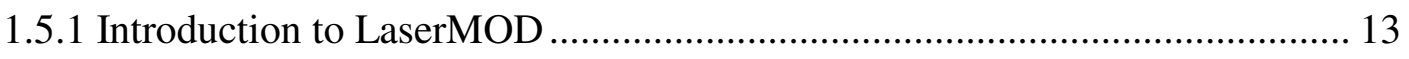

1.5.2 Mathematical Model - 8x8 Kronig-Penney (KP) Model ................................ 13

1.5.3 Optical Confinement Factor (OCF)........................................................... 14

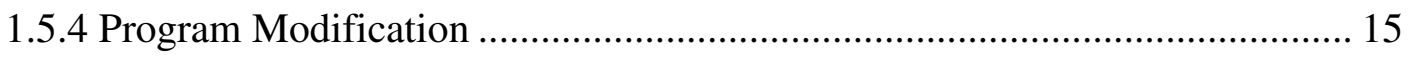

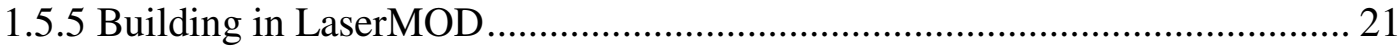

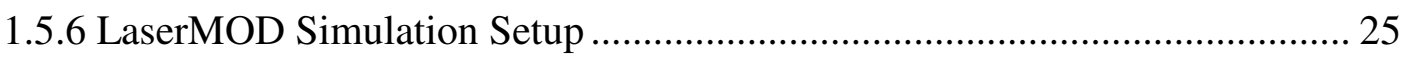

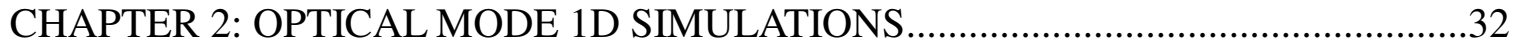

2.1 Optical Modes in 1D Simulations of GaN LDs ..................................................... 32

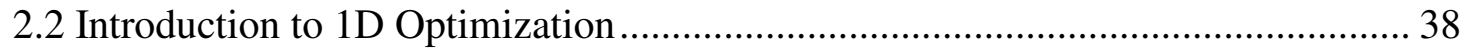

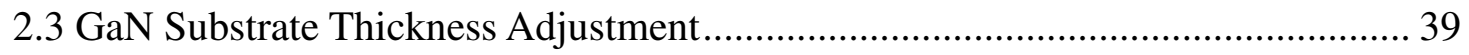


2.4 n-AlGaN (n-SL) and p-AlGaN (p-SL) Cladding Thickness Adjustment .............. 41

2.5 n-GaN and p-GaN Cladding Thickness Adjustment ....................................... 47

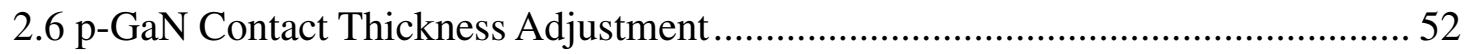

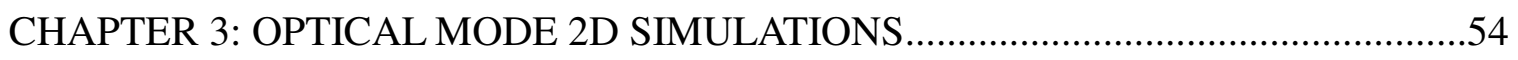

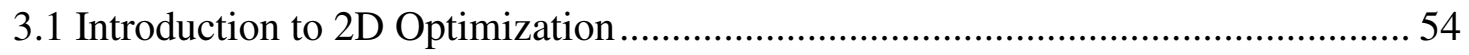

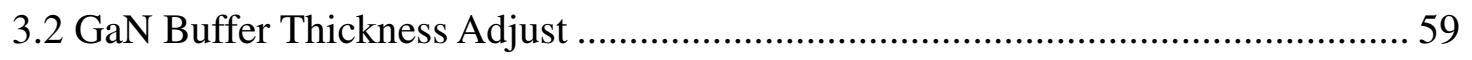

3.3 n-AlGaN and p-AlGaN SL Cladding Thickness Adjust....................................... 62

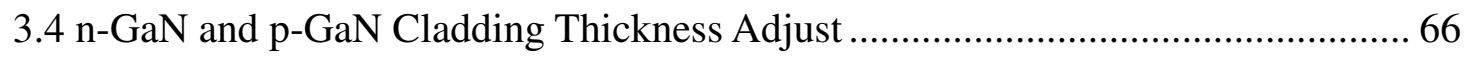

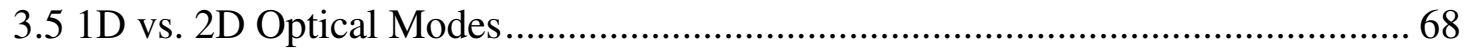

CHAPTER 4: OPTICAL POWER VS. CURRENT ANALYSIS IN 1D

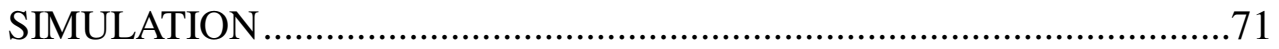

4.1 Light vs. Current Plot of Original 1D Design ................................................. 71

4.2 Light vs. Current Plot of Optimized 1D Design.............................................. 74

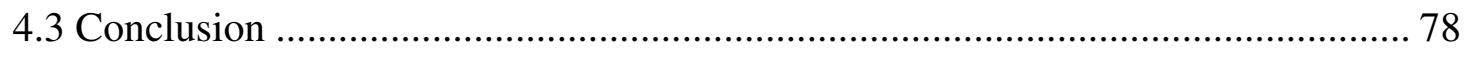

4.4 Errors and Corrections and Future Work ........................................................ 79

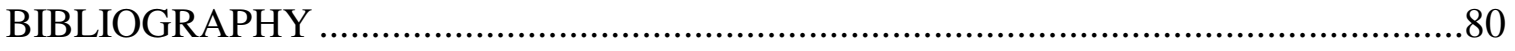

APPENDIX 83 


\section{LIST OF TABLES}

Table 1-1: Materials of the LD and corresponding refractive indices............................ 18

Table 1-2: Laser diode layer structure and parameters............................................... 22

Table 4-1: Table of lasing threshold currents for different quantum wells ..................... 74

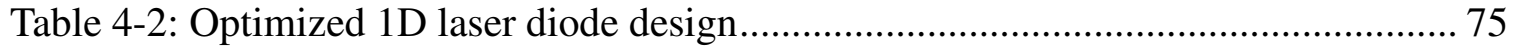




\section{LIST OF FIGURES}

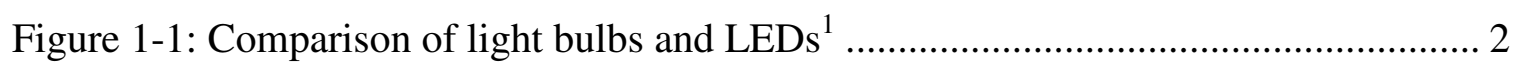

Figure 1-2: Single Heterostructure Laser ............................................................. 7

Figure 1-3: (a) Multiple Quantum Well and (b) Super-Lattice structures....................... 8

Figure 1-4: Summary of advantages by implementing QW structures. ..........................11

Figure 1-5: AlGaN current-blocking layer in an AlGaN/GaN/GaInN MQW LED

structure. (a) Band diagram without doping. (b) Band diagram with doping.

The $\mathrm{Al}$ content in the electron-blocking layer is higher than in the p-type confinement layer. .12

Figure 1-6: RSOFT LaserMOD software. A GaN Laser Diode is currently drawn. ......... 15

Figure 1-7: Window to edit individual bulk layer................................................. 16

Figure 1-8: Window to edit material, alloy composition, and doping. ........................... 17

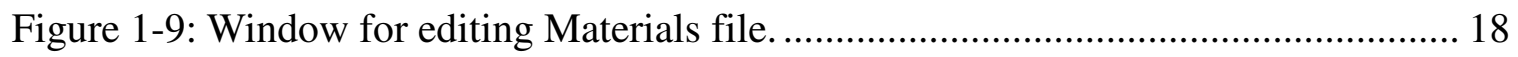

Figure 1-10: Editing Refractive Index and Bandgap energy in the Materials file ............ 18

Figure 1-11: GaN Laser Diode structure from Table 1-2 ............................................ 23

Figure 1-12: Zoomed in version of Laser Diode to focus on Quantum Well region

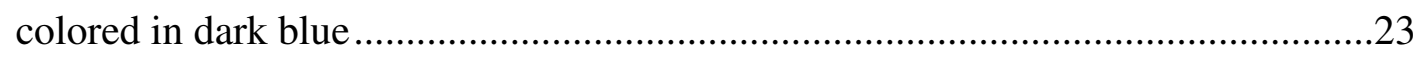

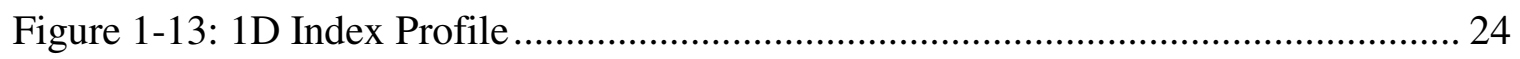

Figure 1-14: a) 1D Index Profile of Quantum wells b) Closer view of 1D Index

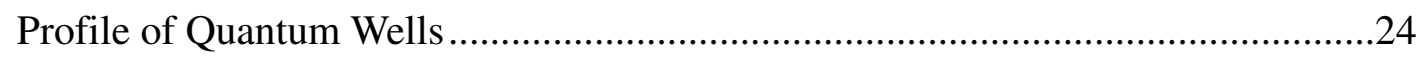


Figure 1-15: Optical Field (a.u.) vs. Y Distance from Sapphire Layer Axis for (a)

$0^{\text {th }}$ mode (b) $1^{\text {st }}$ mode (c) $2^{\text {nd }}$ mode (d) $3^{\text {rd }}$ mode (e) $4^{\text {th }}$ mode (f) $5^{\text {th }}$ mode $(\mathrm{g})$

$6^{\text {th }}$ mode- lasing (h) $7^{\text {th }}$ mode (i) $8^{\text {th }}$ mode $(j) 9^{\text {th }}$ mode .28

Figure 1-16: Mesh setup for a) original simulated file and b) new mesh setup

Figure 1-17: Optical Field (a.u.) vs. Y Distance from Sapphire Layer for (a) $0^{\text {th }}$ mode (b) $1^{\text {st }}$ mode (c) $2^{\text {nd }}$ mode (d) $3^{\text {rd }}$ mode (e) $4^{\text {th }}$ mode (f) $5^{\text {th }}$ mode $(\mathrm{g}) 6^{\text {th }}$ mode- lasing (h) $7^{\text {th }}$ mode (i) $8^{\text {th }}$ mode $(j) 9^{\text {th }}$ mode .31

Figure 2-1: Optical Field (a.u.) vs. Y Distance from Sapphire Layer for (a) $0^{\text {th }}$ mode (b) $1^{\text {st }}$ mode (c) $2^{\text {nd }}$ mode (d) $3^{\text {rd }}$ mode (e) $4^{\text {th }}$ mode (f) $5^{\text {th }}$ mode $(\mathrm{g}) 6^{\text {th }}$ mode-lasing (h) $7^{\text {th }}$ mode (i) $8^{\text {th }}$ mode $(j) 9^{\text {th }}$ mode 35

Figure 2-2: X and Y Direction of Model Laser Diode 36

Figure 2-3: OCF's Dependence on Mode and Y Distance from Sapphire Layer .............. 36

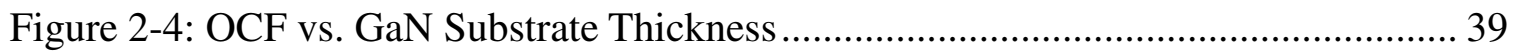

Figure 2-5: Optical Confinement Factor vs. Y Distance from Sapphire layer at Mode 6 for n-SL Thicknesses of a) $0.15 \mu \mathrm{m}$, and b) $0.8 \mu \mathrm{m}$ 43

Figure 2-6: Y-Axis shown in comparison to different layers 43

Figure 2-7: Optical Confinement vs. n-SL Thickness.............................................. 45

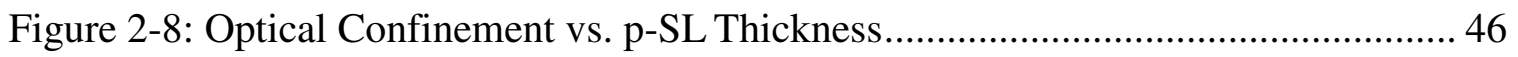

Figure 2-9: Y-Axis shown in comparison to different layers ..................................... 48

Figure 2-10: Optical Confinement vs. n-GaN Core Layer Thickness............................ 49

Figure 2-11: Optical Confinement vs. p-GaN Core Layer Thickness ............................ 51

Figure 2-12: Optical Confinement vs. p-GaN Contact Thickness ................................. 53 
Figure 3-1: 2D Plots of Optical Confinement vs. $\mathrm{X}$ and Y Distance for (a) $0^{\text {th }}$ mode (b) $1^{\text {st }}$ mode (c) $2^{\text {nd }}$ mode (d) $3^{\text {rd }}$ mode (e) $4^{\text {th }}$ mode (f) $5^{\text {th }}$ mode (g) $6^{\text {th }}$ mode- lasing (h) $7^{\text {th }}$ mode (i) $8^{\text {th }}$ mode (j) $9^{\text {th }}$ mode (k) $10^{\text {th }}$ mode (l) $11^{\text {th }}$ mode (m) $12^{\text {th }}$ mode (n) $13^{\text {th }}$ mode (o) $14^{\text {th }}$ mode (p) $15^{\text {th }}$ mode (q) $16^{\text {th }}$ mode- lasing (r) $17^{\text {th }}$ mode (s) $18^{\text {th }}$ mode (t) $19^{\text {th }}$ mode, and (u) $20^{\text {th }}$. .58

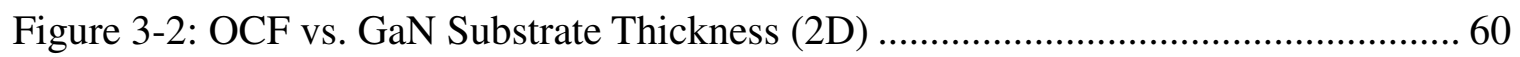

Figure 3-3: Optical Confinement vs. n-SL Thickness...................................................... 63

Figure 3-4: Optical Confinement vs. p-SL Thickness...................................................... 65

Figure 3-5: Optical Confinement vs. n-GaN Core Layer Thickness 2D ............................ 67

Figure 3-6: Optical Confinement vs. p-GaN Core Layer Thickness................................ 68

Figure 4-1: Optical Power vs. Current of the 1D Laser Diode .......................................... 72

Figure 4-2: Threshold Currents view of Optical Power vs. Current of 1D Laser Diode .. 73

Figure 4-3: Lasing Thresholds of Original Design and Optimized Design ...................... 76 


\section{CHAPTER 1: INTRODUCTION}

\subsection{Lasers Diodes, Light Emitting Diodes and Light}

In the visible color spectrum, there are three primary colors: red, green and blue, also referred to as RGB. Through the combination of these three colors, all the other colors in the spectrum can be created. For example, the combination of red and green would produce the color yellow. Current technology has found efficient ways at creating both red and green light using semiconductor technology, but the creation of blue light has not shared in this immediate success. Blue light has remained the hardest light to produce efficiently from semiconductor technologies. This has been the main road block preventing highly efficient laser diodes (LDs) from replacing our current lighting technologies.

Usages of light from semiconductor devices range among a variety of different applications. Traffic lights, outdoor megascreens, white lighting for homes, and optical disc reading are just a fraction of the technological advancements which blue light semiconductors have to offer. ${ }^{1}$ In the worldwide scheme of entertainment and consumer application, semiconductor lighting is at the apex of its eminent takeoff. "Now, if all the light bulbs in the World were replaced with these high efficiency laser diodes mass produced in visible wavelengths, the energy crisis would be over since it's been estimated that half the World's energy usage goes into lighting and most of this is presently highly inefficient. Incandescent lamps are only about 5 percent efficient; halogen lamps around 7 to 10 percent; and fluorescents, about 15 to 20 percent. High brightness LEDS suitable 
for lighting applications are advancing but are currently somewhere around halogen lamps in efficiency (though under some conditions, LEDs at low power may exceed 25 percent efficiency). But, it's unlikely that the LED could even match the laser diode due to the basic physics."

\begin{tabular}{|l|l|l|l|}
\hline \multicolumn{3}{|c|}{ Light bulbs } & \multicolumn{2}{c|}{ LEDs } \\
\hline \multicolumn{3}{|c|}{ Power consumption } \\
\hline Red: & 70 Watt & Red: & 18 Watt \\
\hline Yellow: & 70 Watt & Yellow: & 20 Watt \\
\hline Green: & 70 Watt & Blue-green: & 17 Watt \\
\hline Replacement interval & 5-10 years (estimated) \\
\hline $6 \ldots 12$ months \\
\hline Failure mode \\
\hline Sudden total failure & Gradual intensity decrease \\
\hline Visibility \\
\hline Uses color filter, reflects sunlight & Direct \\
\hline
\end{tabular}

Figure 1-1: Comparison of light bulbs and LEDs ${ }^{1}$

Blue violet light is close to the shortest wavelength of light in the visible spectrum. At a wavelength of $445 \mathrm{~nm}$, it still remains the hardest light to produce using semiconductors, because the bandgap properties of the materials needed to produce such a wavelength in semiconductors are hard to find. In recent years, many strides have been made in studying and researching Gallium Nitrate or Gallium Nitride $(\mathrm{GaN})$ as a material to yield blue light. The most progress and significant strides have been made by Dr. Nakamura, whom dedicated much of his research to the chemical growth of GaN compounds. Before Nakamura found a solution for growing GaN semiconductors, much of the focus for blue light semiconductors was spent on II-VI materials (where II and VI represent the group number on the Periodic Table of Elements). GaN is an III-V material 
and is much harder substance to deal with because of its higher lattice defects in comparison to II-VI materials. However with Nakamura's perilous efforts, he eventually found a technique to deposit $\mathrm{GaN}$ compounds and created the first $\mathrm{GaN}$ semiconductor. Since Nakamura has shown that GaN LED semiconductors are possible in 1995, fellow scientists across the world have worked hard to further the development and efficiency of blue light semiconductors.

\subsection{History of Blue Laser Diodes}

Laser diodes first became commercially viable during the 1980's at the dawn of compact disc (CD). Music was digitally "burned" into CDs and read using a small laser diode. The LDs emitted infrared (IR) light which is at the opposite end of the color spectrum from violet, at a wavelength of $780 \mathrm{~nm}$. CDs are still commonly used today to record music and data; however the storage allowed on a single $\mathrm{CD}$ is soon becoming obsolete in comparison to the optimum data storage a blue LD offers. During the mid 1990's, CD technology was replaced by DVD technology. Using the same concept, instead of the LDs emitting IR light like in CD technology, they now could emit red light at 635-650nm range. This resulted in an eight fold increase in overall storage capacity for DVD technology - increasing storage from $700 \mathrm{MB}$ to 4-5 GB! Latest technology also allowed for dual layers to be printed on a single disc thus doubling the eight fold increase to 16 times the space. The emergence of Blue-Ray discs that harnesses the technology of short wavelength light produces discs with a 50 GB capacity, thus continuing the trend in increased capacity, as LDs are created to emit shorter wavelengths. 
Laser pointers soon rose to popularity with their unique capability to point at objects or things on display without having to move a long pointing stick; just simply point and shoot. Unlike gas lasers that required cavities of $10 \mathrm{~cm}$ or greater to create the lasing action, smaller laser diodes could fit into pens and allowed for portable low powered devices emitting light in the $3-5 \mathrm{mw}$ range. ${ }^{2}$ There is not much interest in creating blue light laser pointers, but in any case, the technology is here.

So from a historical perspective, the significance of blue light LDs not only lies in the capability of producing efficient white light with the other main colors (red and green), but in the small wavelengths that allow for more data to be stored on discs.

During the breakthrough of blue-violet lasers, research was predominantly focused on II-VI compounds such as manganese-selenide, zinc-selenide, manganese-sulfide, etc. Groups such as 3M, SONY, Philips, and many universities achieved LEDs and lasers using II-VI materials, however they all had severe stability problems. Many of these devices would degrade within hours making any commercial delivery impossible. The instability of these II-VI based devices can be attributed to creation and propagation of defects within the lattice. III-V devices have better stability despite their inherent greater lattice mismatch. The lower defect density in II-VI devices still results in the creation and propagation of defects, while the III-V devices have more defects from the start, but because they're annealed at a higher temperature, they are more withstanding to the heat created by the lasing current action. 
The beginnings of blue-violet light research focused on II-VI devices due to two main problems in the creation of III-IV devices. The two major problems in creating III-V semiconductors were to produce sufficiently high quality crystalline layers and secondly to achieve p-type doping. The first problem of finding sufficiently high quality crystalline structures hasn't been completely solved, but sapphire has proven to be a stable substrate for which GaN can be grown on. The second problem of achieving p-type doping was solved by Akasaki of Nagoya University with the method of irradiation with low energy electrons (LEEBI). This enabled Akasaki to demonstrate the first GaN based blue LED in 1989. Using Akasaki's discovery as inspiration, Nakamura found his own way of doping the GaN using thermal annealing in a nitrogen atmosphere. ${ }^{1}$ Thus the foundations for further III-IV device research had been rooted and it was only a matter of time before efficient blue-violet LEDs would be commercially available.

This thesis focuses on the III-V materials instead of the II-VI thus following in Nakamura's research footsteps. This choice is mainly due to the ability of III-V materials to withstand the high temperatures without propagating defects. The beginnings of III-V laser diode devices were very elementary in their design compared to current designs. The diodes consisted of a substrate, cladding, core, and active region. Current designs have more complex structures such as a super lattice (SL), quantum wells and e-blocks. Using these new technologies devices with lower laser thresholds can be created thus leading to more efficient devices. 


\subsection{Differences Between LEDs and LDs}

LEDs and LDs differ in one main characteristic — the internal gain introduced by LDs. Laser Diodes have a mirror like cladding in the active region, which causes the internally produced photons to reflect back and forth inside this active region. The mirror like cladding region is known as the feedback cavity. The feedback cavity is nonexistent in LED devices.

The feedback cavity reflects the light back and forth within the cavity, resulting in stimulated emission. Stimulated emission is a process in which one photon causes the release of another photon. This process involves the first photon hitting an electron in its excited state and causing it to drop down to ground state thereby releasing a photon with equal frequency, direction and phase as the first photon. The emitted waves that result from the stimulated emission are coherent, in that all the waves are of the same wavelength and share the same phase. Since all the waves are of the same wavelength, the emitted spectrum width (the wavelengths of light emitted) is much narrower than that of an LED. Without the feedback cavity LEDs mainly emit light through spontaneous emission, while on the other hand LDs emit light through stimulated emission. If the injection current is high enough, the LD will begin lasing. ${ }^{3}$

\subsection{Laser Diode Structure: Further Into How an LD Works}

\subsubsection{Cladding and Core Layers}

Early in LD development, the LD structure was based on the p-n junction diode. This is still the case today. It is important to remember that unlike a normal p-n junction 
diode, in LDs there exists an active layer that is sandwiched between cladding layers (the $\mathrm{p}$ and $\mathrm{n}$ is the bread as shown in heterostructure of Figure 1-2). This active layer is the optical feedback cavity and it is where the photons are reflected back and forth until they are able to escape as coherent light.

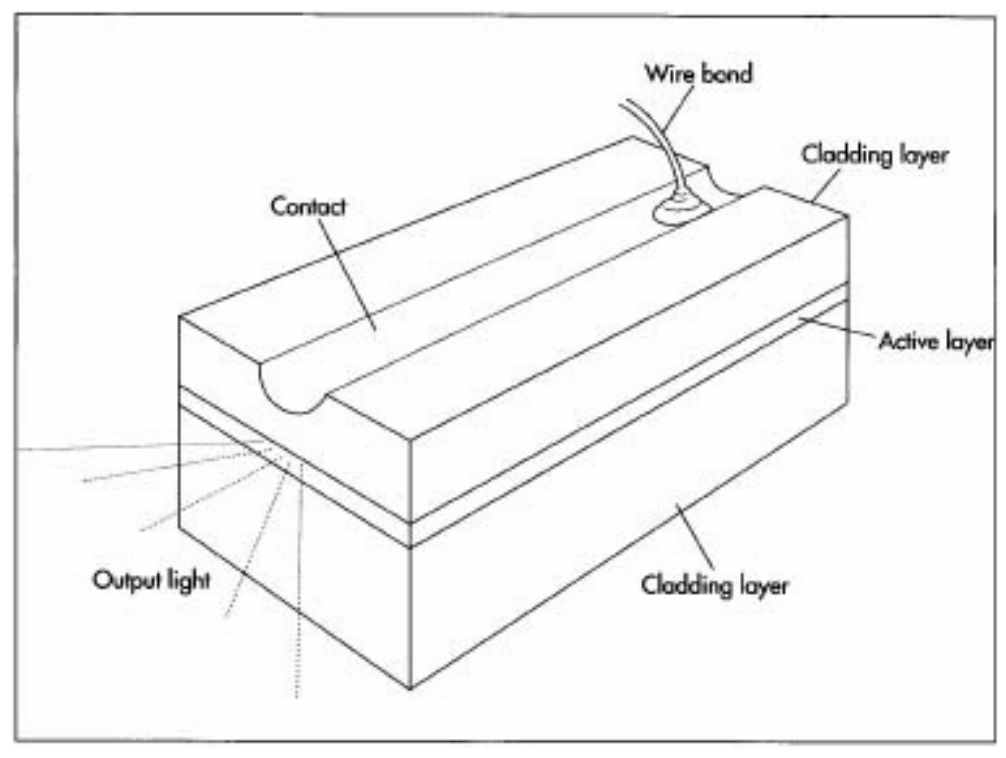

Figure 1-2: Single Heterostructure Laser

The active layer has a larger refractive index than the cladding layers, so in accordance with Snell's Law the light will be confined in the active region. The active layer is usually surrounded by a thin layer of a material (core layer) that has a refractive index on the order of the active layer. The core and cladding layers combine to create a waveguide that confines the light within the active region of the LD. Similar to the optical properties of a fiber optic cable, the light is confined in between the cladding layers staying within the core and active layers.

The super lattice (SL) is a material that has very thin alternating layers of two materials. The purpose of a SL is to take advantage of the tunneling properties associated 
with crystal lattice systems, while still maintaining control over energy bandgap design. By selecting only a single material, one is automatically limiting themselves to the crystal structure properties of that material. By selecting a SL, the user has the opportunity to create an alloy material that has user-defined properties associated with the combination of the two materials. SLs are similar to quantum wells (QW) in that both share a periodic well type energy bandgap diagram as shown in Figure 1-3, but the main difference in SL structures is the electrons are able to tunnel through the thinner walls. Quantum wells are designed so that tunneling is not possible and thus the carriers become very dense inside the quantum well. When the density builds up in the quantum well, the only exit is to combine with a hole in the valence band and thus emit light in the form of spontaneous emission. In SL structures, this phenomenon does not occur, but instead the electrons are able to escape the wells and tunnel across the material. By using SL structures, the user can control the movement of the carriers, the bandgap of the material, and the refractive index of the material surrounding the active layer. The SL structure provides the designer with a variety of techniques for tweaking the properties of the LD.

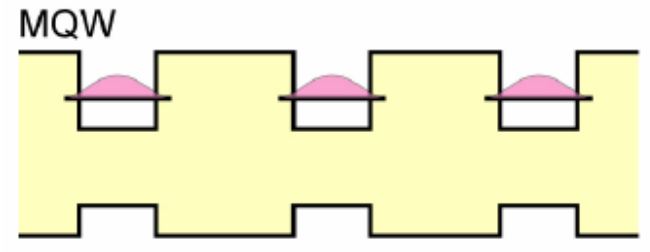

(a)

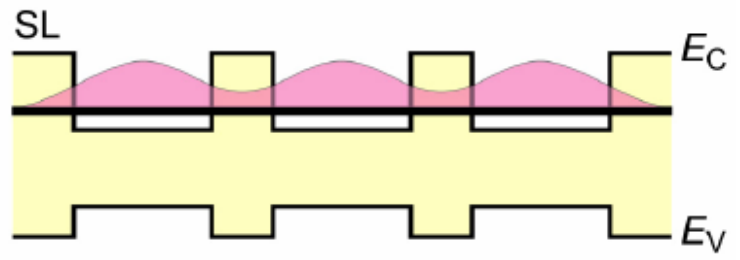

(b)

Figure 1-3: (a) Multiple Quantum Well and (b) Super-Lattice structures. 


\subsubsection{Substrate and Buffer Layer}

The buffer is a thick deposit of an undoped material that is used to help match the lattice constants of the oxide layer and the cladding layer. It is deposited between these two layers to reduce the number of defects. In III-V LDs, a GaN buffer layer (several microns in thickness) is deposited on the oxide substrate prior to growth of the actual device. This buffer layer can become a waveguide — trapping light within the thick substrate layer, because it is surrounded by two layers that have a lower refractive index. Having another waveguide besides the active region waveguide is detrimental to the function of the laser because it traps light away from the lasing region. However, the buffer layer is necessary to match the crystal lattices of the AlGaN and sapphire layers. The GaN buffer layer fulfills its function of lowering lattice defects, but its waveguidelike properties also lead to the occurrence of ghost modes (modes that do not contribute to lasing and instead steal energy away from the lasing action). Since the buffer layer is so thick, the effective refractive index can change over its thickness producing different transverse ghost modes. The active region is very thin and has a constant effective refractive index which only allows one optical mode to exist compared to the buffer layer. ${ }^{4}$ Thus the buffer layer can contain a variety of modes, while the active region will only contain one mode that occurs at the lasing mode.

\subsubsection{Quantum Wells $(Q W)$ Layer}

Quantum wells are designed to trap electrons in a 2-D environment. The wells are designed to have a particular bandgap energy related to the wavelength of light emitted by 
the laser diode. The quantum well allows the electrons to gather more densely in the well than they would elsewhere. It is easy to think of it as though the well is drawing electrons towards it then trapping them. (In fact it isn't so much that the wells are drawing electrons to them and away from the other parts of the semiconductor; instead electrons keep falling into the well, and the well becomes saturated and full that it seems as though the well is attracting electrons.) This packs more electrons in the active region and allows more electrons to jump the bandgap thereby releasing a photon. The size of the well must be on order of the wavelength of light hoping to be produced. Each well is created by creating a thin layer (the well) and surrounding it by thicker layers of a different material (bulk). Thus for a multi quantum well (MQW) design, there would be alternating layers of bulk material and quantum wells. The well layer is made from the normal active layer material with a lower refractive index then the surrounding bulk layers. This in a sense is like having many tiny optical cavities that interact with photons at the quantum level. It can be shown that the more quantum wells in a semiconductor, the larger the threshold current becomes. This is because when there are more wells for the electrons to fall into, a higher current is necessary to provide the quantum wells with enough electrons to maintain saturation which in turn is the foundation of lasing. ${ }^{5}$

Quantum wells have many advantages in the design a laser diode structure. First, by using QWs, one has the freedom to design the transition energies, which ultimately determines the wavelength of light that leaves the active region in the form of spontaneous emission. Second, since QWs have a small volume, the free carrier concentration in the QW is high and at high free carrier concentrations non-radiative deep-level transitions are less likely thus yielding a high radiative efficiency. Third, due to 
the small size of the quantum wells, the carrier density required to achieve population inversion (i.e., high carrier density in the conduction band, compared with the valence band) is small and therefore the threshold current density of QW structures is low. And finally, surface recombinations are less likely making surface recombination less important in the study of QWs. ${ }^{5}$ These advantages are summed up in Figure 1-4.

\begin{tabular}{|l|}
\hline Advantages of QW structures: \\
\hline Freedom to design transition energies. \\
\hline High radiative efficiency. \\
\hline Low laser thresholds. \\
\hline Surface recombination. \\
\hline
\end{tabular}

Figure 1-4: Summary of advantages by implementing QW structures.

\subsubsection{Electron Block (e-Block) Layer}

The e-Block is a p-doped material that has a larger bandgap than its neighboring materials. The e-Block is a very thin layer that is added next to the active layer to prevent electrons from leaking into the p-doped side. Electrons that overflow into the p-type side leads to leakage current. Leakage current is detrimental to the operation of the LD because the higher current causes heating and dissipates non-lasing energy creating an inefficient LD. In Figure 1-5b the doped structure contains an AlGaN blocking player that prevents electrons in the active region from moving into the p-type side. The e-block only blocks electrons and allows the holes to move freely from the p-type side into the active region unaffected. If a high current is injected across the diode, a large carrier 
concentration results in the active region. This leads to enhanced non-radiative carrier recombination at defects and to an escalation of electron leakage from the quantum wells into the p-side of the diode, despite the AlGaN blocker layer. ${ }^{6}$ Thus the e-block is very useful in preventing leakage current up to a certain current, but once the injected current becomes very high, the blocking properties are null and the leakage electrons causes the laser to heat, degrade, and eventually breakdown. Increasing the bandgap of the e-block can be an effective method of making it very hard for electrons to leak onto the p-side. However, an increase in the bandgap of the e-block often means a higher content of Al and therefore a decrease in the thermal and electrical conductivities. The decrease in the thermal and electrical conductivities counteracts the improvement of the large bandgap eblock $^{7}$.

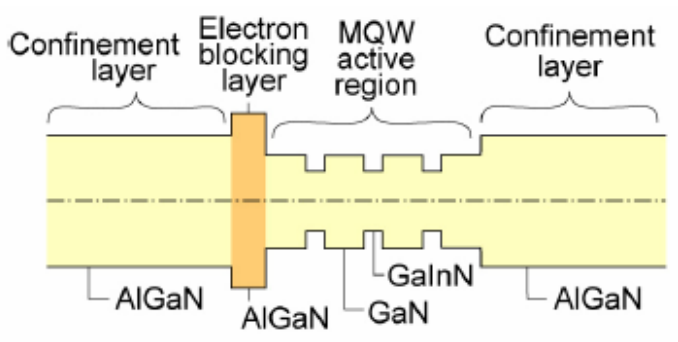

(a)

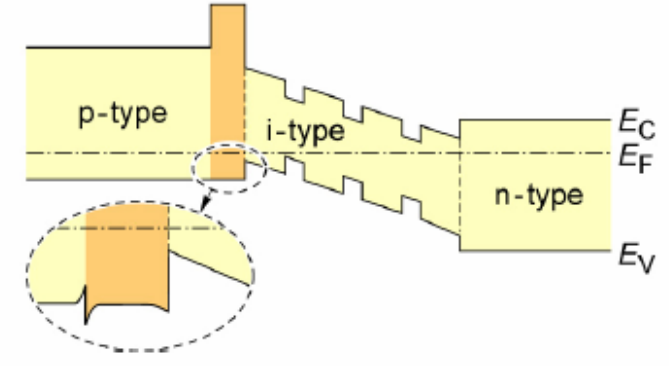

(b)

Figure 1-5: AlGaN current-blocking layer in an AlGaN/GaN/GaInN MQW LED structure.

(a) Band diagram without doping. (b) Band diagram with doping. The Al content in the electron-blocking layer is higher than in the p-type confinement layer. 


\subsection{RSOFT Software - LaserMOD}

\subsubsection{Introduction to LaserMOD}

LaserMOD is an integrated software package developed by RSOFT for the design and simulation of semiconductor lasers and active photonic structures. LaserMOD is a fully integrated platform with a user friendly parametric CAD interface, nonuniform Delauney mesh generator, material libraries, gain and mode calculation utilities, simulation engine, standard and custom plot generation utilities, and versatile graphical viewing utilities. ${ }^{8}$ In short the LaserMOD program provides the user with an immense environment in which semiconductor devices can be modeled and tested based on their user specified properties.

\subsubsection{Mathematical Model - 8x8 Kronig-Penney (KP) Model}

The LaserMOD simulation software uses the Kronig-Penney (KP) model to model the bandgap relationships of the different materials. The KP model is based upon the splitting of allowed electron energies as the interatomic distance between atoms decreases to form a crystal. The KP method involves quantum mechanics and a solution to Schrödinger's wave equation. ${ }^{3}$ For the one dimensional crystal structure, a periodic well function can be used to represent the crystal lattice structure. This is because when the potential functions of atoms are brought close together, the net potential function of the overlapping regions is similar to a periodic function. Using the specified characteristics of a periodic function for the boundary conditions for Schrödinger's equation, a plot of the energy $\mathrm{E}$ as a function of wave number $\mathrm{k}$ can be generated, which describes the valence 
and conduction bands and the allowed energy bands. However for two dimensional calculations, matrices become a necessary addition to efficiently solve Schrödinger's wave equation. A popular method for solving E vs. $\mathrm{k}$ diagrams is using $8 \times 8$ matrices. Using special matrix rules and "tricks" (i.e. Helmholtz equation) these calculations can be solved with a computer program. Many programs have been written that can solve these types of mathematical problems-I chose to use RSOFT's LaserMOD. Rather than set each mathematical problem up based upon the different properties of the materials in the semiconductor and input them into a math program such as MatLab, RSOFT created a program that has a CAD interface which can easily and quickly calculate all the necessary properties of semiconductors for the user.

\subsubsection{Optical Confinement Factor (OCF)}

The optical confinement factor (OCF) of a chosen mode is defined as the ratio of the total guided energy of all the modes to the energy of the chosen mode located in the active region. ${ }^{9}$ A higher $\mathrm{OCF}$ for a certain mode indicates there is more energy in the active region for that mode. High energy in the active region is usually a sign that lasing

is occurring. Thus, whichever mode has the highest OCF will be the mode at which lasing occurs.

The optical modes are determined from the solution of the Helmholtz equation via simultaneous iteration then LaserMOD is able to calculate the OCF for each mode.

$$
\left(\frac{\partial^{2}}{\partial x^{2}}+\frac{\delta^{2}}{\delta y^{2}}+k_{0}\left(\varepsilon(x, y)-n_{e f f, m}^{2}\right)\right) E_{m}(x, y, z)=0
$$




$$
\text { Where } E_{m}(x, y, z)=E_{m}(x, y) \exp \left(i k_{0} n_{e f f, m} z\right)
$$

Where $\mathrm{k}_{0}$ is the free-space wave vector, $\varepsilon(x, y)$ is the complex dielectric constant profile of the multiplayer structure. The eigenvalues are given by the effective index $n_{\text {eff,m }}$. The frequency $k_{0}=\omega_{0} / c$ for which the mode is solved can be specified in LaserMOD. The frequency is set to correspond to the quantum well band gap energy. ${ }^{8}$ The OCF can be thought of as the fraction of energy of mode that is located at the active region. ${ }^{10}$

\subsubsection{Program Modification}

The first step is to draw the semiconductor laser diode using the CAD tools in

LaserMOD.

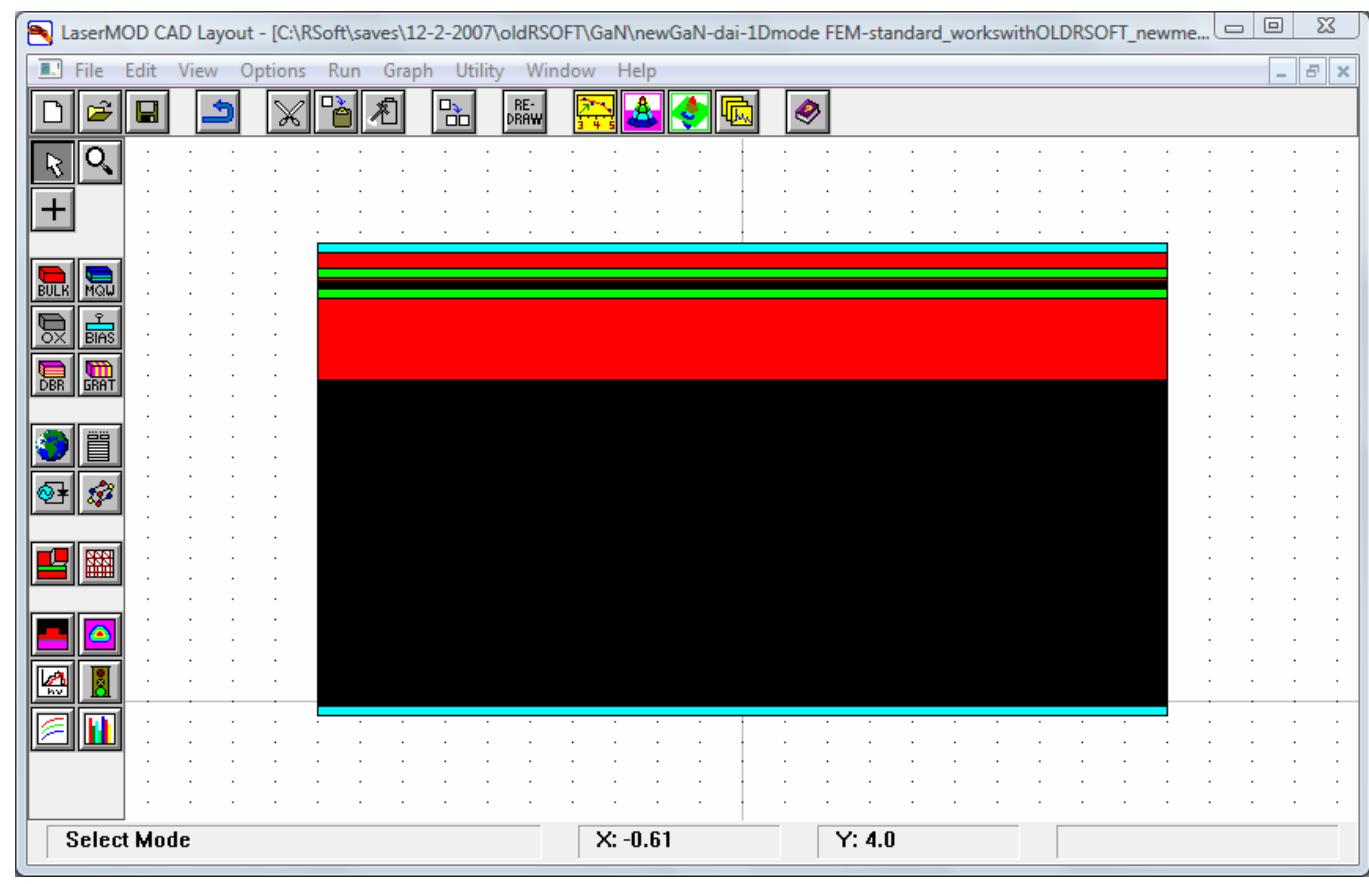

Figure 1-6: RSOFT LaserMOD software. A GaN Laser Diode is currently drawn. 
LaserMOD has a variety of materials that one can choose from when creating a semiconductor. One can right click on the material that has been drawn and change its characteristics such as the type of material, thickness, width, doping levels, alloy percentages, etc.

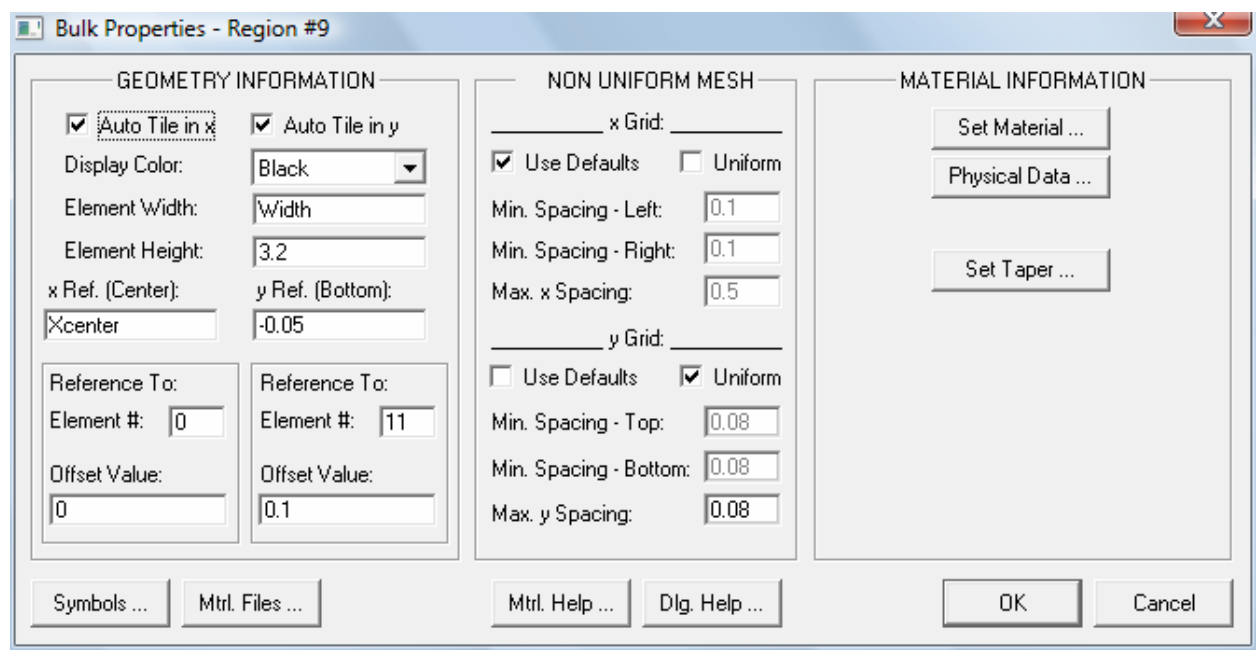

Figure 1-7: Window to edit individual bulk layer 


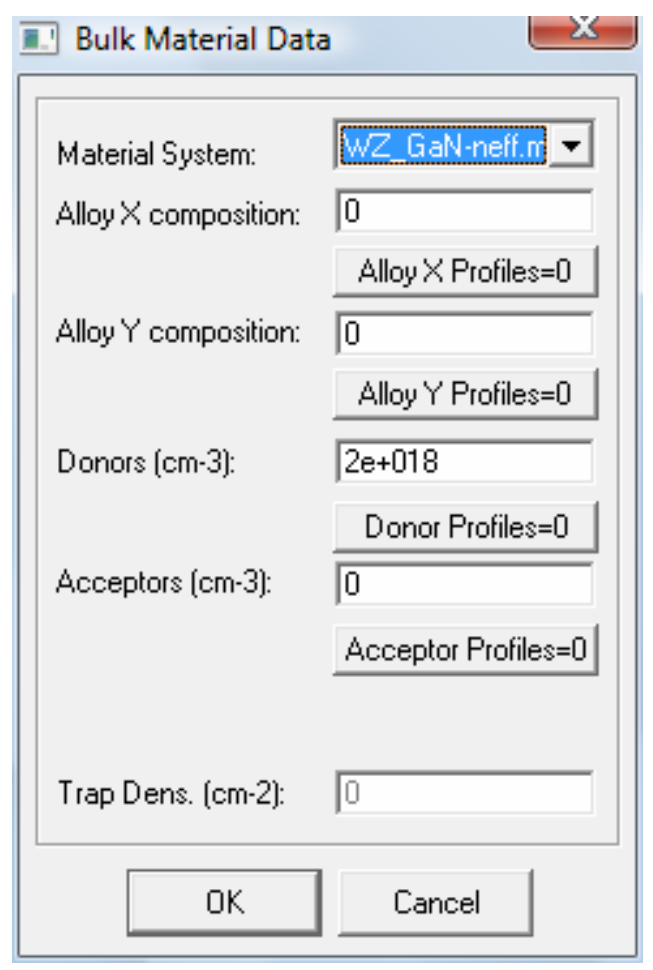

Figure 1-8: Window to edit material, alloy composition, and doping.

After changing these materials to make a working semiconductor, many simulations can be run. There are refractive index simulations, bandgaps simulations, optical simulations, current vs. voltage simulations, etc. For a list of additional LaserMOD simulations please refer to the LaserMOD User Guide. ${ }^{8}$

The first step to getting applicable 1-D simulations was to edit the materials file (Figure 1-9) and customize the properties of the materials used. Thus the refractive indexes of certain chemical compounds were altered to represent actual materials. The material and its respective refractive index are shown in Table 1-1. 
Table 1-1: Materials of the LD and corresponding refractive indices

\begin{tabular}{|c|c|}
\hline Material & Refractive Index (n) \\
\hline $\mathrm{GaN}$ & 2.55 \\
\hline $\mathrm{AlGaN}$ & 2.5 \\
\hline $\mathrm{AlN}$ & 2.1677 \\
\hline $\mathrm{InN}$ & 3.6836 \\
\hline $\mathrm{InGaN}$ & 2.75 \\
\hline $\mathrm{Al}_{2} \mathrm{O}_{3}$ & 1.77 \\
\hline
\end{tabular}

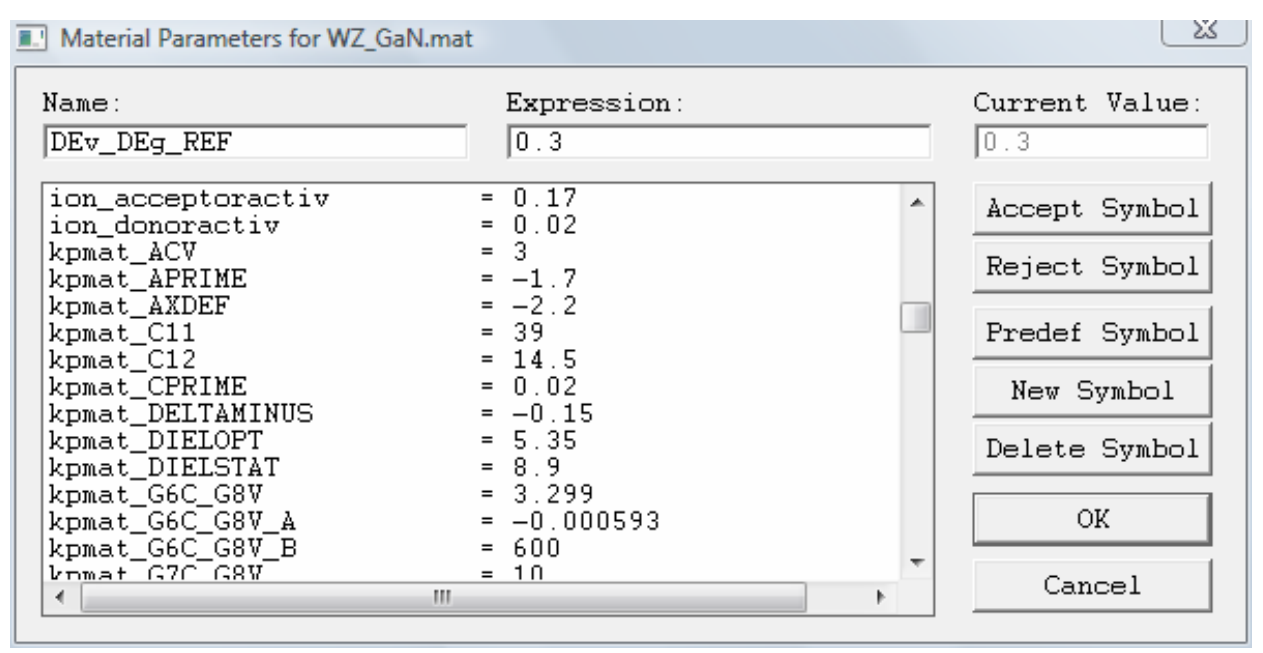

Figure 1-9: Window for editing Materials file.

$\begin{array}{cc}\text { kpmat_DIELOPT } & =6.5025 \\ \text { wz_Eg } & =3.3\end{array}$

Figure 1-10: Editing Refractive Index and Bandgap energy in the Materials file 
The refractive index is inputted as its value squared. So when editing the materials file for GaN, the kpmat_DIELOPT was changed to $2.55^{2}$, which is 6.5025 .

When creating the alloys $\mathrm{AlGaN}$ and $\mathrm{InGaN}$ it is possible to add different percents of GaN. It is common notation to specify the percents of each material using subscripts such as this, $\mathrm{Al}_{0.14} \mathrm{Ga}_{0.86} \mathrm{~N}$ and $\mathrm{In}_{0.15} \mathrm{Ga}_{0.85} \mathrm{~N}$. The first number is considered the $x$ percent, and the second number is the $1-x$ percent, thus in the case above the AlGaN material is $14 \% \mathrm{AlN}$ and $86 \% \mathrm{GaN}$ and likewise the $\mathrm{InGaN}$ material is $15 \% \mathrm{InN}$ and $85 \% \mathrm{GaN}$.

After creating an alloy, it is necessary to calculate the refractive index of that alloy. This can be done with the following algebraic equation:

$\mathrm{n}_{\mathrm{AlGaN}}=\mathrm{n}_{\mathrm{AIN}} \mathrm{X}+\mathrm{n}_{\mathrm{GaN}}(1-\mathrm{x})$

where $\mathrm{n}$ is the refractive index of the material and $\mathrm{x}$ is the percent of material

This is the same method that Nakamura employed for calculating the refractive indices of his SL structures. ${ }^{7}$ In our case the refractive index of all the materials are listed in Table 1-1, except AlN and InN. Thus the equation above is used to solve the missing refractive indexes of AlN and InN. The materials file is edited with the resolved refractive indexes of AlN and InN.

It is important to carefully choose what percent of each compound will be used in the alloy, because changing the percent of each material affects the bandgap of the overall alloy material as well as its lattice constants. Changing the percent of each compound affects the lattice matching with the materials above and below it. For blue light lasing, the perfect amount of each compound in the alloy InGaN will result in good lattice matching (fewer defects) and a bandgap that yields high efficiency blue light. 
Another change in the program that needed to be made is incorporating the super lattice (SL) structure into the LaserMOD software. The LaserMOD software doesn't have a SL material option, so to recreate a SL structure; each individual alternating layer would have to be manually drawn. Another option instead of manually drawing each layer would be to use a bulk layer that represents the bulk properties of the SL material. Thus to model the SL layer, a bulk layer with the equivalent refractive index of the $\mathrm{InGaN}$ structure was used. Using a bulk layer to model the SL structure saves the user from having to draw each alternating layer and simplifies the amount of calculations over the space of the layer. It would require a tremendous amount of processing power to calculate the relationships between each alternating layer of the SL structure. 


\subsubsection{Building in LaserMOD}

The LD structure that is modeled is shown in Table 1-2. This LD structure was designed at Peking University in China. This design is a separate confinement heterostructure $(\mathrm{SCH})$ and includes an oxide, substrate, cladding, quantum well, e-block, cladding, and contact layers. An SCH is defined as a structure having an active layer, core layers and cladding layers. Most commercial laser diodes use an SCH.

After drawing all the rectangular layers (as shown in Figure 1-11 and Figure 1-12), the next step is to attach each layer to the layer above and below it and properly add in the alloy and doping concentrations to each layer. According to the Peking University specifications, all the $\mathrm{n}$-doping was doped at $2 \times 10^{18} \mathrm{~cm}^{-3}$ and all the $\mathrm{p}$-doping was done at $1 \times 10^{17} \mathrm{~cm}^{-3}$. Next, electrodes at the top and bottom of the diode are added. The addition of the electrodes concluded the modeling process, permitting the LD structure to be properly simulated. 
Table 1-2: Laser diode layer structure and parameters

\begin{tabular}{|c|c|c|}
\hline Layer & Thickness (nm) & Refractive Index (n) \\
\hline $\begin{array}{l}\text { p-GaN } \\
\text { (contact) }\end{array}$ & 50 & 2.55 \\
\hline $\begin{array}{c}\text { p- } \mathrm{Al}_{0.12} \mathrm{Ga}_{0.88} \mathrm{~N} / \mathrm{GaN} \\
\text { (p-SL cladding) }\end{array}$ & 500 & 2.53 \\
\hline $\begin{array}{l}\mathrm{p}-\mathrm{GaN} \\
\text { (p-core) }\end{array}$ & 100 & 2.55 \\
\hline $\begin{array}{c}\mathrm{p}-\mathrm{Al}_{0.35} \mathrm{GA}_{0.65} \mathrm{~N} \\
\text { (e-block) }\end{array}$ & 20 & 2.42 \\
\hline n-GaN & 15 & 2.55 \\
\hline $\begin{array}{c}\mathrm{In}_{0.1} \mathrm{GA}_{0.9} \mathrm{~N} / \mathrm{GaN} \\
(5 \mathrm{QWs})\end{array}$ & 67 & $2.685 / 2.55$ \\
\hline $\begin{array}{l}\text { n-GaN } \\
\text { (n-core) }\end{array}$ & 100 & 2.55 \\
\hline $\begin{array}{c}\mathrm{n}-\mathrm{Al}_{0.12} \mathrm{Ga}_{0.88} \mathrm{~N} / \mathrm{GaN} \\
(\mathrm{n}-\mathrm{SL} \text { cladding })\end{array}$ & 800 & 2.53 \\
\hline $\begin{array}{c}\mathrm{n}-\mathrm{GaN} \\
\text { (Substrate or buffer) }\end{array}$ & 4000 & 2.55 \\
\hline $\begin{array}{l}\text { Sapphire } \\
\text { (Oxide) }\end{array}$ & 4000 & 1.77 \\
\hline
\end{tabular}




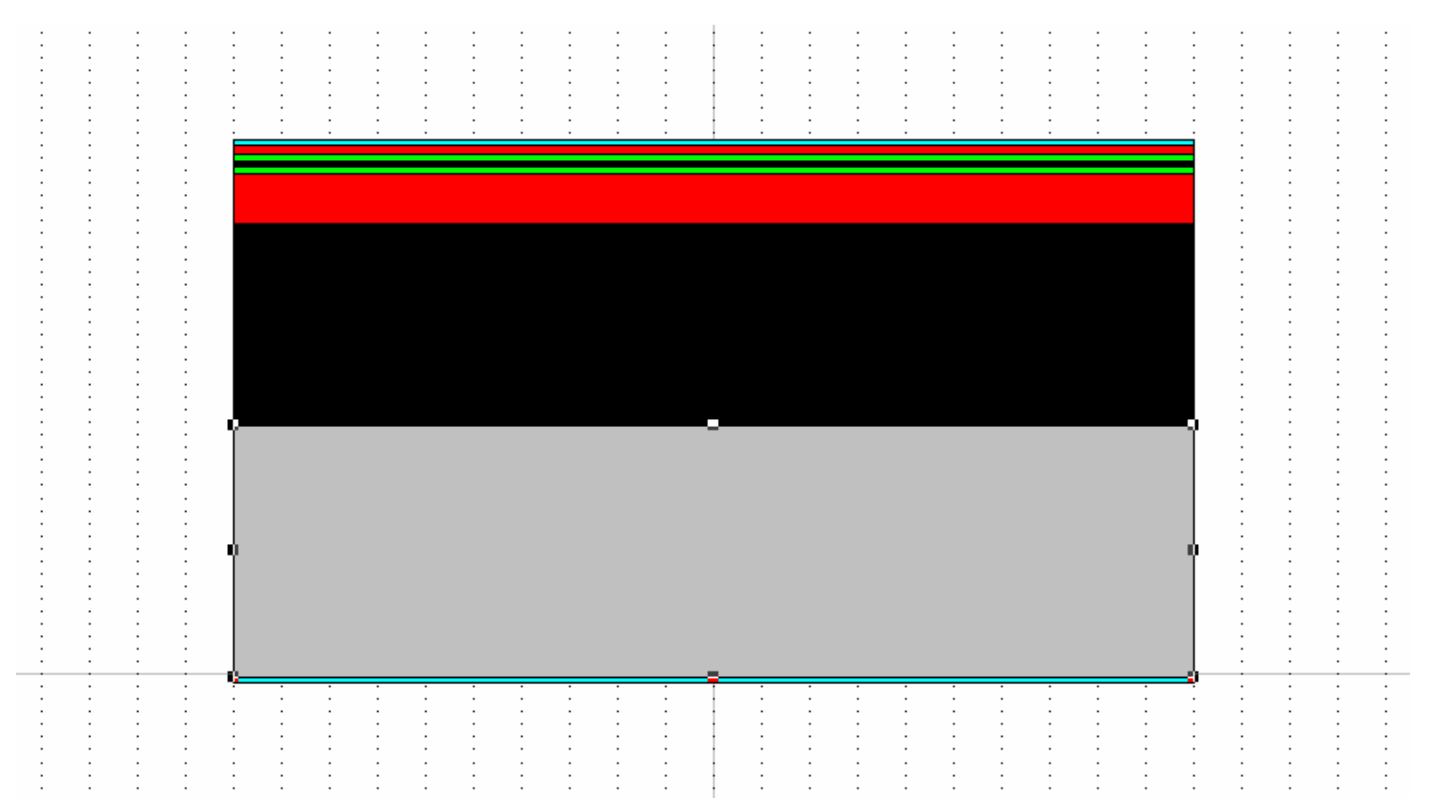

Figure 1-11: GaN Laser Diode structure from Table 1-2
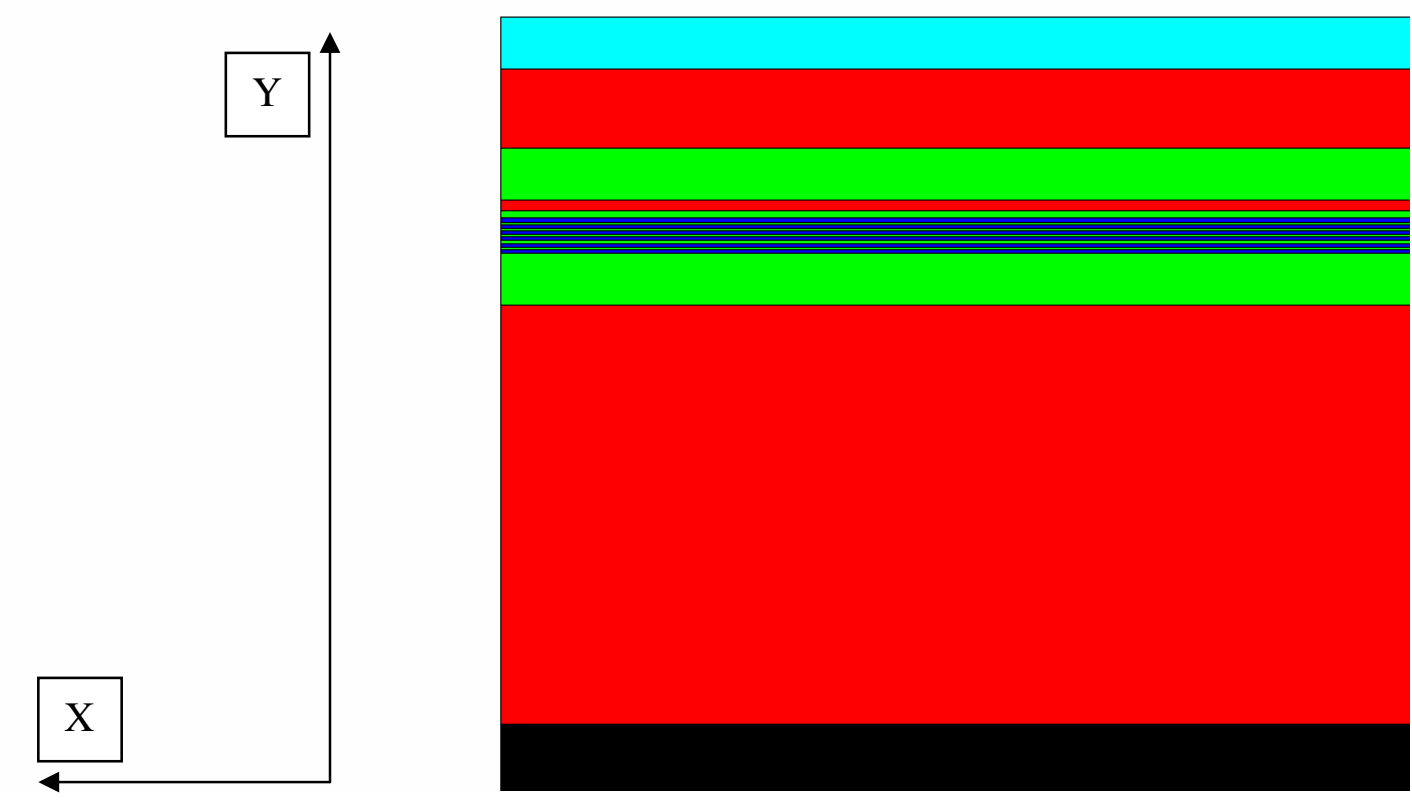

Figure 1-12: Zoomed in version of Laser Diode to focus on Quantum Well region colored in dark blue 
The first simulation run is the index profile simulation. This simulation graphs refractive index vs. the vertical y position of the laser structure. This simulation provides a visual way to verify that the indexes of refraction are correct.

Refractive Index Profile (at $x=-0.5$ )

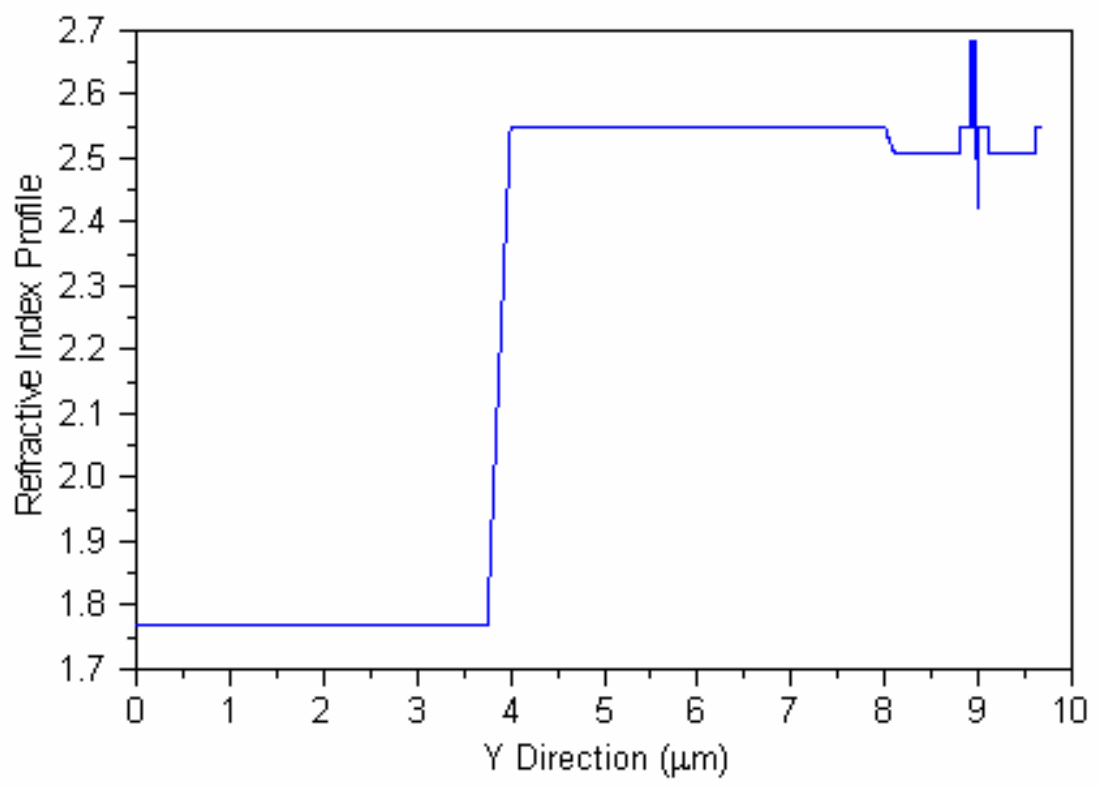

Figure 1-13: 1D Index Profile

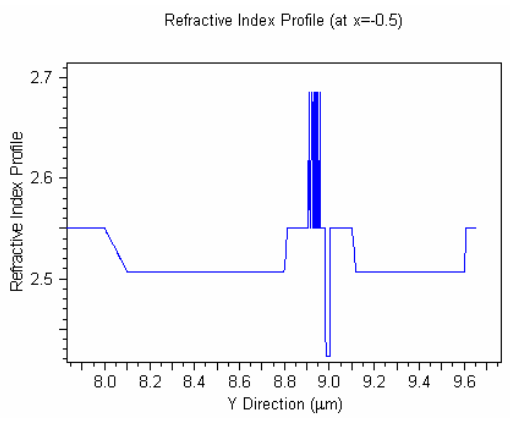

(a)

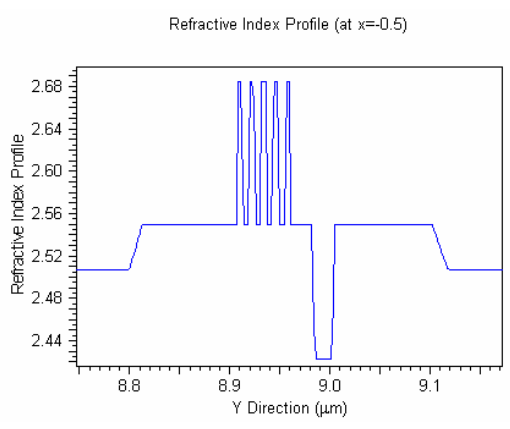

(b)

Figure 1-14: a) 1D Index Profile of Quantum wells b) Closer view of 1D Index Profile of Quantum Wells 
It is clear from Figure 1-13 and Figure 1-14 that each layer's respective refractive index is taken into account. Moving from left to right corresponds to moving from the bottom of the diode design to the top. Thus moving from the bottom of the diode up, the layers have respective refractive indices of $1.77,2.55,2.53,2.55$, then alternating between 2.685 and 2.55 for the quantum wells, $2.55,2.42,2.55,2.53$, and finally ending at 2.55. All the layers are completely accounted for and thus the transverse coupled waveguide is in place. It is called a waveguide because of the way the refractive indices are set up around the active region and at the substrate. It is called a coupled waveguide because the two waveguides (one located at the active region and the other located at the substrate layer) physically interact with each other when the LD is biased.

\subsubsection{LaserMOD Simulation Setup}

The simulation is run for the first 10 transverse modes, i.e., modes 0-9. It is found that the lasing mode was the $5^{\mathrm{h}}$ mode because it has the highest optical field (a. u.) out of all the other modes and it also has the greatest overlap with the quantum well structure. The lasing mode shows only one maximum value of optical field located at the position of the active region. All other modes have many occurrences of minima and maxima values of optical field. These minima and maxima are related to the mode number that the light is oscillating at. From waveguide theory it is expected that the higher the mode number (any mode except for the fundamental) the more minima and maxima will exist. Our first simulation is run with errors and is shown in Figure 1-15. 
The peaks of the different modes in Figure 1-15 seem to appear in no reoccurring pattern and do not agree with waveguide theory. The fundamental mode and the $1^{\text {st }}$ order mode have patterns to what is expected in an oscillating waveguide, but after the $1^{\text {st }}$ mode, the waveguide no longer shows increased oscillations as the mode order is increased.

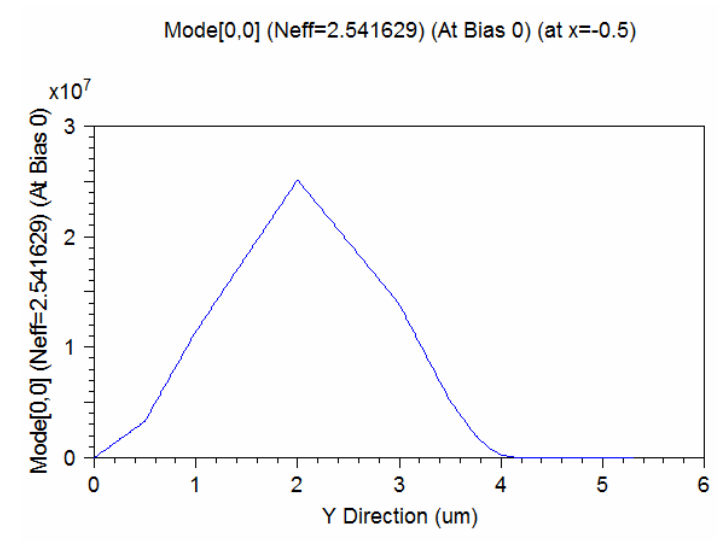

(a)

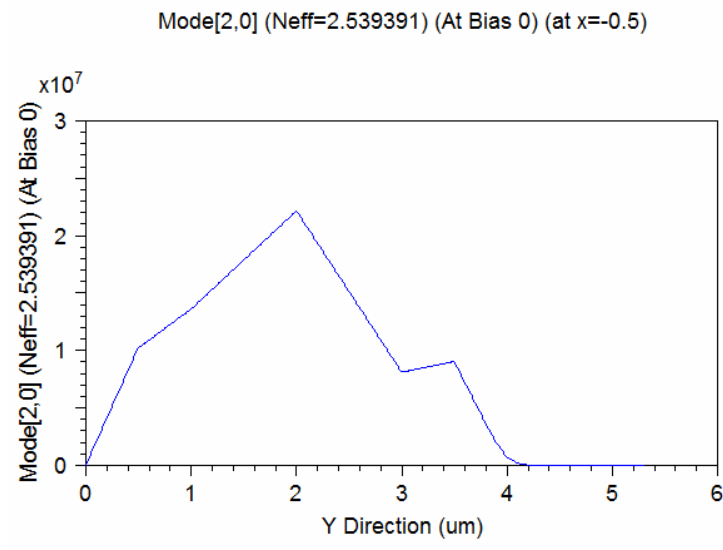

(c)

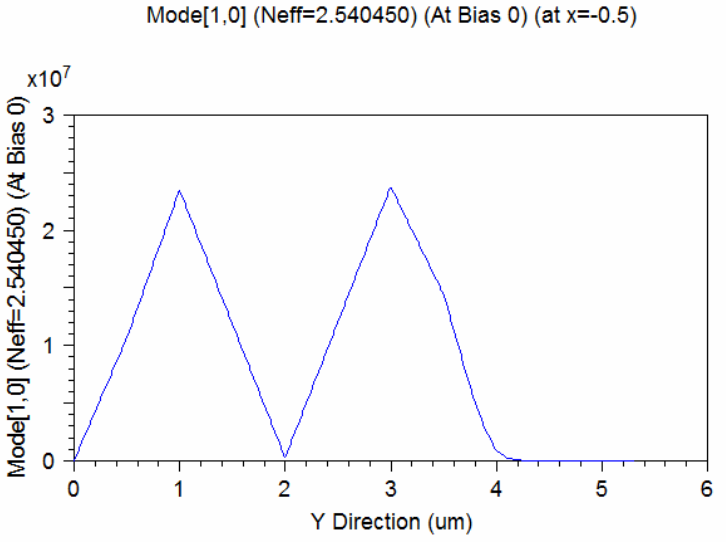

(b)

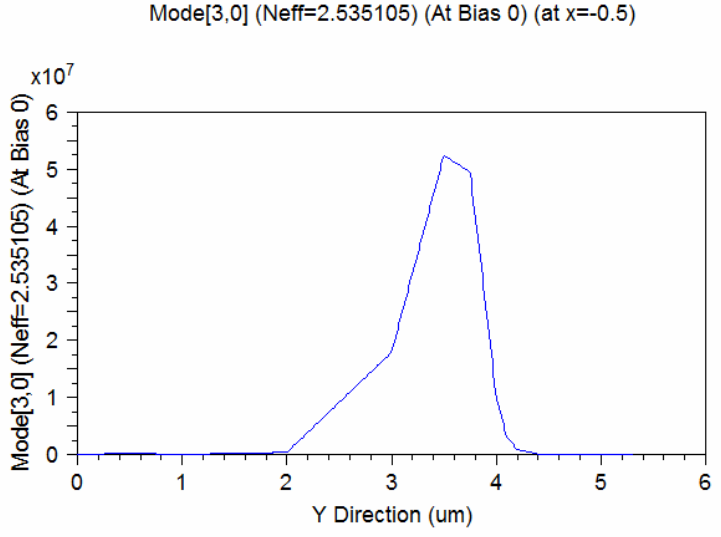

(d) 
Mode[4,0] $($ Neff $=2.534211)($ At Bias 0$)($ at $x=-0.5)$

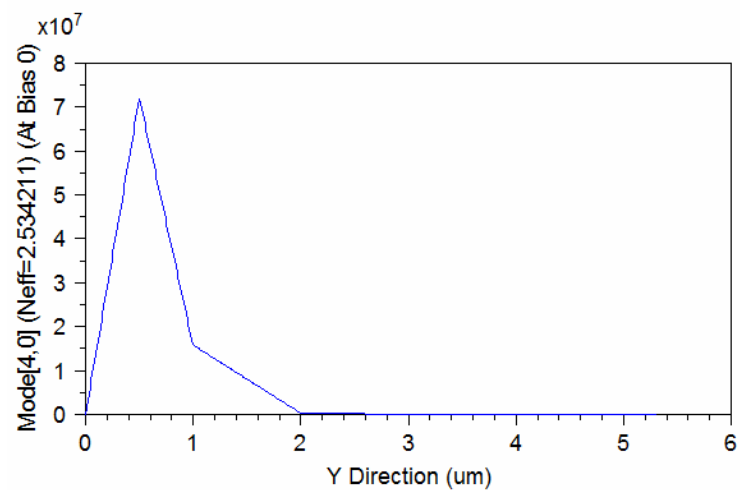

(e)

Mode $[6,0]($ Neff $=2.521786)($ At Bias 0) $($ at $x=-0.5)$

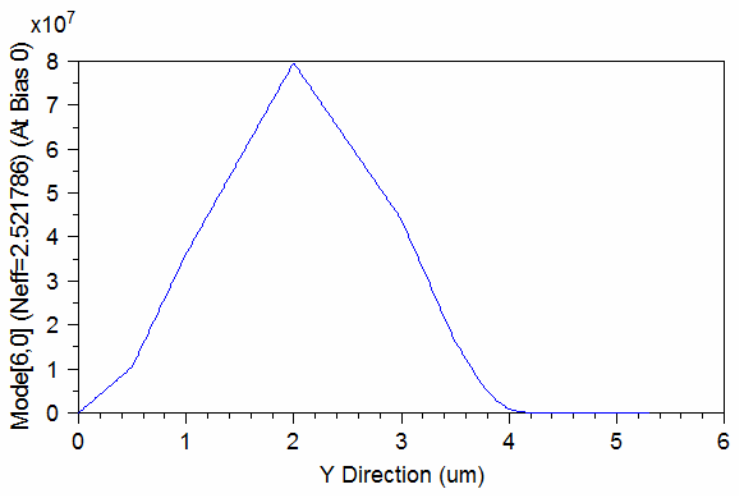

(g)
Mode $[5.0]($ Neff $=2.526194)($ At Bias 0) $($ at $x=-0.5)$

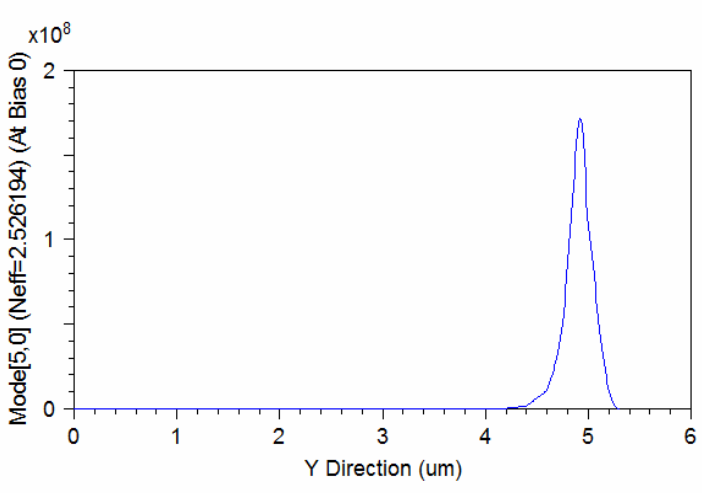

(f)

Mode $[7,0]($ Neff $=2.520598)($ At Bias 0$)($ at $x=-0.5)$

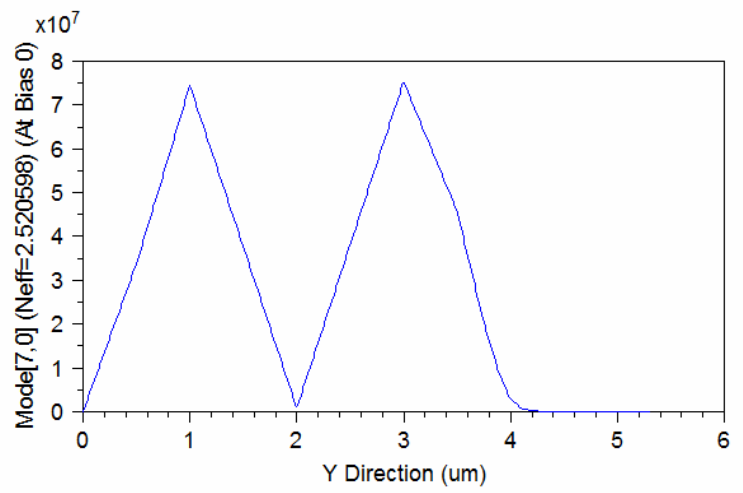

(h) 


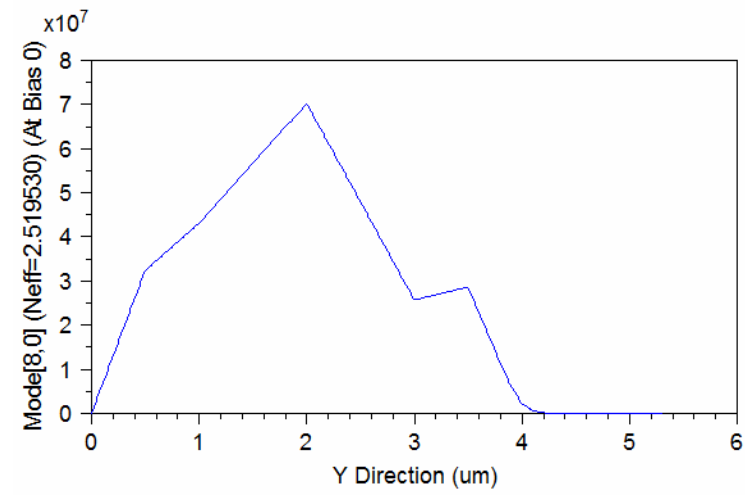

(i)

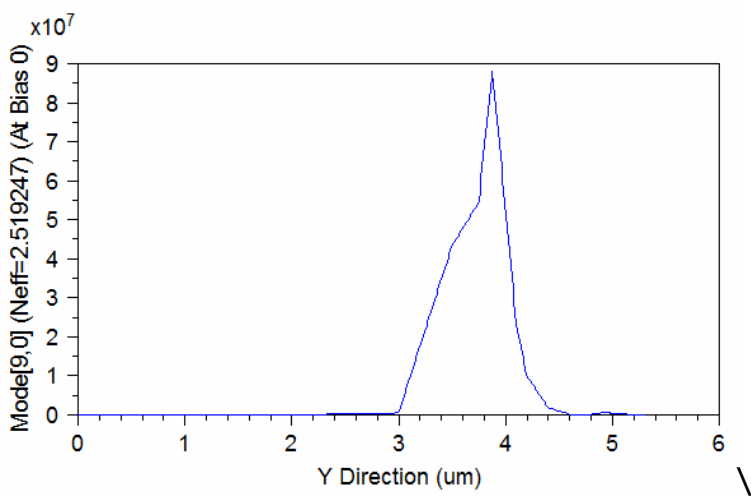

$(j)$

Figure 1-15: Optical Field (a.u.) vs. Y Distance from Sapphire Layer Axis for (a) $0^{\text {th }}$ mode (b) $1^{\text {st }}$ mode (c) $2^{\text {nd }}$ mode (d) $3^{\text {rd }}$ mode (e) $4^{\text {th }}$ mode (f) $5^{\text {th }}$ mode $(\mathrm{g}) 6^{\text {th }}$ mode- lasing (h) $7^{\text {th }}$ mode (i) $8^{\text {th }}$ mode $(j) 9^{\text {th }}$ mode

In LaserMOD, there is a mesh system that breaks the laser system into chunks, and then LaserMOD runs the physics model over these chunks. Since the GaN substrate is so large and is not of primary interest for lasing, the LaserMOD program automatically sets up a mesh for this layer with very large chunks, to make the simulation run faster. Thus the errors in Figure 1-15 arise from the simulation not being run in detail over the GaN substrate. The solution was to decrease the distance between each mesh point so that the simulation is run much more in depth over the GaN substrate layer. From Figure 1-16 it is apparent that the original file had a very largely spaced mesh in the GaN substrate area, and the new simulation file uses a much finer mesh. 


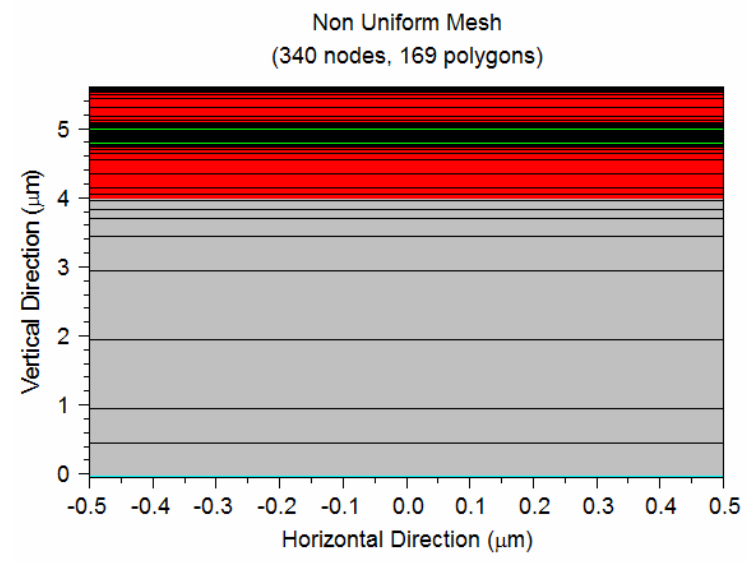

(a)

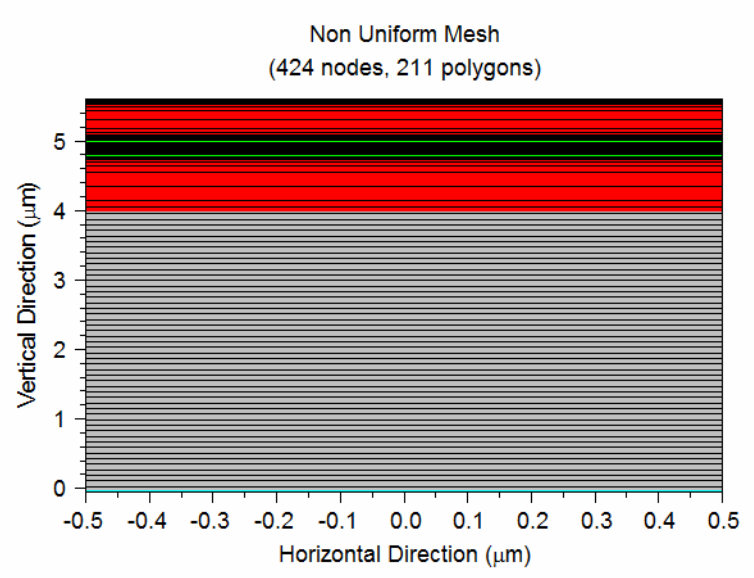

(b)

Figure 1-16: Mesh setup for a) original simulated file and b) new mesh setup

The results for our model with a finer mesh are much more accurate and in line with waveguide theory and are shown in Figure 1-17. Each mode is clearly shown oscillating and it can now be seen how the optical field intensity of each of these modes compare to one another. As the mode order is increased, the number of oscillations increases. This relationship will be further evaluated in the next chapter. 


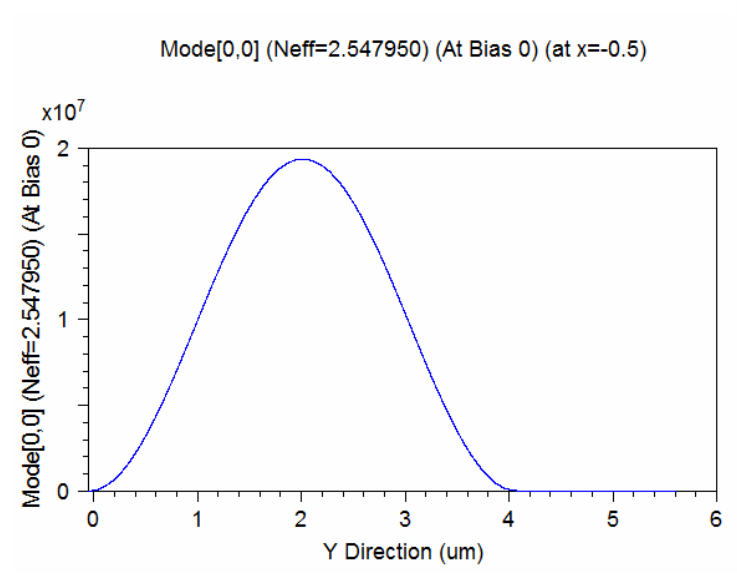

(a)

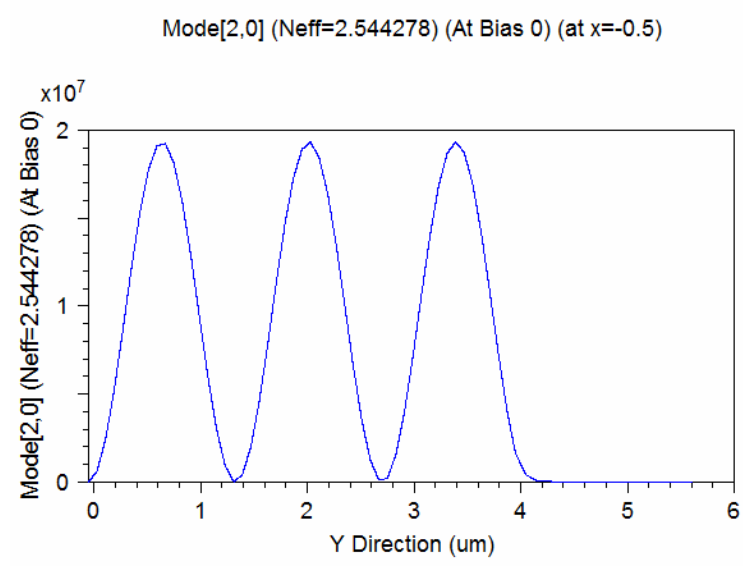

(c)

Mode[4,0] $($ Neff $=2.537000)($ At Bias 0$)($ at $x=-0.5)$

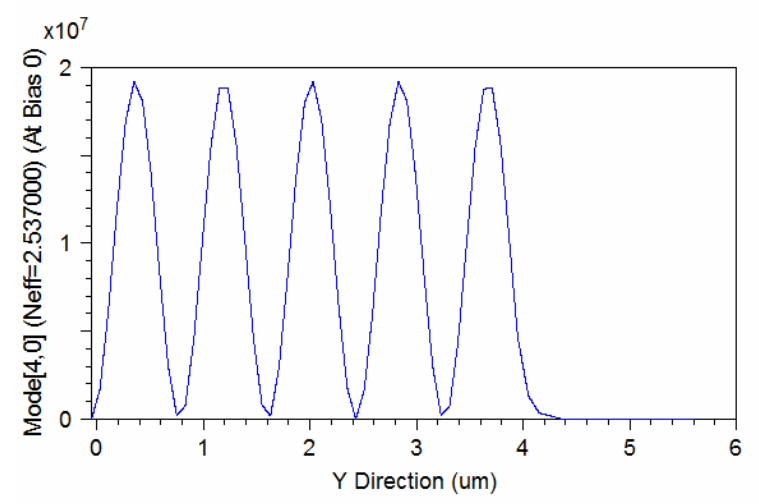

(e)

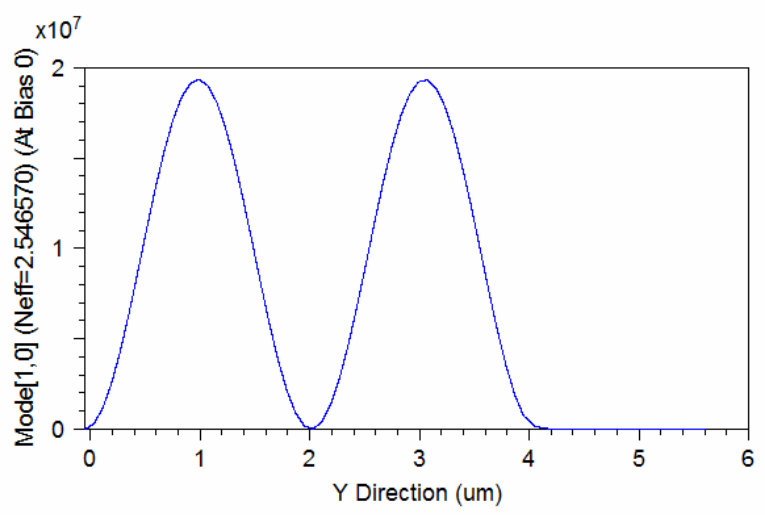

(b)

Mode $[3,0]($ Neff $=2.541082)($ At Bias 0$)($ at $x=-0.5)$

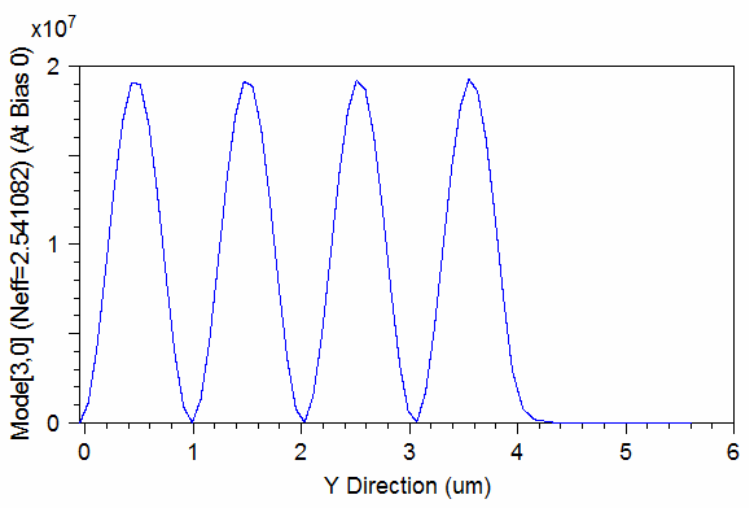

(d)

Mode $[5,0]($ Neff $=2.532056)($ At Bias 0$)($ at $x=-0.5)$

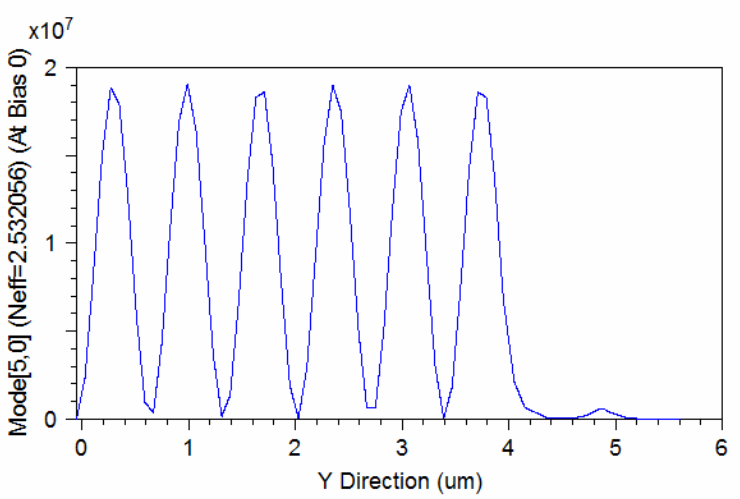




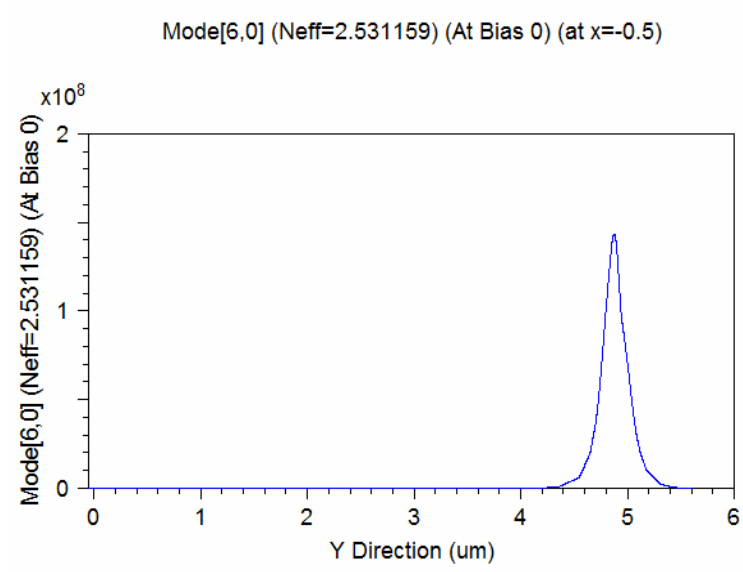

(g)

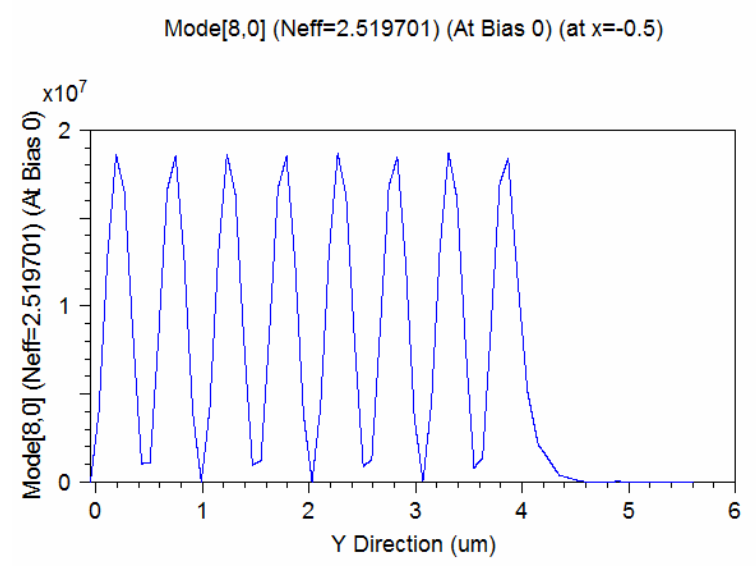

(i)

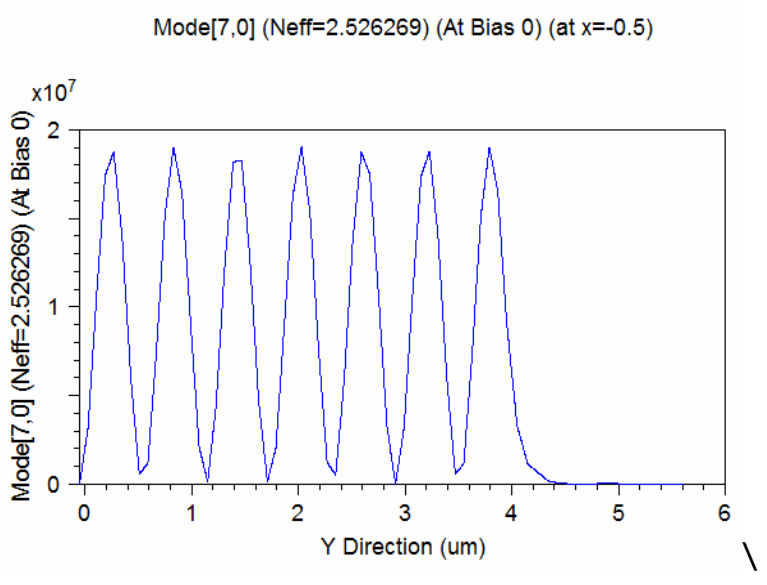

(h)

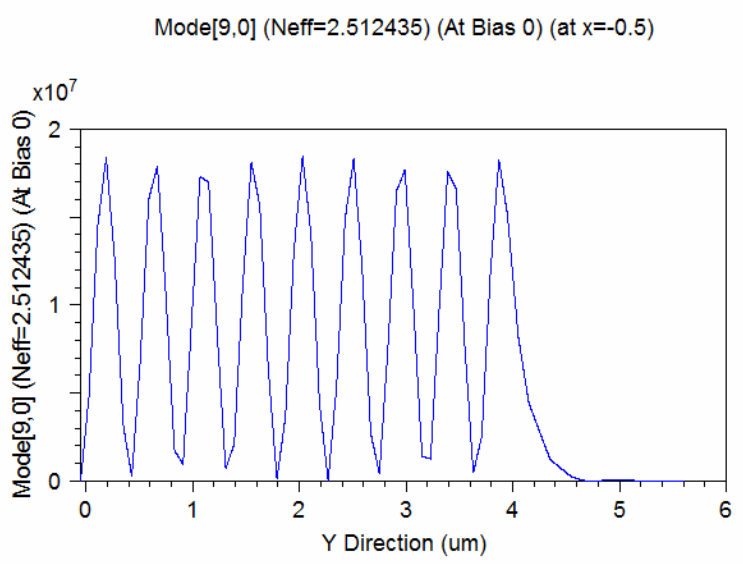

(j)

Figure 1-17: Optical Field (a.u.) vs. Y Distance from Sapphire Layer for (a) $0^{\text {th }}$ mode (b) $1^{\text {st }}$ mode (c) $2^{\text {nd }}$ mode (d) $3^{\text {rd }}$ mode (e) $4^{\text {th }}$ mode (f) $5^{\text {th }}$ mode (g) $6^{\text {th }}$ mode- lasing (h) $7^{\text {th }}$ mode (i) $8^{\text {th }}$ mode (j) $9^{\text {th }}$ mode 


\section{CHAPTER 2: OPTICAL MODE 1D SIMULATIONS}

\subsection{Optical Modes in 1D Simulations of GaN LDs}

There are two types of waves in a waveguide-longitudinal and transverse waves. Longitudinal waves are waves that have oscillations and transmit energy in the direction of propagation. Transverse waves are waves that oscillate and transmit energy perpendicular to the direction of propagation. When waves are trapped in a waveguide, the waves bounce back and forth off the boundaries of waveguide causing the waves to overlap and interact in a constructive and deconstructive manor. The parts of the wave that interact in a deconstructive manor cancel out, and those that interact constructively form modes. Thus longitudinal and transverse modes are formed within the waveguide. Cavity Surface Emitting Lasers (VCSELs) emit light based upon transverse modes, while Distributed-feedback (DFB) and Fabry-Perot (FP) harness the properties of longitudinal modes. When studying GaN LDs it is important to study the radiation emitted by the transverse interaction of the electromagnetic waves.

The structure of a GaN LD is made up of a core, cladding, active region, substrate, and buffer layers. When these layers are combined to form a functional LD, the combination brings rise to a larger waveguide that effects the lasing of the diode. We discussed earlier that when creating a waveguide, a material of high refractive index is surrounded by a layer of lower refractive index. When all the independent layers of the LD are combined, it ends up creating a very large waveguide in which the substrate acts as a core layer and the cladding and oxide layers that surrounds it acts as cladding layers. Thus light that escapes the active region ends up in the substrate layer and is then guided 
between the cladding and oxide layers. Since the active region is so thin, it only can support one optical mode. However, the waveguide that is formed in the substrate layer is very large and thus can support a larger number of optical modes. Therefore it is important to recognize that the combination of all the different layers in a GaN LD produces lasing that takes place at a mode above the fundamental mode.

Many laser designs operate in the fundamental mode, but due to the unique substrate waveguide structure of GaN LDs, GaN LDs can operate at higher order modes. ${ }^{4}$, ${ }^{7,11,12,13}$ Since nitride lasers have cladding refractive indices that are similar to the GaN contact layers (p-contact and n-substrate), modal energy can often times leak out of the waveguide and into the GaN contact layers—-preventing the fundamental mode from being the lasing mode. Therefore since the laser does not always oscillate in the fundamental mode but instead in a higher order mode, all the modes that do not contribute to the lasing are known as ghost modes or anti-guide modes. These modes are competing with and steal energy away from the lasing mode. It is of interest to make these ghost modes less effective by lowering their contribution or OCF while maximizing the lasing mode's OCF. 


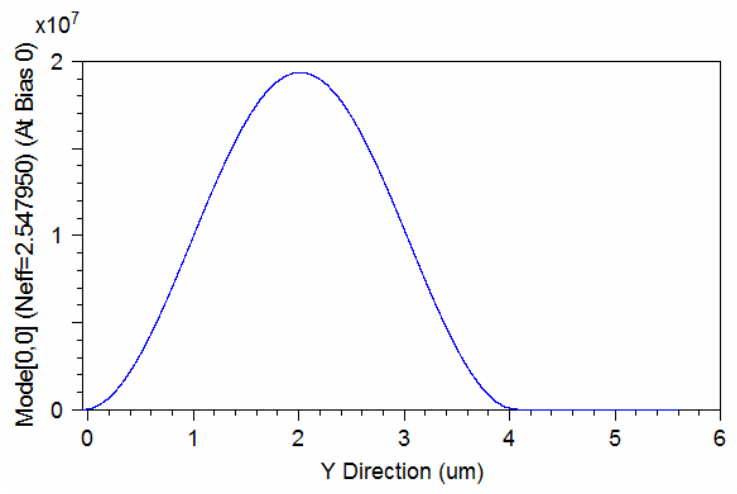

(a)

Mode $[2,0]($ Neff $=2.544278)($ At Bias 0$)($ at $x=-0.5)$

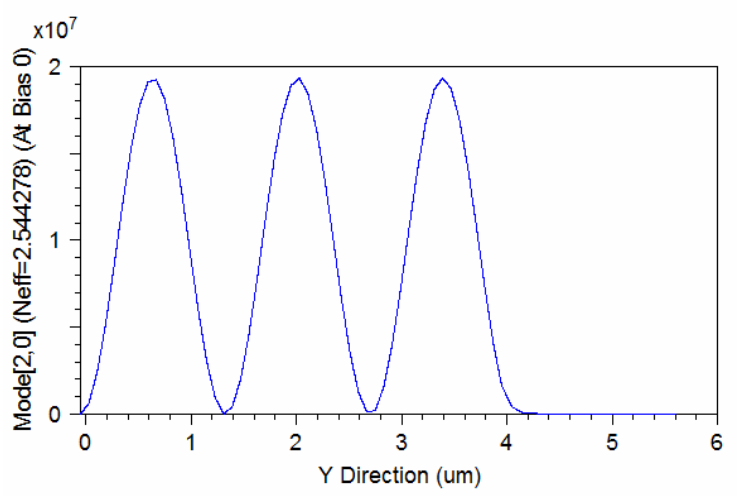

(c)

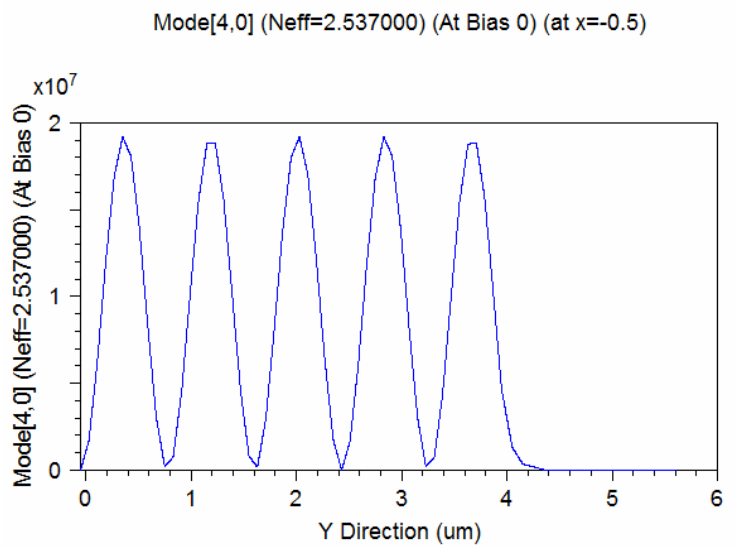

(e)

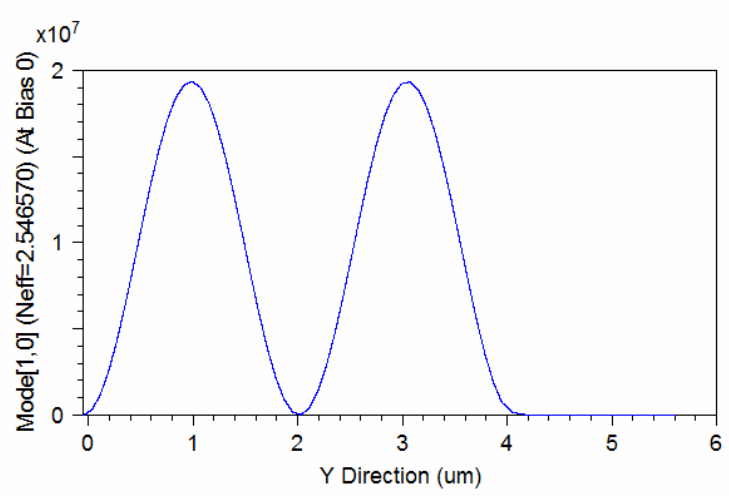

(b)

Mode $[3,0]($ Neff $=2.541082)($ At Bias 0$)($ at $x=-0.5)$

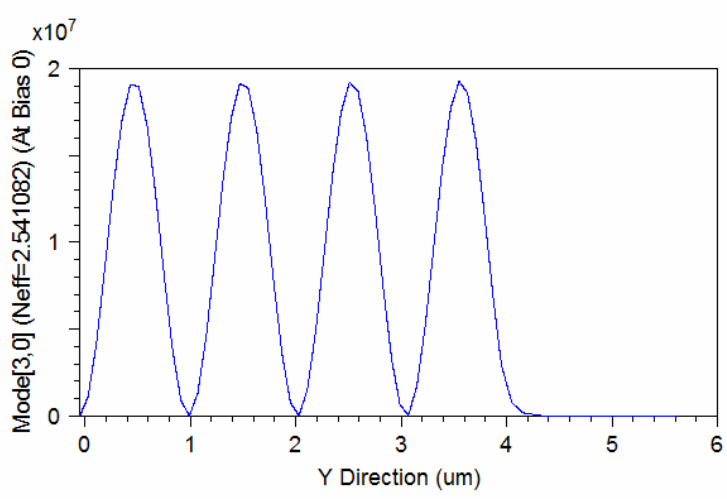

(d)

Mode[5,0] $($ Neff $=2.532056)($ At Bias 0$)($ at $x=-0.5)$

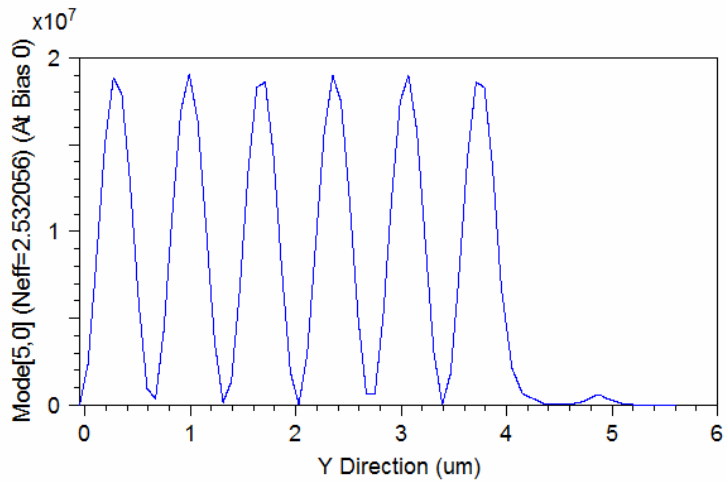

(f) 


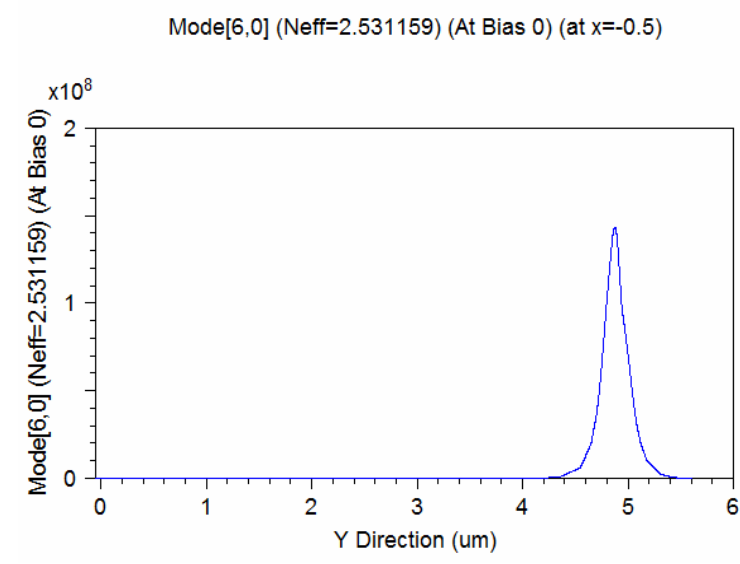

$(g)$

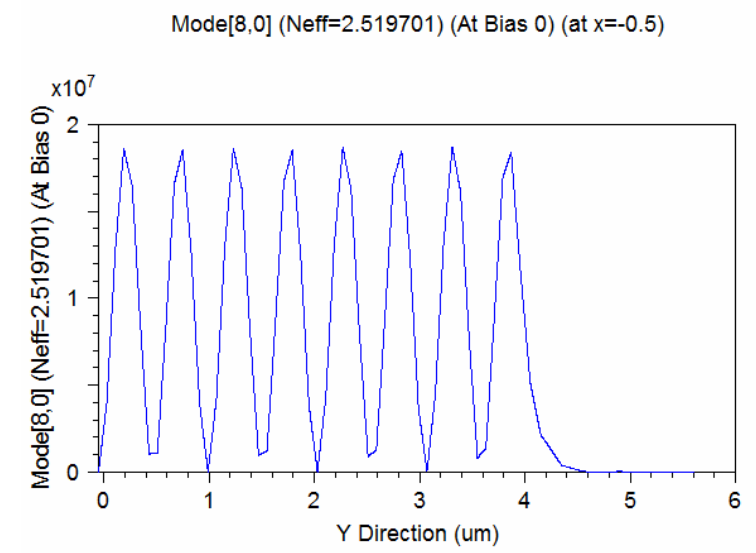

(i)

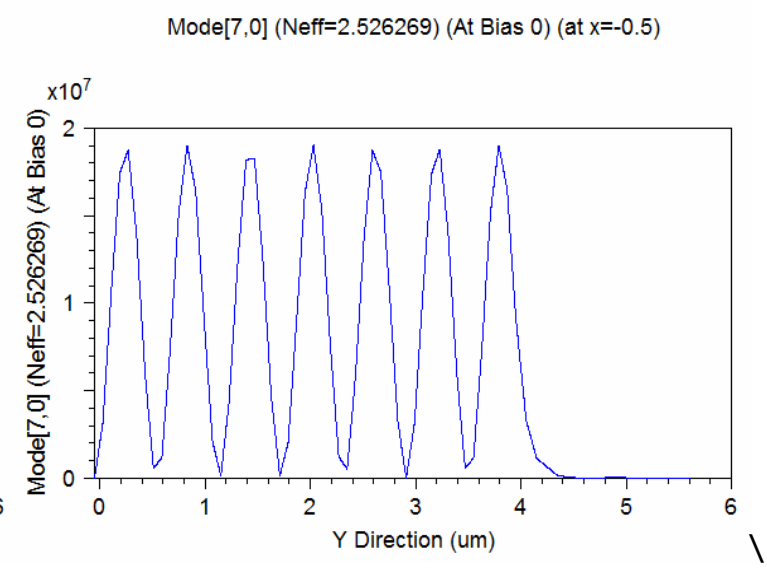

(h)

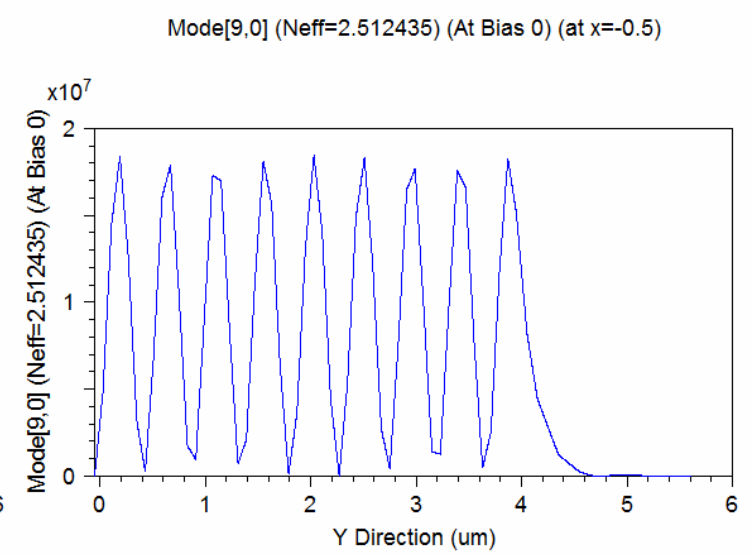

$(j)$

Figure 2-1: Optical Field (a.u.) vs. Y Distance from Sapphire Layer for (a) $0^{\text {th }}$ mode (b) $1^{\text {st }}$ mode (c) $2^{\text {nd }}$ mode (d) $3^{\text {rd }}$ mode (e) $4^{\text {th }}$ mode (f) $5^{\text {th }}$ mode (g) $6^{\text {th }}$ mode-lasing (h) $7^{\text {th }}$ mode (i) $8^{\text {th }}$ mode (j) $9^{\text {th }}$ mode

Figure 2-1 shows the first 10 transverse modes of our GaN LD. As the mode number is increased, the number of oscillations increases. However, for the $6^{\text {th }}$ mode there is only one oscillation and it is centered over the active region. Thus the $6^{\text {th }}$ mode is the lasing mode and has an optical intensity much greater than the optical intensity of the other transverse modes. 


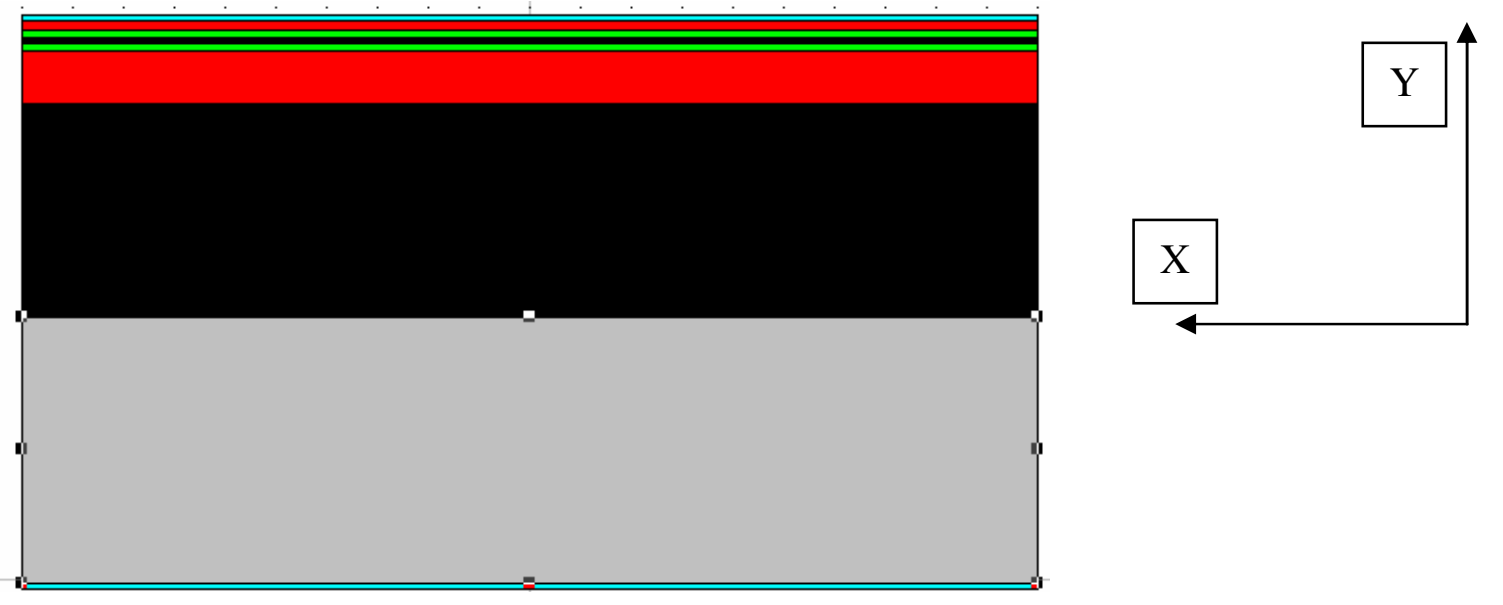

Figure 2-2: X and Y Direction of Model Laser Diode

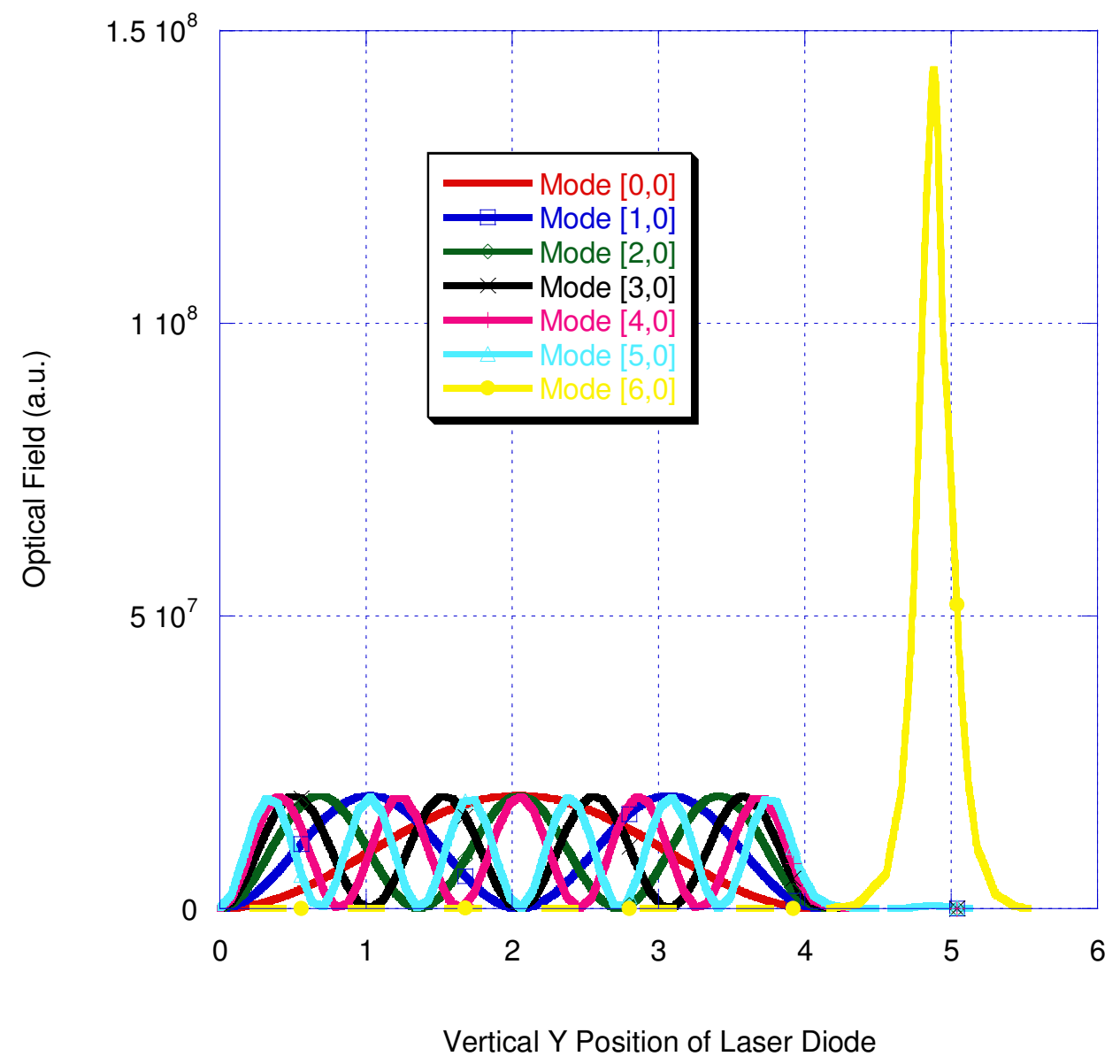

Figure 2-3: OCF's Dependence on Mode and Y Distance from Sapphire Layer 
Figure 2-3 shows a summary of all the modes aligned on the same optical field scale. It is apparent that the $6^{\text {th }}$ mode has an optical field intensity much greater than any of the other anti-guide modes. It is also important to notice that the anti-guide modes all have maximums at around the same intensities, which supports the existence of only one high optical intensity lasing mode. 


\subsection{Introduction to $1 D$ Optimization}

This chapter is focused on 1D design optimization. There are many variables that can be adjusted; however we chose to optimize the design by only focusing on changing layer thickness. Since the OCF is a good indication of how efficiently the light is being contained, the goal for optimization will be to maximize the OCF for the lasing mode. In the following simulations for the 1D model, the important layers will have their thicknesses adjusted to yield the best OCF. Once the OCF is maximized, the effects on the lasing threshold will be analyzed. 


\subsection{GaN Substrate Thickness Adjustment}

The first material adjusted is the GaN substrate layer. We adjust its thickness from $0-5 \mu \mathrm{m}$ and calculate the OCF for each of the first six modes.

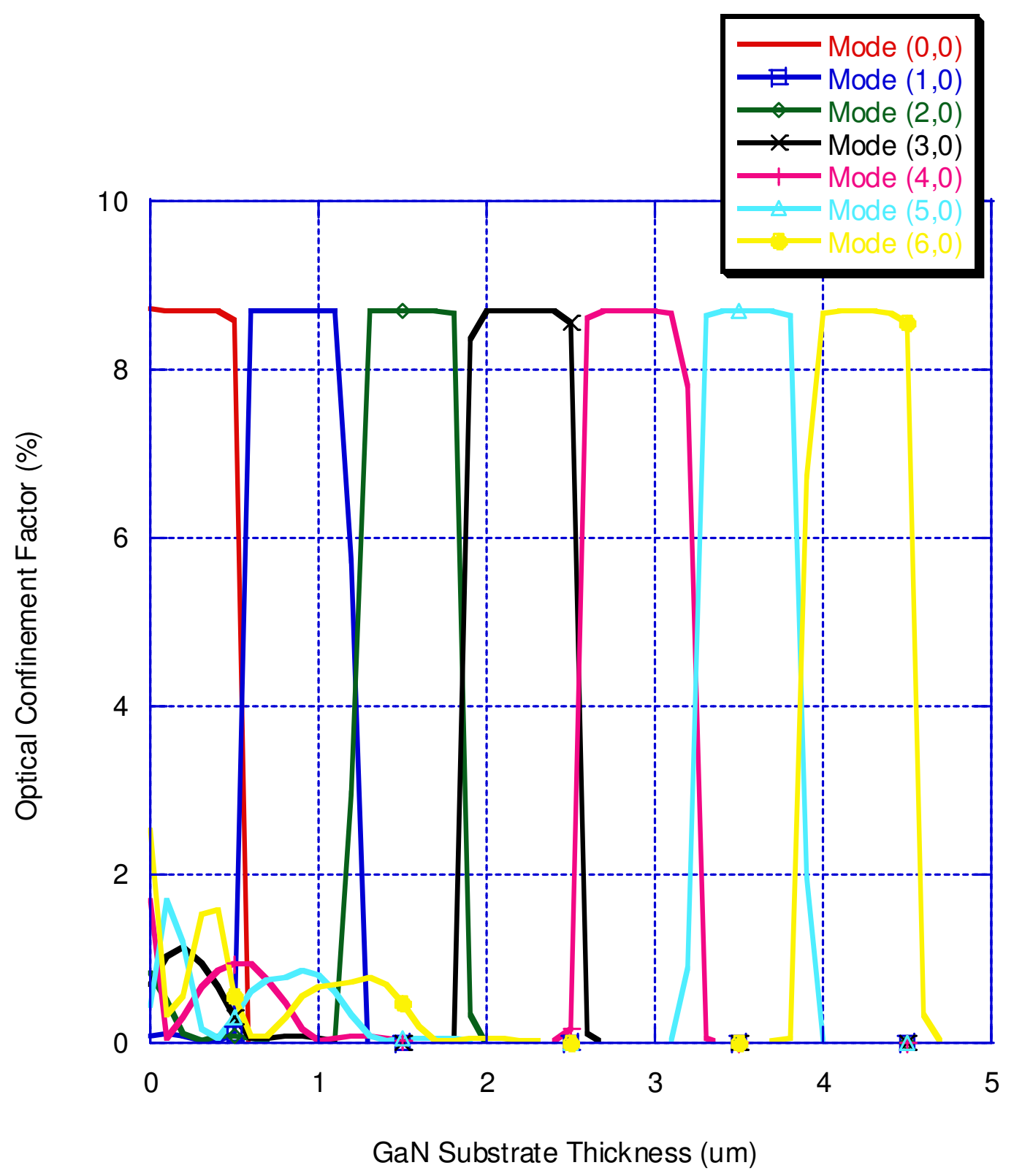

Figure 2-4: OCF vs. GaN Substrate Thickness 
In Figure 2-4 the substrate thickness is adjusted and the OCF is calculated. As the substrate thickness is changed, the mode with the greatest OCF changes. The mode with the greatest OCF is the lasing mode. When the lasing mode changes to a different mode, it is known as mode migration. Thus from Figure 2-4, an increase in the substrate results in the lasing mode migrating. These results have been supported by Einfeldt, Figge, Buttcher and Hommel. ${ }^{4}$ It is important to note that all the modes exist during all time, but the lasing mode dominates with the highest OCF.

Looking at Figure 2-4, the mode that is lasing will have its OCF abruptly drop as the substrate thickness is increased and a new mode will have an OCF that rises and becomes the new lasing mode. If a layer is designed to operate in the middle of these abrupt jumps the laser efficiency will be greatly degraded and result in laser instability. If a laser has a very small OCF, it will take an enormous amount of current to get the laser to emit light. An inefficient and unstable laser will ultimately lead to a shorter lifetime. Thus it is important to optimize a laser to its peak in the OCF.

Figure 2-4 shows that a nitride laser can be designed for a specific lasing mode by adjusting the GaN substrate. It would be ideal to design the GaN LD to have the fundamental mode as the lasing mode and relieve the laser of all the anti-guide modes in the GaN substrate. However this is not possible, because the GaN substrate must be of sufficient thickness to match the lattice constants of the sapphire and super-lattice cladding. Since the lasing mode can't occur at the fundamental mode, we can instead choose a substrate thickness that causes a peak in the OCF. Using our results it would be the best to choose a GaN thickness that has a peak in the OCF and is of sufficient thickness to allow proper lattice matching. Since at $4 \mu \mathrm{m}$ our laser isn't at the peak OCF, 
an easy adjustment would be to increase the thickness so that it falls more in the middle of the $6^{\text {th }}$ mode peak. With a GaN thickness of $4 \mu \mathrm{m}$ the OCF is $8.6677 \%$. By increasing the thickness to $4.1 \mu \mathrm{m}$ we can raise the OCF to $8.6933 \%$.

The migration of the lasing mode is very important in understanding the physics of what occurs in the GaN substrate layer. When Einfeldt, Figge, Buttcher and Hommel ${ }^{4}$ plotted the total OCF versus the GaN substrate thickness, each individual mode's OCF is not shown and thus it is ambiguous as to why the net OCF dips up and down. From our results we can see that this dip is directly related to the migration of the mode. The mode migration occurs at a constant increase in the GaN substrate. At every $0.8 \mu \mathrm{m}$ of thickness increase in the GaN substrate the lasing mode migrates. Thus when designing a nitride laser diode, if one can find this modal migration distance, lasers can be easily changed to have a different lasing mode by simply increasing the GaN thickness by a constant thickness.

\section{4 n-AlGaN (n-SL) and p-AlGaN (p-SL) Cladding Thickness Adjustment}

The cladding layer is an integral part of the waveguide structure in laser diodes. When using SLs as the cladding layer rather than a simple compound with a certain refractive index, many properties of the lattice can be modified. By modifying the alloy content and thickness of the thin layers in the SL the energy bandgap, resistance, crystal lattice and refractive index can be adjusted. Thus using SLs for the cladding layer allow the designer to more meticulously modify the cladding layer. In this simulation, only the thickness of the n-SL cladding layer is adjusted and the OCF is calculated for each mode. 
It is expected that an increased cladding layer thickness will result in a better OCF, because a thicker cladding layer reduces the effects of the outer contact layer. ${ }^{12}$

Figure 2-5 shows the effects of increasing the n-doped cladding layer to eliminate the non-lasing energy at the $6^{\text {th }}$ mode (the lasing mode). When the cladding layer is increased from $0.15 \mu \mathrm{m}$ to $0.8 \mu \mathrm{m}$, the non-lasing energy is eliminated and at $0.8 \mu \mathrm{m}$ most all the energy is confined in the single hump located at the position of the quantum well. Thus it is important to have a cladding layer of sufficient thickness to confine the energy into the single lasing mode in the quantum well. 


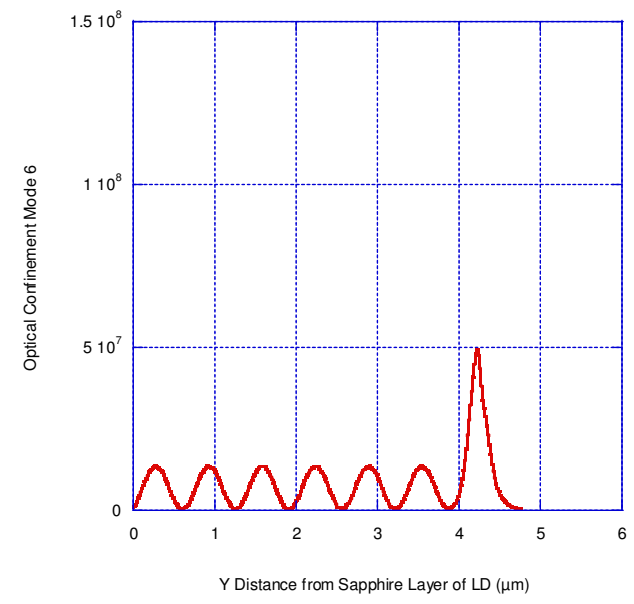

(a)

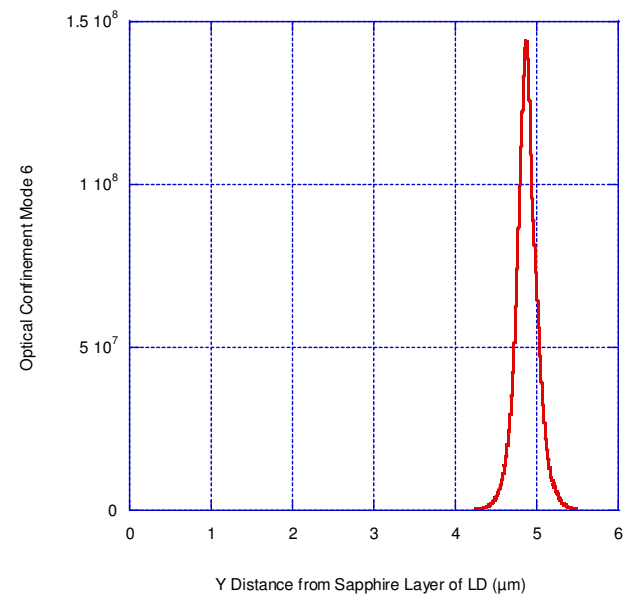

(b)

Figure 2-5: Optical Confinement Factor vs. Y Distance from Sapphire layer at Mode 6 for n-SL Thicknesses of a) $0.15 \mu \mathrm{m}$, and b) $0.8 \mu \mathrm{m}$
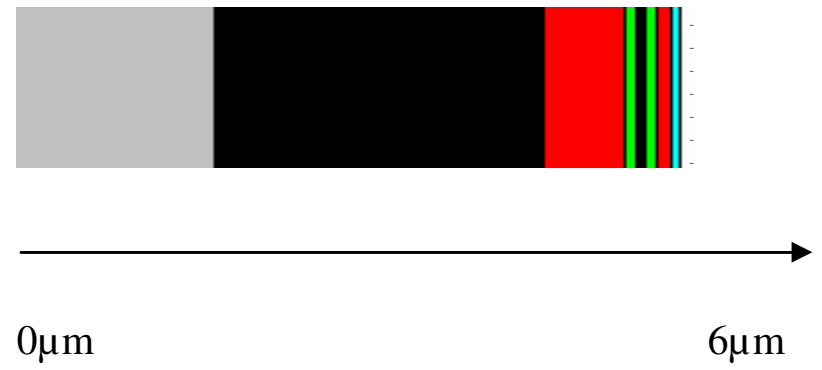

Figure 2-6: Y-Axis shown in comparison to different layers

Increasing the cladding layer thickness increases the OCF, so there should be an optimum cladding thickness that leads to a large OCF and isn't too thick so that manufacturing the layer is impossible. To find the optimum thickness the n-AlGaN cladding layer was adjusted from $0-1.5 \mu \mathrm{m}$ and plotted against the OCFs. The results of 
this calculation are shown in Figure 2-7. From Figure 2-7 it can be seen that the highest OCF is $8.74265 \%$ and occurs at an $\mathrm{n}-\mathrm{AlGaN}$ thickness of $1.5 \mu \mathrm{m}$. However it is very hard to create such a thick cladding layer in the real world. The thicker the cladding becomes, the more defects occur and thus lead to undesirable properties in the layer. Since the program I am using does not take into account the crystal growth processes and techniques, the program assumes a perfect $1.5 \mu \mathrm{m}$ layer is possible and optimal. However in the actual fabrication process, there is no technique to deposit such a thick layer and have it function properly. The original $\mathrm{n}-\mathrm{AlGaN}$ layer was $0.8 \mu \mathrm{m}$ which yielded an OCF of $8.6997 \%$. This OCF is only approximately $0.4 \%$ less than the $1.5 \mu \mathrm{m}$ thick cladding layer OCF of $8.74265 \%$ and has a thickness that is much more in range for real world crystal growth.

Judging by Figure 2-7 at a thickness of $0.8 \mu \mathrm{m}$, a few nanometers increase in the thickness of the n-AlGaN will not greatly increase the OCF. However, a few nanometers thinner will begin to have drastic negative effects on the OCF. Thus it can be concluded that $0.8 \mu \mathrm{m}$ is an optimal thickness for the n-SL layer, but no thinner. 


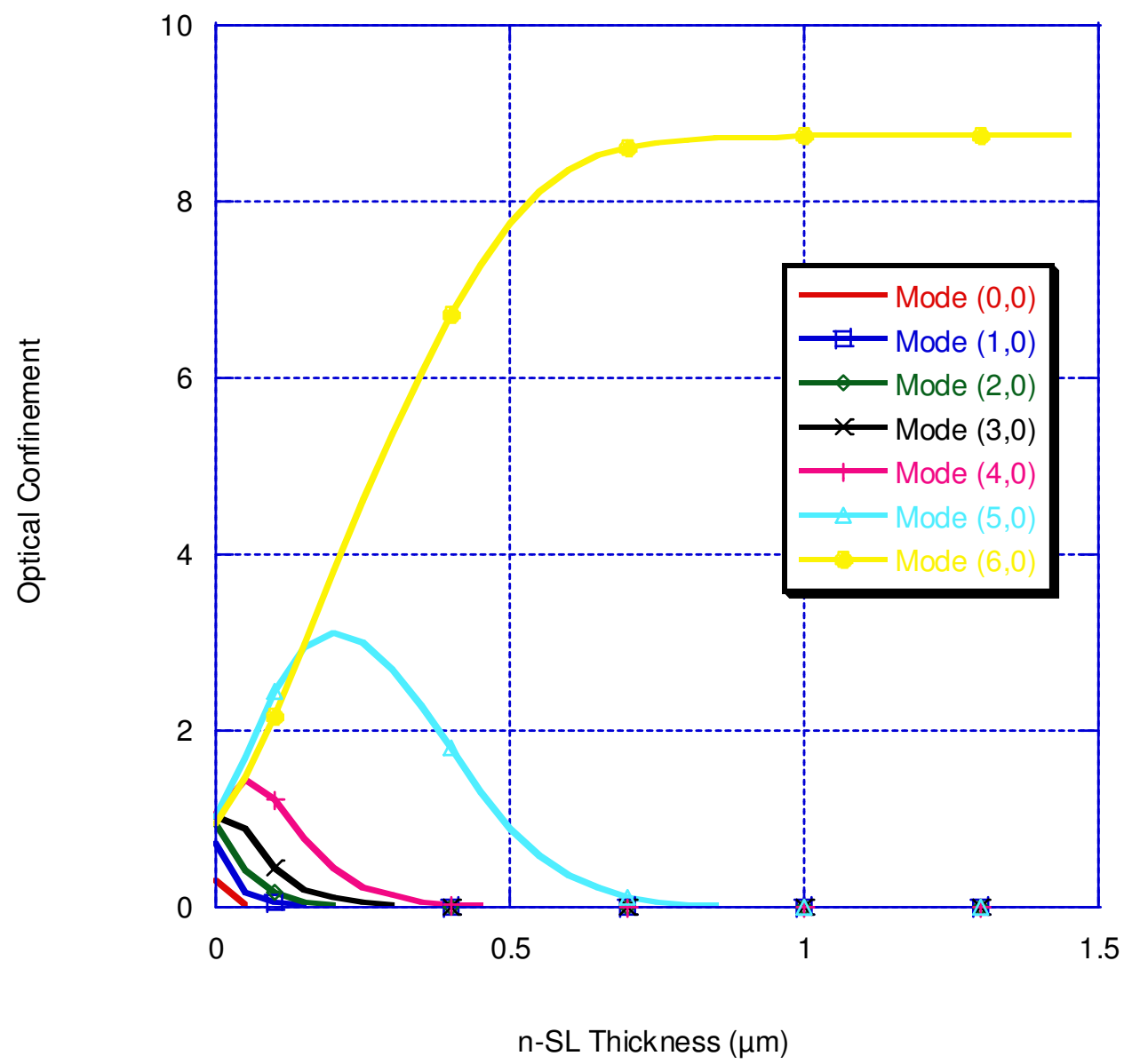

Figure 2-7: Optical Confinement vs. n-SL Thickness

After discovering that $0.8 \mu \mathrm{m}$ was the optimal thickness for the n-SL layer, the next step was to find the optimal layer thickness for the p-SL layer. The thickness of the p-SL layer was adjusted from 0-1.5 $\mu \mathrm{m}$ and plotted against the OCF in Figure 2-8. Figure 2-8 resembles a very different outcome than that of Figure 2-7. The OCF reaches a very early maximum at $0.1 \mu \mathrm{m}$ and then begins a quick decline and reaching an asymptotic value of $8.4 \%$. Thus a value of $0.1 \mu \mathrm{m}$ for the $\mathrm{p}$-SL would result in the highest OCF of 8.7\%. The p-SL prevents light from leaking into the upper portion of the laser diode or more specifically into the $\mathrm{p}-\mathrm{GaN}$ contact. It is interesting that a very small thickness 
results in the best OCF considering a thicker cladding usually is best at blocking light from entering other parts of the laser diode. However, when designing a GaN LD, it is important to have a thin p-SL layer so current can be effectively conducted through the diode.

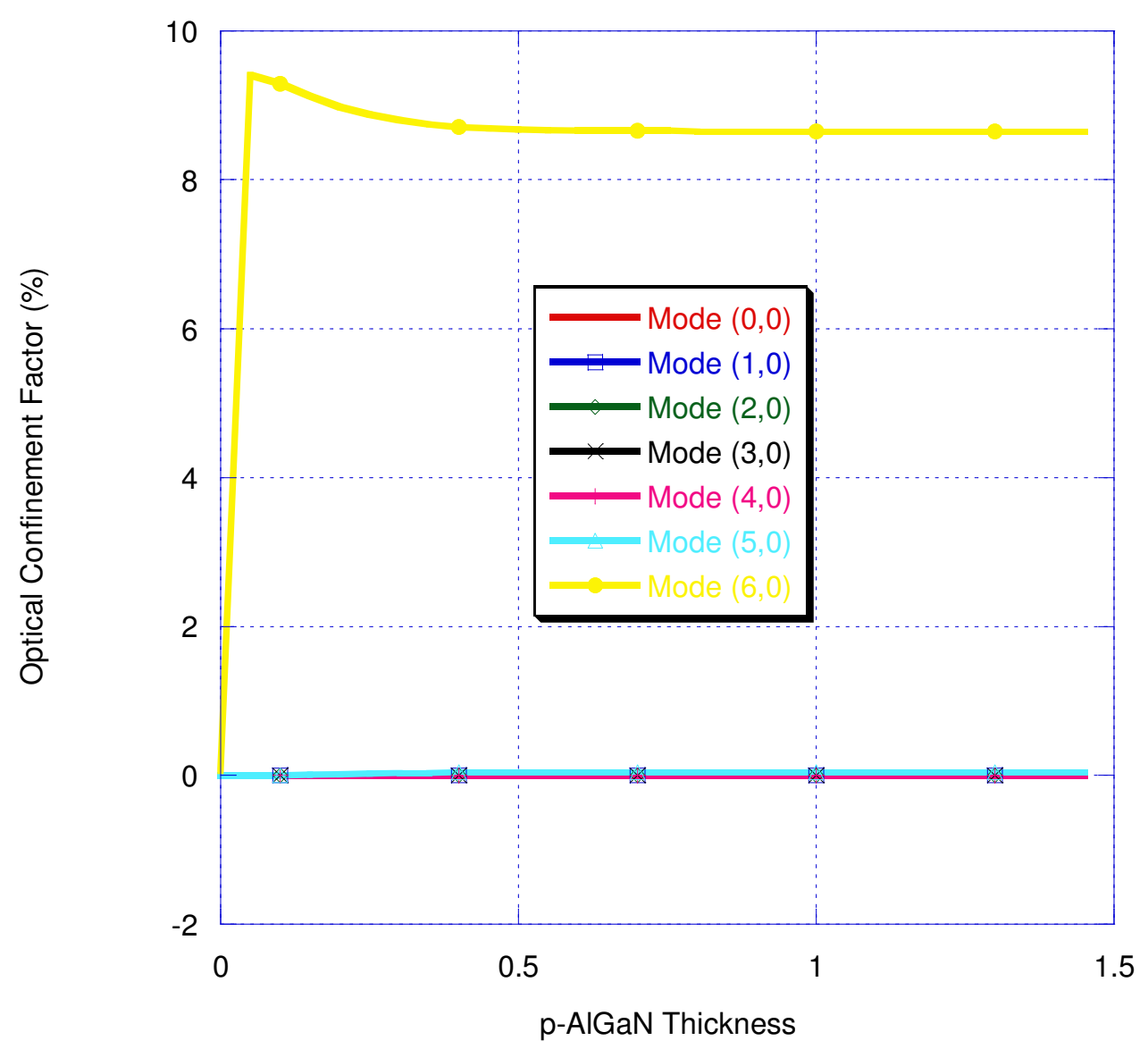

Figure 2-8: Optical Confinement vs. p-SL Thickness 


\section{5 n-GaN and p-GaN Cladding Thickness Adjustment}

Increasing the cladding thickness should result in better optical confinement because the light will not as easily escape with a thick confining layer. Thus it is important to analyze for what thickness of n-GaN will result in optimal optical confinement. It is also important to note that if the cladding layer is too thin, then light will easily escape through this thin barrier and lead to very bad optical confinement.

In this simulation all other layers are kept constant and adjusted the $\mathrm{n}-\mathrm{GaN}$ cladding layer thickness is adjusted. Figure 2-10 shows the results of this experimental simulation. For very small values of $\mathrm{n}-\mathrm{GaN}$ thickness up to $0.05 \mu \mathrm{m}$, there is no confinement of the $6^{\text {th }}$ mode. In fact there is no confinement for any mode, revealing that a thicker cladding layer is necessary in creating the lasing action. At an n-GaN thickness of $0.05 \mu \mathrm{m}$, the OCF jumps to $8.456 \%$ and reaches a peak OCF at $0.075 \mu \mathrm{m}$ of $8.7175 \%$. As the thickness of the n-GaN layer goes beyond $0.1 \mu \mathrm{m}$ there is a sudden drop off of the OCF for the $6^{\text {th }}$ mode and the laser begins lasing in the $5^{\text {th }}$ mode. The n-GaN thickness of $0.075 \mu \mathrm{m}$ is a $0.5 \%$ increase in the OCF over the original $\mathrm{n}-\mathrm{GaN}$ thickness of $0.1 \mu \mathrm{m}$ which yielded an OCF of 8.67\%. The shape and abrupt drops in the OCF when the lasing mode migrates from the $6^{\text {th }}$ to $5^{\text {th }}$ mode is very similar to those of Bergmann and Casey. The highest OCF can be attributed to the thickness of $\mathrm{n}-\mathrm{GaN}$ for which resonance occurs in the active region. ${ }^{14}$

It is interesting to note that as the thickness of the $\mathrm{n}-\mathrm{GaN}$ layer gets larger the lasing mode migrates to lower modes. This is the opposite of what happened in the GaN substrate simulation. The larger $\mathrm{n}-\mathrm{GaN}$ core thickness allows less light into the substrate mode and therefore the large thickness of the substrate mode does not have as strong 
effect on creating ghost modes. Since less ghost modes are occurring, the lasing happens at lower modes. From looking at Figure 2-10 there is a noticeable slope at which the OCF degrades. Understanding the slope decrease will be an opportunity for future work.

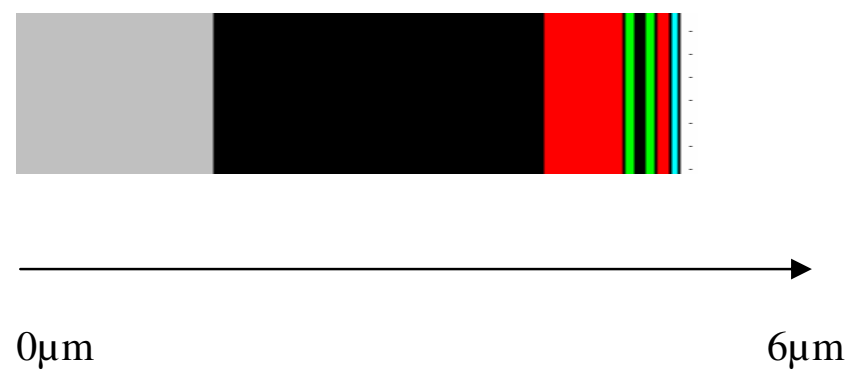

Figure 2-9: Y-Axis shown in comparison to different layers 


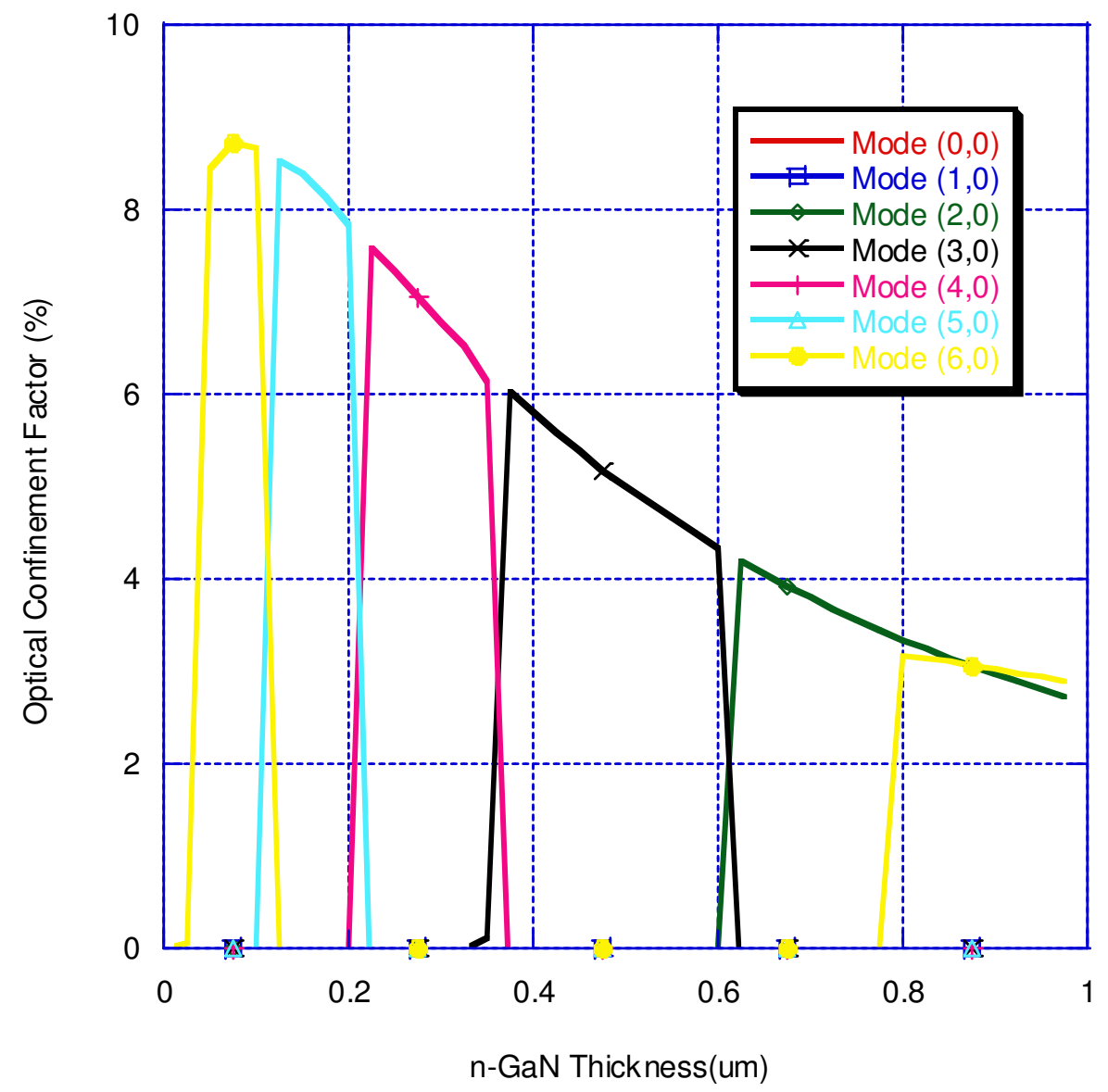

Figure 2-10: Optical Confinement vs. n-GaN Core Layer Thickness

Analysis of the $\mathrm{p}-\mathrm{GaN}$ cladding layer follows very similarly to that of the n-GaN cladding layer. The higher refractive index backed by a lower cladding refractive index confines the light in the active region. Layered on top of a very thin e-block, the p-GaN core layer is the second to last step in the waveguide structure - the final step being the pdoped cladding layer. 
Figure 2-11 shows a similar outcome to that of the $n-G a N$ 1D simulation in Figure 2-10 and to the results in [14]. For too thin of a p-GaN layer, there is no optical confinement at all and for too thick of a p-GaN layer, the lasing mode migrates to lower modes. It shows that the optimal thickness for the largest confinement factor when lasing in the $6^{\text {th }}$ mode is $0.05 \mu \mathrm{m}$. This is $50 \mathrm{~nm}$ less than what is in our original design for the LD structure. With a $\mathrm{p}-\mathrm{GaN}$ thickness of $0.05 \mu \mathrm{m}$ the OCF is $8.87 \%$, compared to an original OCF for a $\mathrm{p}-\mathrm{GaN}$ thickness of $0.1 \mu \mathrm{m}$ of $8.67 \%$. Thus by doing this simulation we have found a way to increase the OCF by $0.2 \%$. Though it is a very small amount, hopefully the optimization of each layer thickness will lead to a larger overall increase in the OCF and thus yield a laser diode with a lower lasing threshold. 


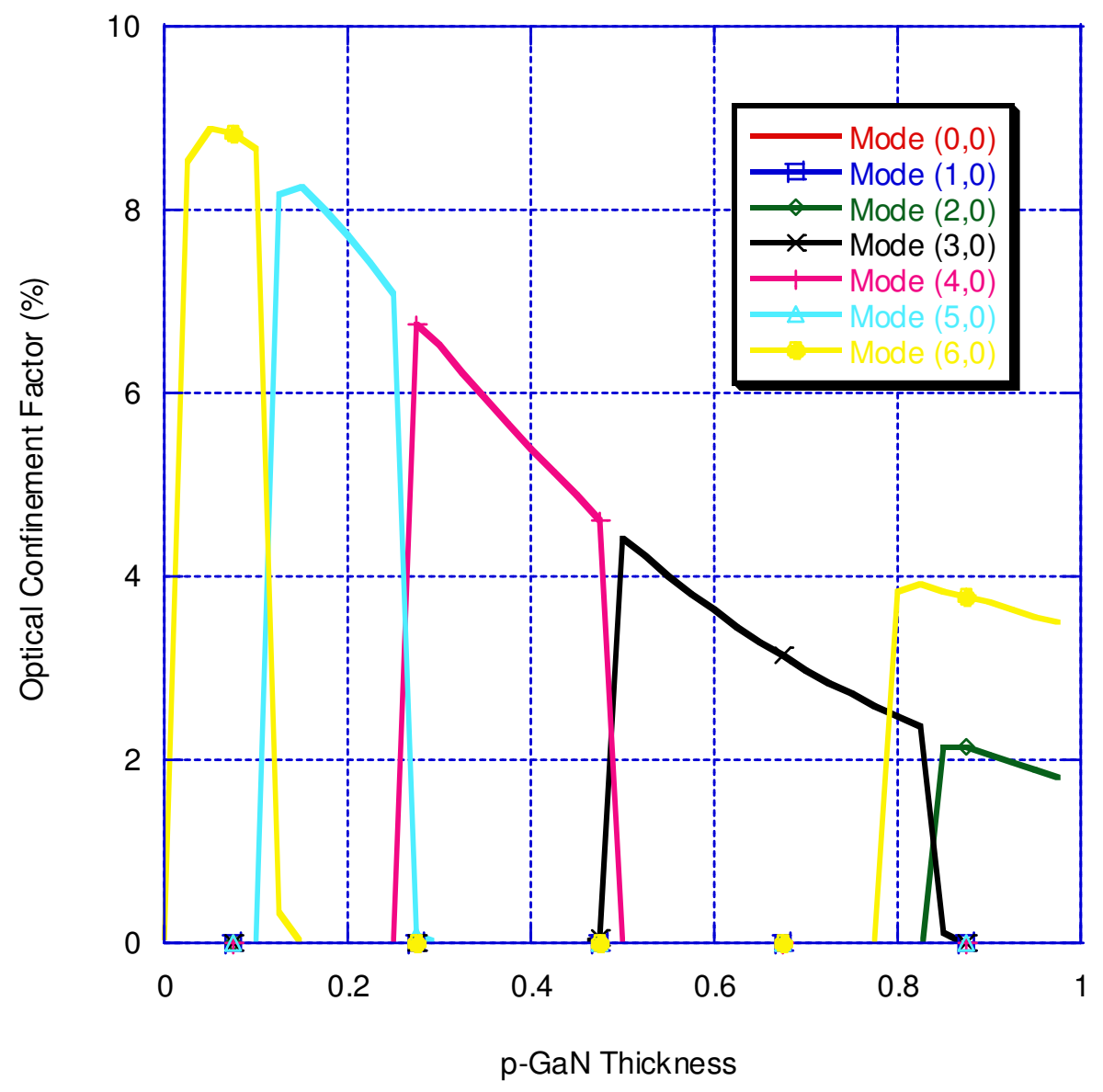

Figure 2-11: Optical Confinement vs. p-GaN Core Layer Thickness 


\section{6 p-GaN Contact Thickness Adjustment}

The contact layers are located at the ends of the LD. They provide the connection to the metal-electrode used to apply a voltage across the laser diode. The $\mathrm{n}-\mathrm{GaN}$ contact layer is also known as the substrate and is the thickest layer in the laser diode. The p-GaN layer however is fabricated to be thin to maintain a small series resistance and provide adequate current injection for lasing to occur. In this optimization simulation the effects of different p-GaN thickness on the OCF is observed. Since the light is confined in the active region or confined in the GaN substrate buffer (in the anti-guide case), any increase in p-GaN contact thickness should not adversely affect the OCF. Figure 2-12 confirms that $\mathrm{p}-\mathrm{GaN}$ contact thickness does not dramatically affect the OCF. The lasing mode shown as the yellow line maintains a high OCF at around $8.6 \%$ and is very constant over the $200 \mathrm{~nm}$ change in the $\mathrm{p}-\mathrm{GaN}$ contact thickness. 


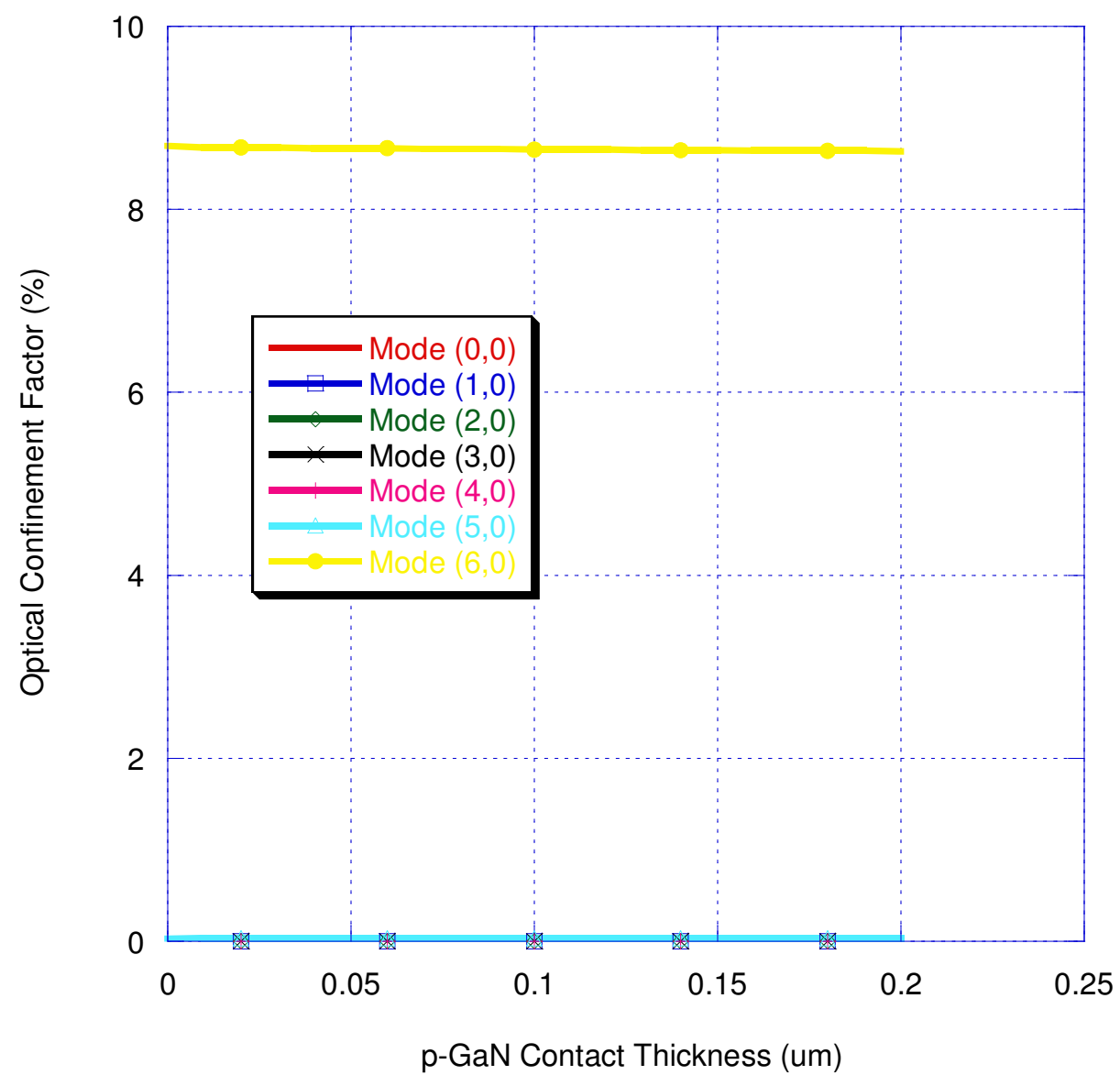

Figure 2-12: Optical Confinement vs. p-GaN Contact Thickness 


\section{CHAPTER 3: OPTICAL MODE 2D SIMULATIONS}

\subsection{Introduction to 2D Optimization}

After simulating the GaN LD design in the 1D mode it was discovered that there were optimum thicknesses of each layer which maximized the optical confinement factor. In this Chapter, similar simulations will be performed on the 2D design LD. The 2D simulation should closely follow the results of the 1D simulation results and also provide information on how the optical modes interact in the $2 \mathrm{D}$.

The optical modes of the 2D simulation are shown in Figure 3-1. For modes $(0,0)$ through $(7,0)$ the modes look very similar to the $1 \mathrm{D}$ results, with additional modal energy being layered above and below adjacent energies. The $8^{\text {th }}$ mode is the lasing mode and shows the optical energy confined inside the active region. Modes $(9,0)$ and $(10,0)$ continue the same trend of optical energy layering as modes 1 through 7 . However, something very interesting happens in mode $(11,0)$. Instead of the modal energy continuing its vertical layering trend the modal energy splits across the $\mathrm{x}$-axis horizontally. The next modes- $(12,0),(13,0),(15,0),(16,0),(18,0)$ and $(20,0)$ follow this new horizontal trend. So we notice two modal patterns: A single vertical layering of optical energy, and also split of energy across the horizontal axis with double vertical layering of optical energy. The double layering is a characteristic that we would not be able to notice without doing a 2D model and gives insight into the optical energy interaction along the $\mathrm{x}$-axis. We find that as the mode increases to the optical energies are no longer contained along the vertical boundaries and the energies split and are shared across the $\mathrm{x}$-axis. 


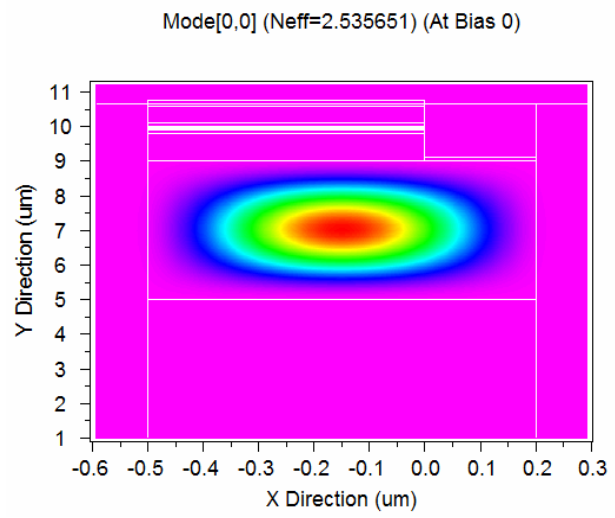

(a)

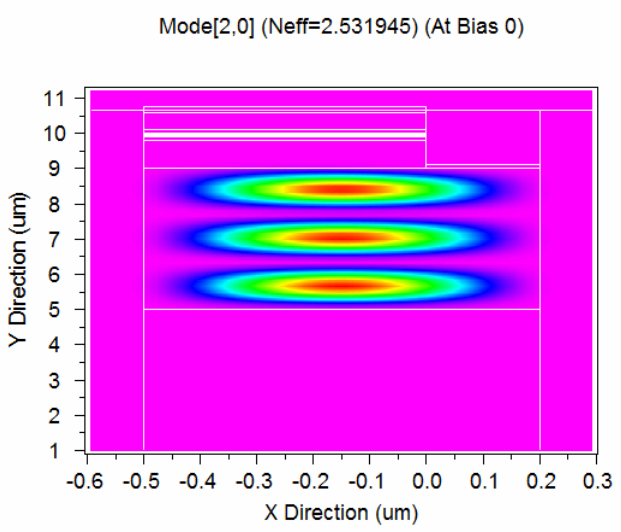

(c)

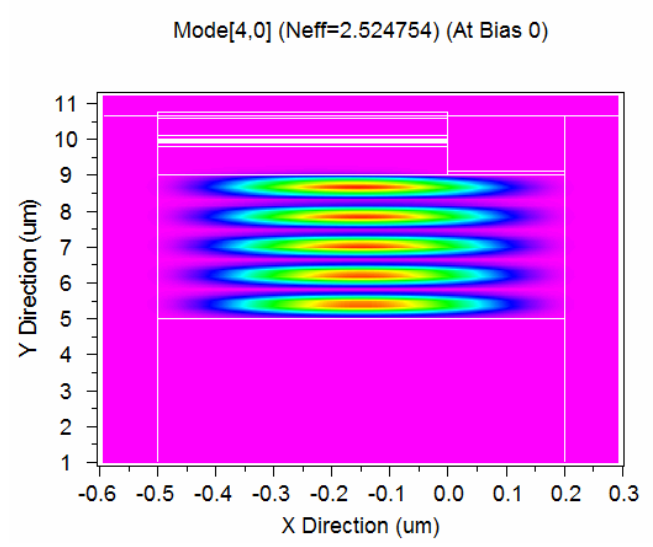

(e)

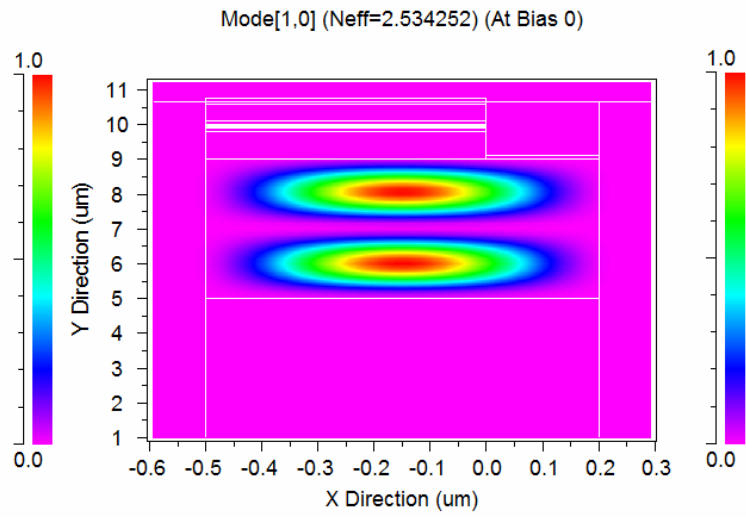

(b)
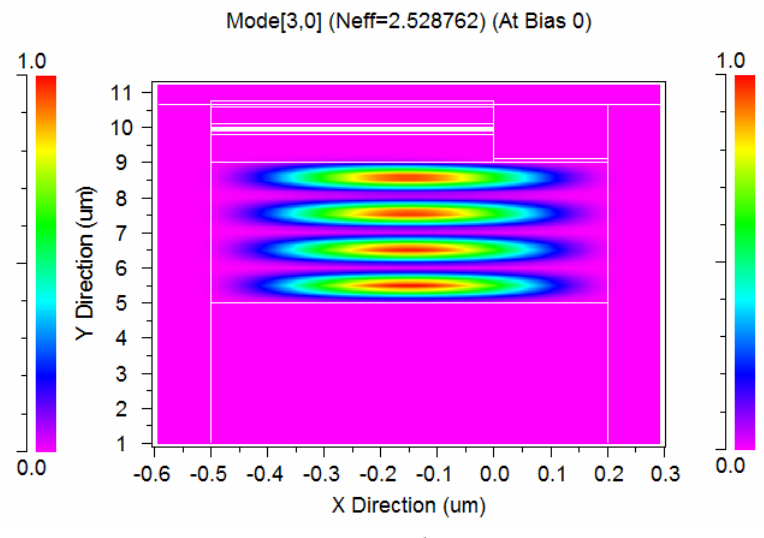

(d)
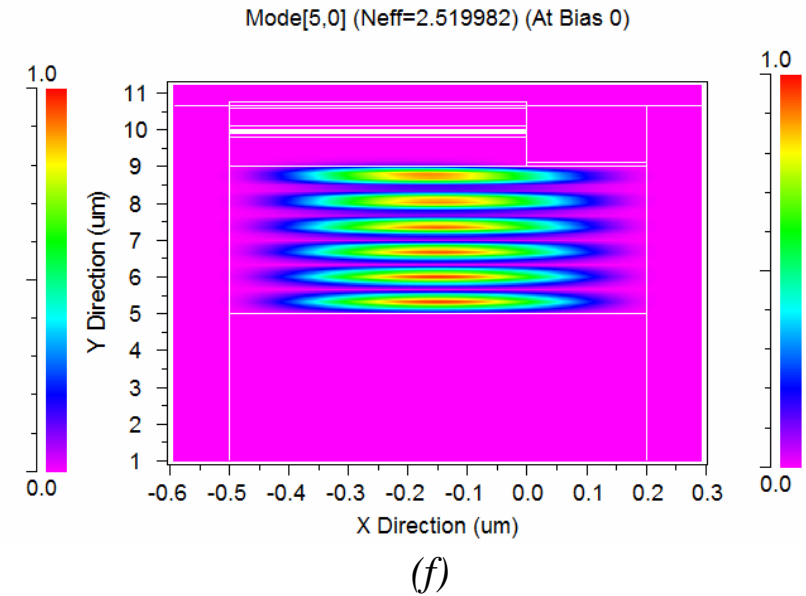


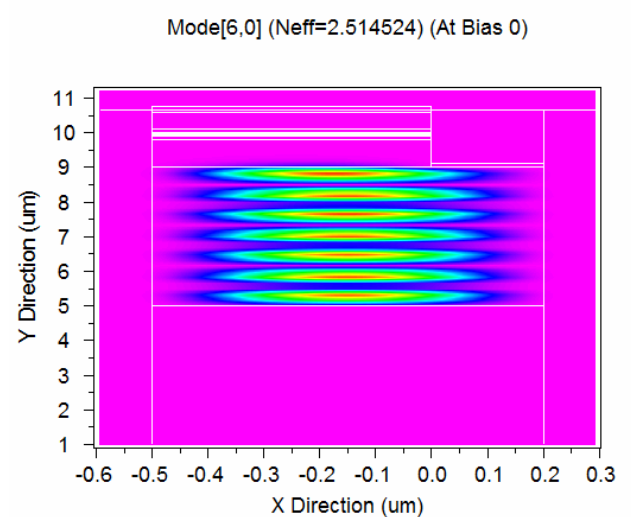

(g)

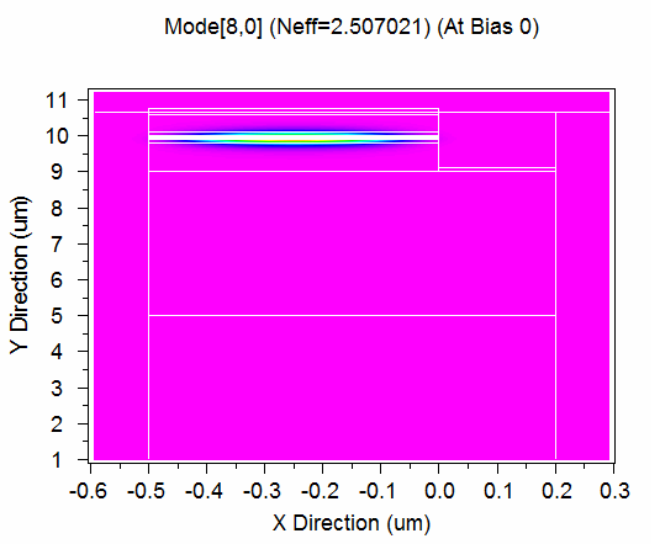

(i)

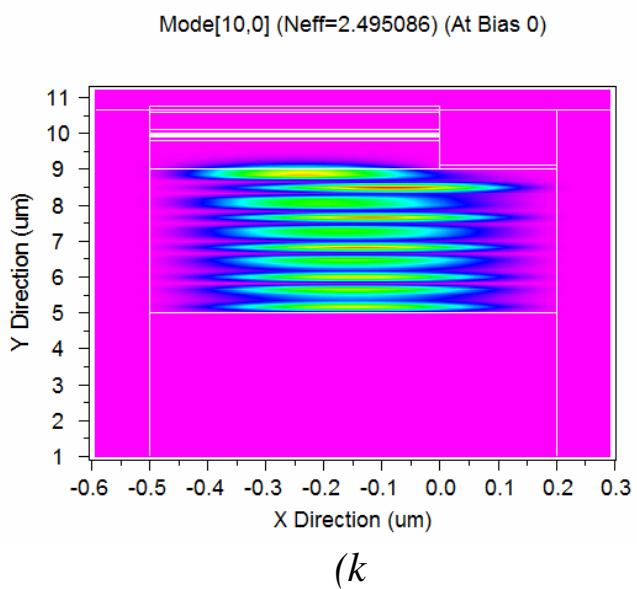

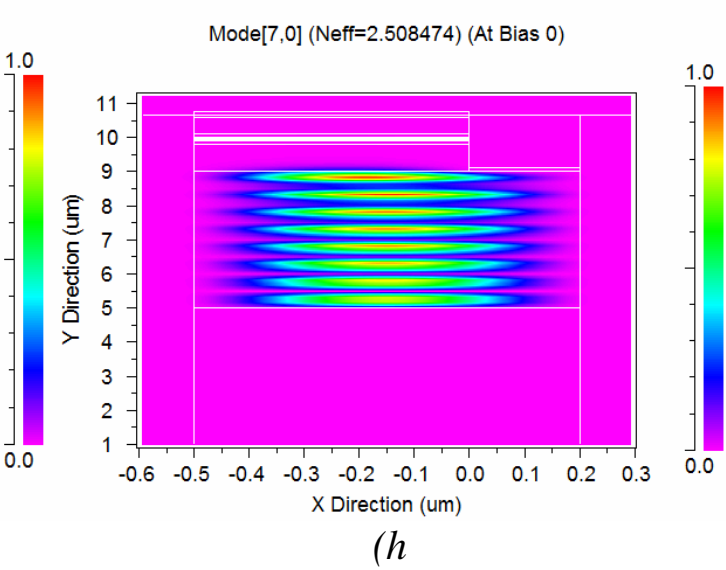
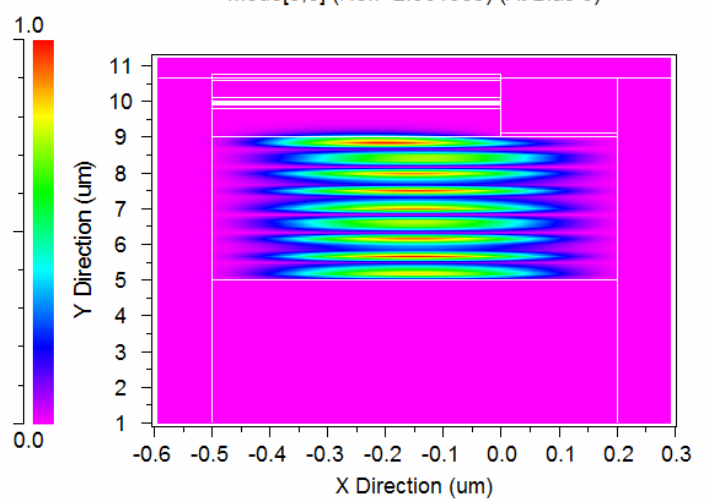

(j)

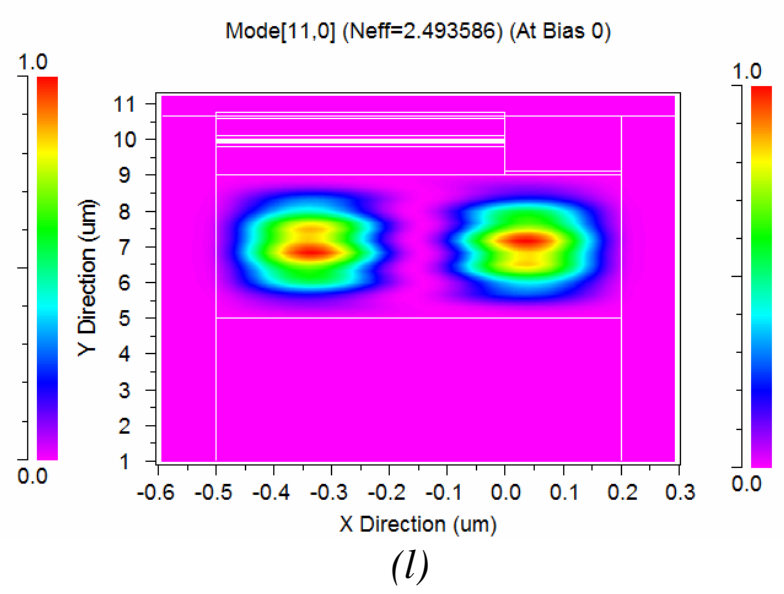


Mode $[12,0]($ Neff $=2.492153)($ At Bias 0$)$

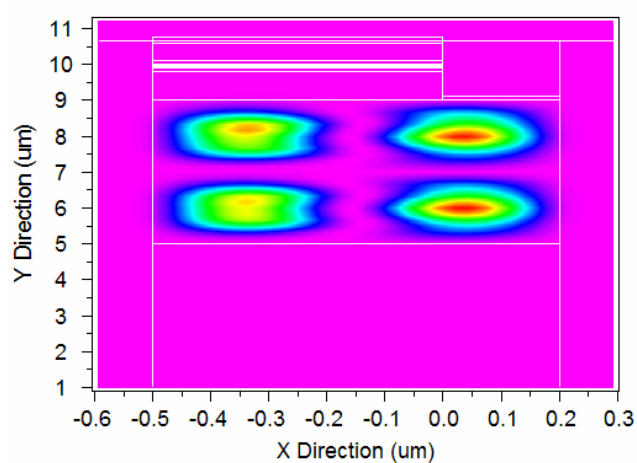

(m)

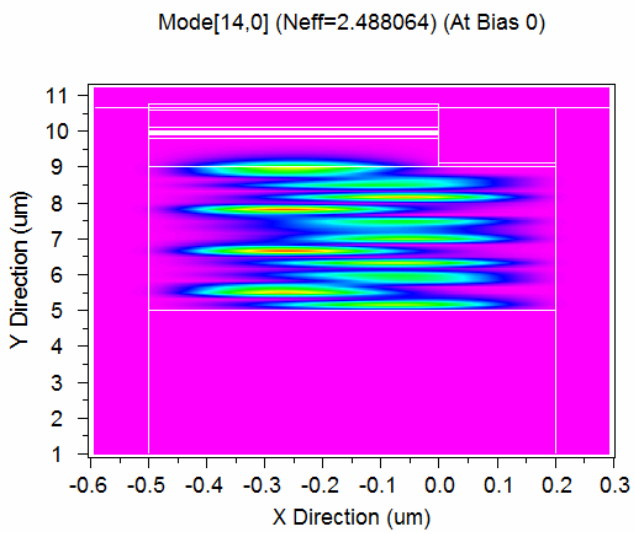

(o)

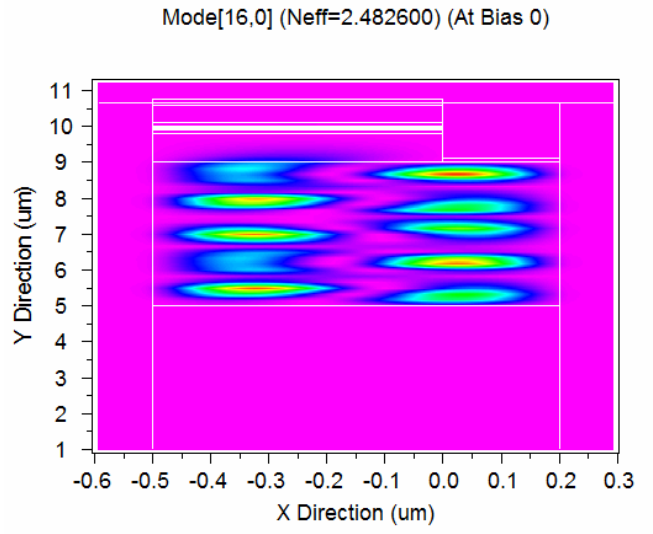

(q)
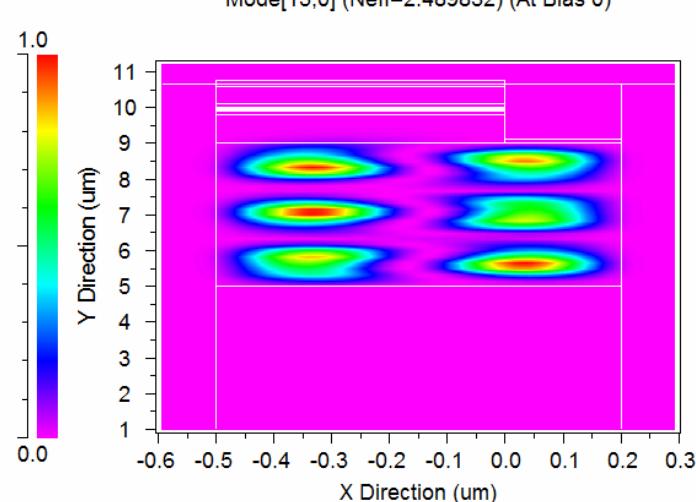

(n)
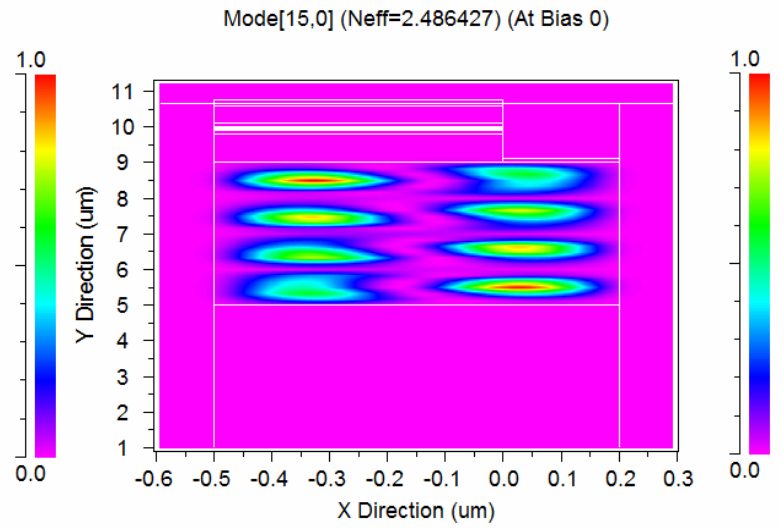

(p)
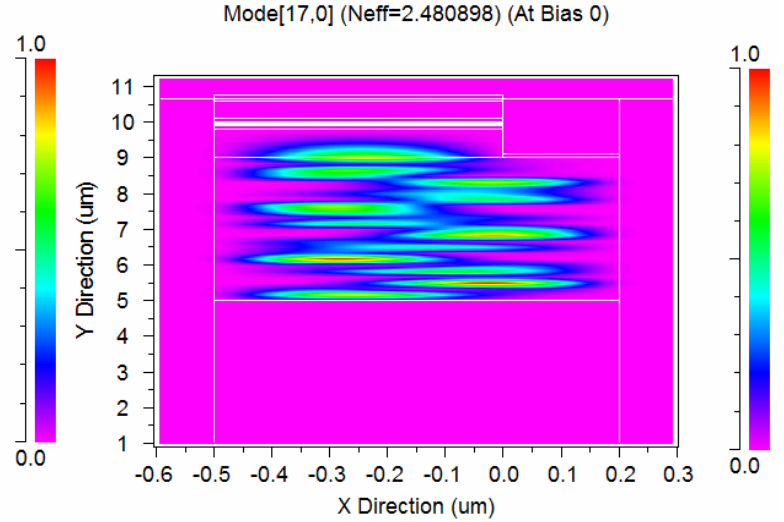

$(r)$ 


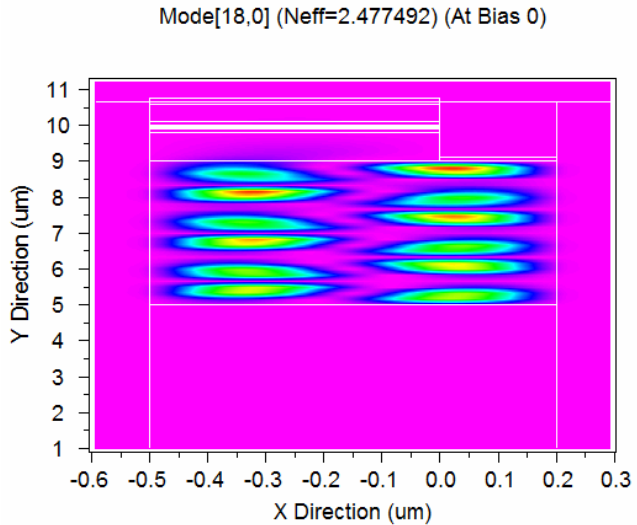

(s)

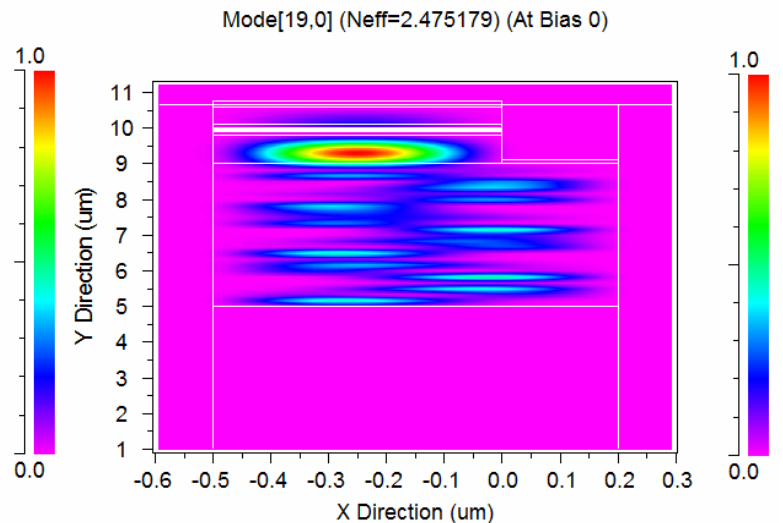

$(t)$

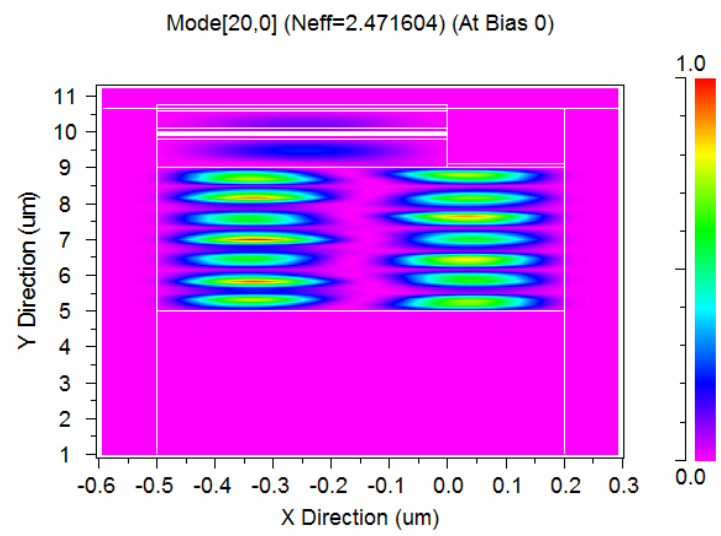

(u)

Figure 3-1: 2D Plots of Optical Confinement vs. X and Y Distance for (a) $0^{\text {th }}$ mode (b) $1^{\text {st }}$ mode (c) $2^{\text {nd }}$ mode (d) $3^{\text {rd }}$ mode (e) $4^{\text {th }}$ mode (f) $5^{\text {th }}$ mode $(\mathrm{g}) 6^{\text {th }}$ mode- lasing (h) $7^{\text {th }}$ mode (i) $8^{\text {th }}$ mode (j) $9^{\text {th }}$ mode $(\mathrm{k}) 10^{\text {th }}$ mode (l) $11^{\text {th }}$ mode $(\mathrm{m}) 12^{\text {th }}$ mode $(\mathrm{n}) 13^{\text {th }}$ mode (o) $14^{\text {th }}$ mode (p) $15^{\text {th }}$ mode (q) $16^{\text {th }}$ mode- lasing (r) $17^{\text {th }}$ mode (s) $18^{\text {th }}$ mode (t) $19^{\text {th }}$ mode, and (u) $20^{\text {th }}$. 


\subsection{GaN Buffer Thickness Adjust}

When the 2D design was simulated with the original design from Table 1-2 there was an interesting different between the $1 \mathrm{D}$ and $2 \mathrm{D}$ results. While the $1 \mathrm{D}$ had done its lasing in the $6^{\text {th }}$ mode, the $2 \mathrm{D}$ did its lasing in the $8^{\text {th }}$ mode. This change can be attributed to the change in the design of the LD when modifying from a $1 \mathrm{D}$ design to a $2 \mathrm{D}$ design. In the $2 \mathrm{D}$ design the $\mathrm{LD}$ is no longer completely symmetrical so it is expected the $1 \mathrm{D}$ and 2D will not share the exact same measurements. However it is expected that the results will follow the same general trend.

The OCF is measured in the 2D design for GaN substrate layer thicknesses of from $0-5 \mu \mathrm{m}$. Figure 3-2 shows the values of the OCFs measured and for what mode they occurred. The lasing migrates as the GaN thickness increases. For every increase in the GaN substrate by $0.5 \mu \mathrm{m}$ the lasing mode jumps up a mode. For example, observing Figure 3-2 at a GaN thickness of $2.6 \mu \mathrm{m}$, the lasing mode is $5^{\text {th }}$ mode, but increasing the GaN thickness to $3.1 \mu \mathrm{m}$ the lasing mode migrates to the $6^{\text {th }}$ mode. The amount of width $(\mu \mathrm{m})$ that the lasing action spends in each mode is equal and thus each mode is evenly spaced by the constant of $0.5 \mu \mathrm{m}$. These results are in agreement with our previous 1D GaN substrate simulation results and with ${ }^{4}$. 


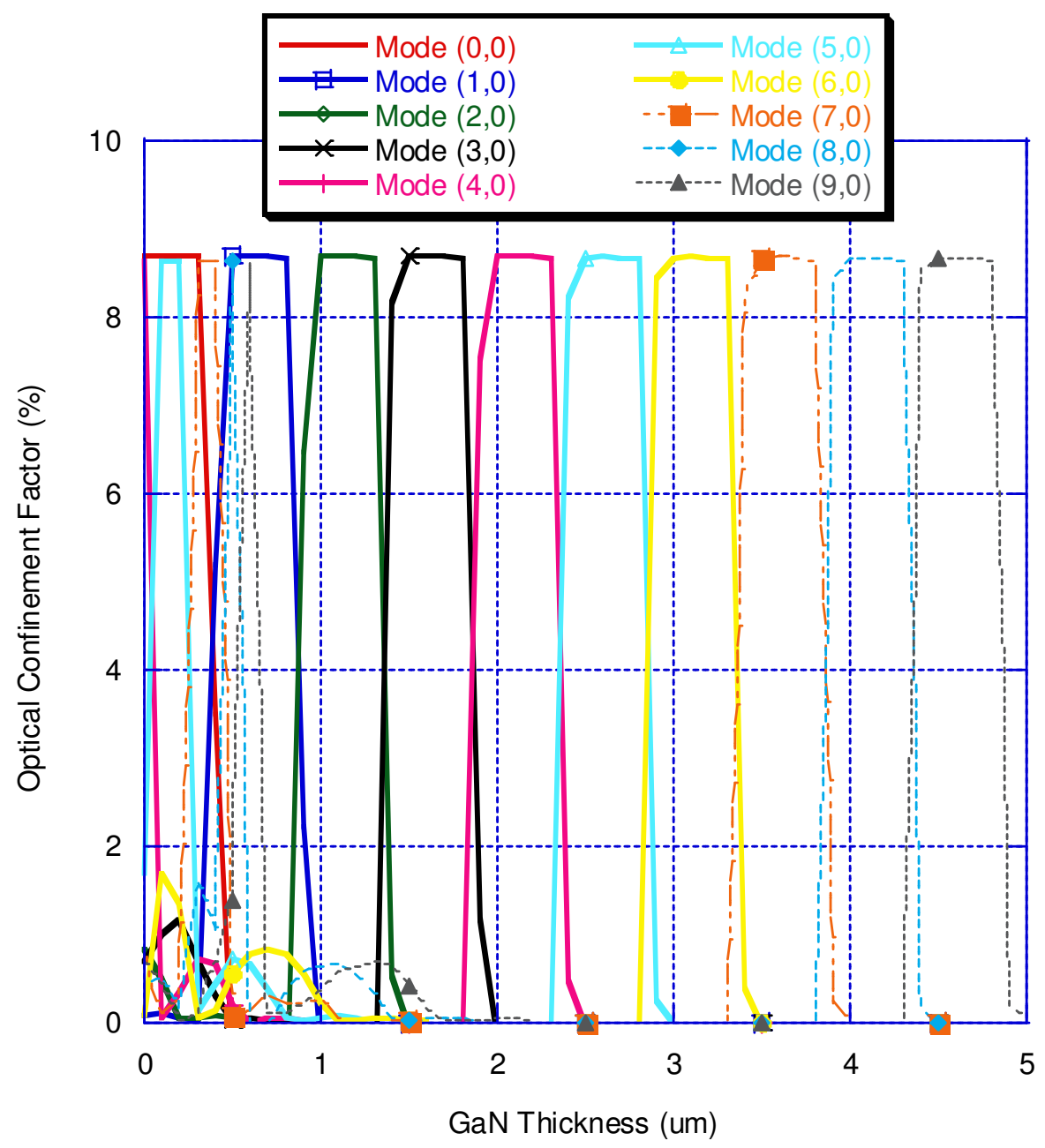

Figure 3-2: OCF vs. GaN Substrate Thickness (2D)

Using the original design from Table 1-2, the simulated OCF for the lasing mode ( $8^{\text {th }}$ mode) is $8.6677 \%$. However, in Figure 3-2, the OCF can still be increased if the GaN thickness is increased. Following along the line that represents the $8^{\text {th }}$ mode, a thickness of $4.2 \mu \mathrm{m}$ yields an OCF of $8.6962 \%$ which is a $0.02 \%$ increase over the original design. 
Only a small increase in the OCF was possible for the GaN substrate layer showing that the original layer thickness was very close to being an optimal design. 


\section{3 n-AlGaN and p-AlGaN SL Cladding Thickness Adjust}

It is expected that increasing the cladding layer thickness will lengthen the distance that the light has to tunnel through in order to get into the substrate waveguide. This will prevent optical leakage of the mode into the absorbing, high index p-contact layer. This however will increase the impedance as it would also lengthen the distance the current would have to tunnel through and therefore increase our threshold current. ${ }^{15}$ By adjusting the thickness in the n-SL it is of interest to find a smaller thickness that still retains a high OCF and a thickness that prevents mode and current leakage. It is also important to be aware that creating such thin coats of the n-SL still remains a real world problem and although a thin coat of n-SL may lead to an optimized simulation, creating such a lasing device may be troublesome. By increasing the cladding size, we are looking to find how much a thickened cladding actually affects the OCF. We expect a thickening of the n-SL will prevent mode leakage from reaching the substrate and thus help prevent ghost modes therefore leading to a higher OCF. 


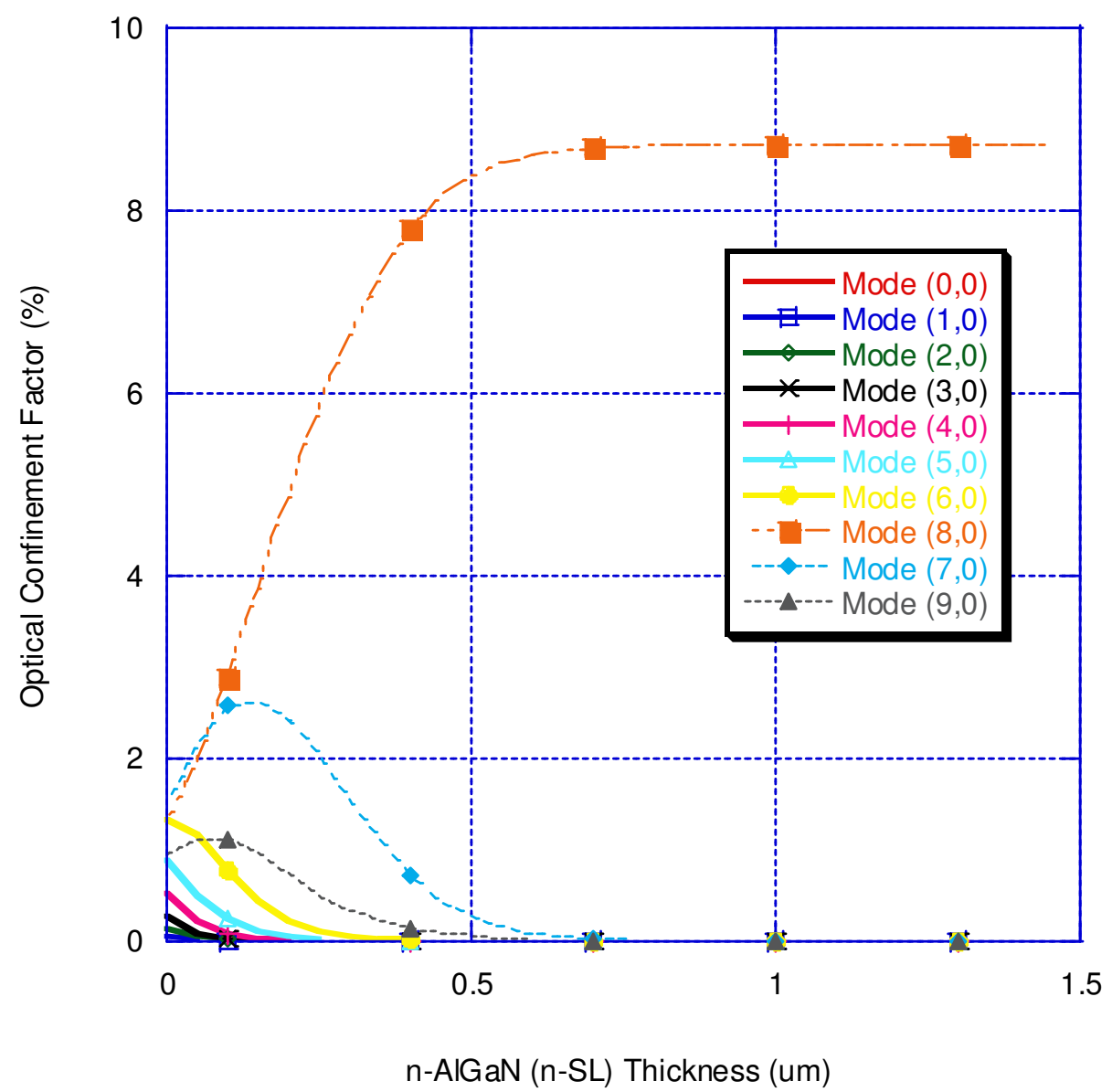

Figure 3-3: Optical Confinement vs. n-SL Thickness

The results of the 2D simulation with varying n-SL thickness in Figure 3-3 are similar to those Hatakoshi calculated. ${ }^{12}$ As the cladding thickness is increased the $8^{\text {th }}$ mode (lasing mode) gains begins to confine more light. As the lasing mode increases in its OCF, the other modes begin to lose the light which they had originally been able to confine. Thus as the cladding thickness increases, all the optical energy that is available 
becomes confined only in the $8^{\text {th }}$ mode. This is shown in Figure 3-3. This result shows that the anti-guide-like or ghost mode behavior can be suppressed by increasing the cladding layer thickness in agreement with Hatakoshi. ${ }^{12}$ A thick cladding layer reduces the effect of the outer contact layers, but still is conducive to high-order modes.

At the $\mathrm{n}$-SL thickness of the original design $(0.8 \mu \mathrm{m})$, the OCF was $8.7128 \%$ and reaches a peak plateau on the graph at $8.7241 \%$ at a thickness of $1.4 \mu \mathrm{m}$. The $0.01 \%$ increase in OCF requires almost doubling the thickness. Thus the best solution for this design is simply leaving it alone. The original design yields a reasonable OCF value.

The p-SL cladding provides the same waveguide like confinement of the light as the n-SL cladding, but it differs in that it will not be absorbing electrons like the p-SL will be. By adjusting the thickness of the p-SL, we hope to find a thickness that prevents mode leakage into the p-contact layer yet still maintains low impedance. 


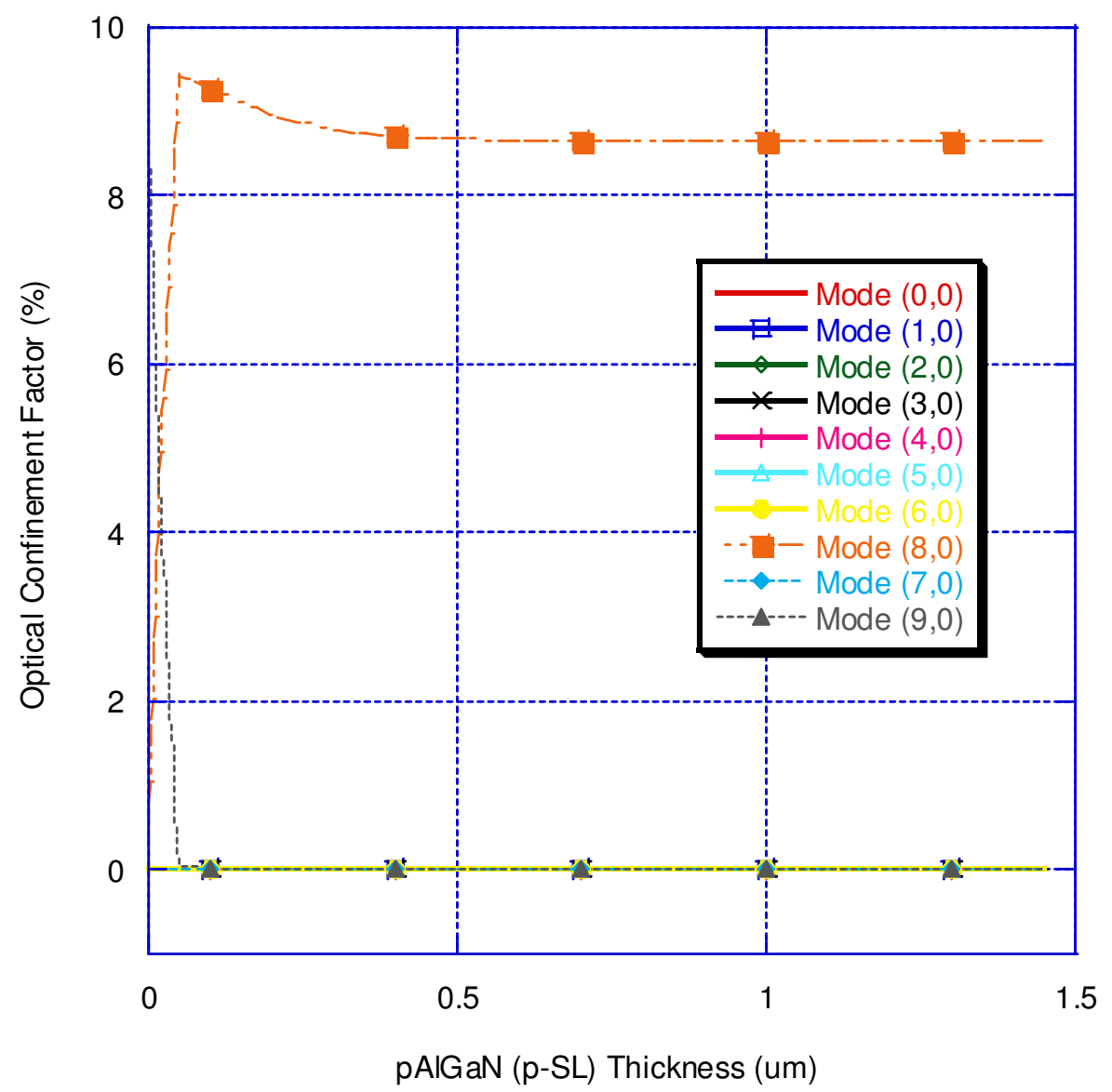

Figure 3-4: Optical Confinement vs. p-SL Thickness

The adjustment of the p-SL cladding layer shows a different increase in the OCF than the n-SL plot. It is interesting to point out that the thickening of the p-SL lattice above $0.1 \mu \mathrm{m}$ has a detrimental effect on the OCF, decreasing it from a maximum of $8.7 \%$ to $8.4 \%$. (It is important to note that the OCF is at a different mode). This is similar to the results in Figure 2-8 for the 1D design. Thus we find our optimal thickness for the p-SL that yields the best OCF still remains fairly thin is at $0.1 \mathrm{~nm}$. 


\section{4 n-GaN and p-GaN Cladding Thickness Adjust}

The GaN core layer in the 2D simulation has a refractive index greater than the surrounding cladding layers to reflect the light back towards the active region. A thicker core layer can lead to more optical losses due to the large area the light has to travel. Thus choosing an optimal core layer thickness is important in creating the most efficient laser diode.

The OCF is measured and plotted in Figure 3-5 for $n-G a N$ core layer thicknesses of $0-1 \mu \mathrm{m}$. The OCF peaks at $8.7027 \%$ with an $\mathrm{n}-\mathrm{GaN}$ thickness of $0.075 \mu \mathrm{m}$. This is a $0.224 \%$ increase in the OCF from $8.6762 \%$ when a $0.1 \mu \mathrm{m}$ layer is used. Thus by using a thinner layer of n-GaN core layer, a better optical confinement can be achieved. Figure 3-5 shows a constant decrease in the OCF as the $n-G a N$ thickness is increased and the lasing mode migrates to lower modes. 


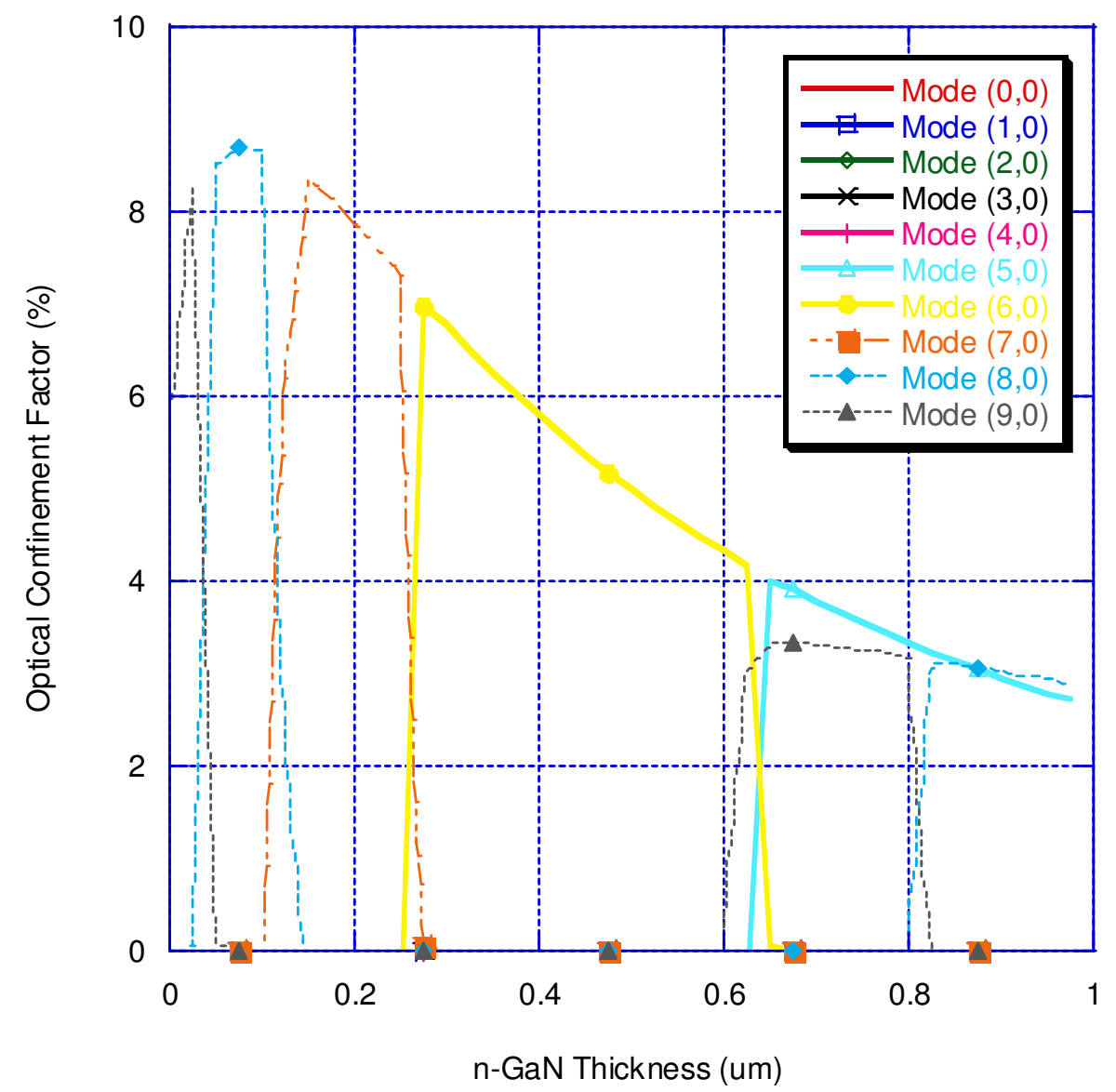

Figure 3-5: Optical Confinement vs. n-GaN Core Layer Thickness 2D

The same simulation was run for the $\mathrm{p}-\mathrm{GaN}$ core layer and the results of the OCF vs. p-GaN thickness are plotted in Figure 3-6. Figure 3-6 follows a similar trend to Figure 3-5. The maximum OCF is achieved for small thin layers of $\mathrm{p}-\mathrm{GaN}$. The OCF peaks with $8.8664 \%$ at a thickness of $0.05 \mu \mathrm{m}$. This is a $0.2 \%$ increase over the original design when the $\mathrm{p}-\mathrm{GaN}$ is $0.1 \mu \mathrm{m}$ thick and has an OCF of $8.6762 \%$. Similar to the $\mathrm{n}-\mathrm{GaN}$ core layer, a 
thinner layer creates better optical confinement. Both Figure 3-5 and Figure 3-6 are similar to the calculated results of Hatakoshi. ${ }^{12}$

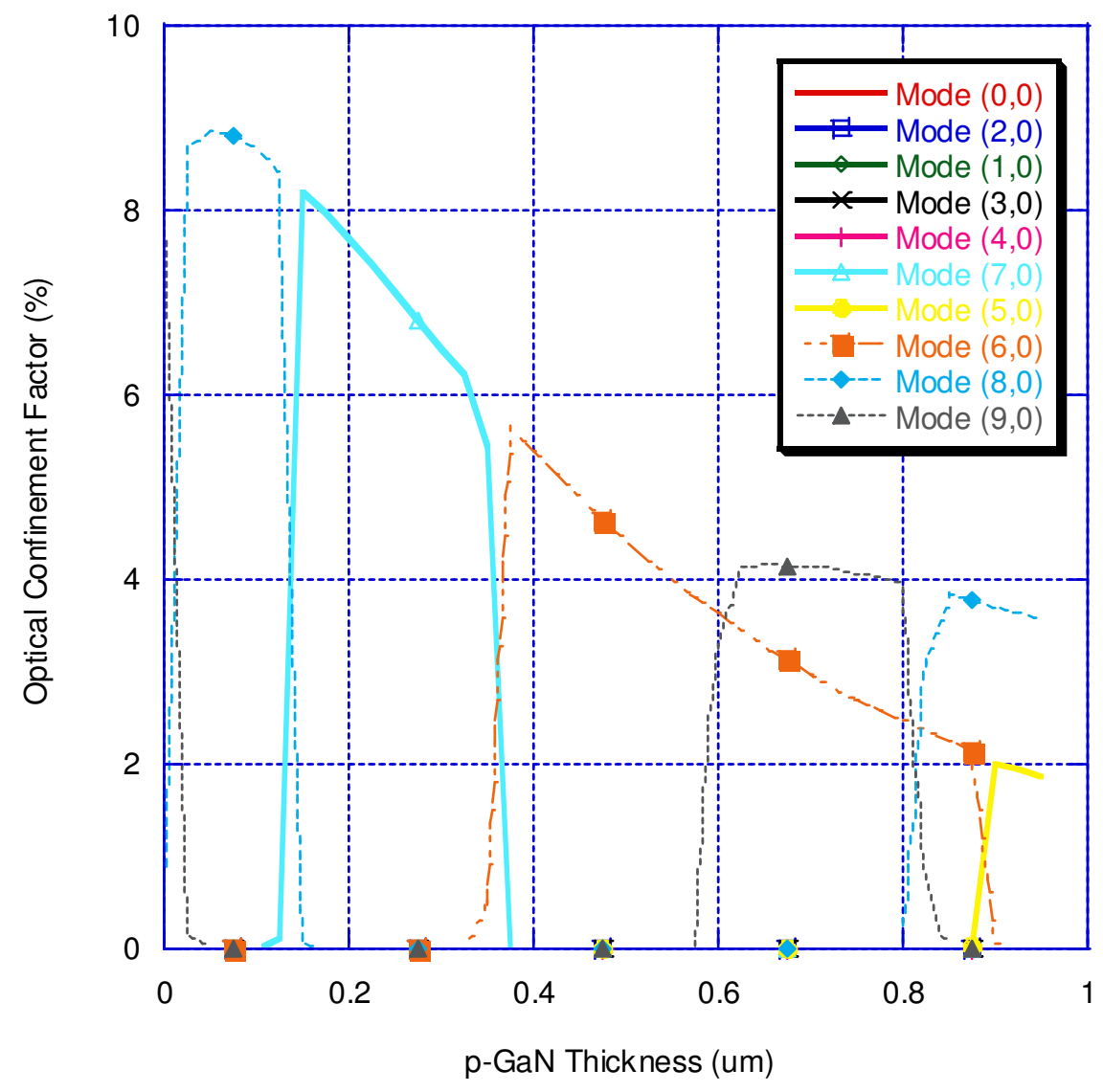

Figure 3-6: Optical Confinement vs. p-GaN Core Layer Thickness

\section{$3.51 D$ vs. $2 D$ Optical Modes}

It is very interesting to compare the $1 \mathrm{D}$ and $2 \mathrm{D}$ optical mode simulations. The important aspect that the 2D simulation provides that the 1D doesn't is the way the energy is centered along the $\mathrm{x}$-axis, while the modes vary along the $\mathrm{y}$-axis. 
Comparing the 1D and 2D simulations shows many of the same trends and similarities in the plots of the OCF, but do not have exact correlations on which thicknesses are the best for each layer. Instead there are certain layer thicknesses in the 1D design that maximize the $\mathrm{OCF}$ and certain layer thickness in the $2 \mathrm{D}$ design that maximizes the OCF. Inserting the air material at the top-right corner of the $2 \mathrm{D}$ design changes the characteristics of the laser-which mode the laser oscillates in and the OCF at this oscillating lasing mode. Thus although in the real world, actual realized LDs are 3D for the most part, the LaserMOD program provides us with a software that can bring many of the complexities occurring in the laser to a visual and mathematical interpretation in two dimensions. Although the designs don't yield the same exact numbers, the simulations still provide a reasonable reference for what's occurring inside the laser diode.

GaN laser diodes are special in that they have lasing in higher order modes above the fundamental mode ${ }^{4}$. Both the $1 \mathrm{D}$ and $2 \mathrm{D}$ simulations confirmed lasing in higher order modes. This is an important difference between normal laser diodes because lasing in the fundamental mode is more efficient as you don't get the loss from higher order modes, so creating a GaN laser that has similar efficiencies to a laser diode that operates at the fundamental mode can be challenging. In this thesis, changing layer thickness to optimize the laser diode is a simple yet necessary change that needs to be made to more efficiently confine the light.

The 1D simulation showed that the mode of operation is most affected by the GaN substrate layer thickness. The large thickness of the GaN substrate allows higher order modes to propagate and results in lost energy away from the fundamental mode. GaN 
laser diodes have a unique property that allows the lasing mode to occur at modes above the fundamental mode. The lasing mode has the highest OCF and the energy is located at the active region. Changing the thickness of other layers does not have as profound effects on the mode as changing the GaN substrate thickness.

The 2D simulation confirmed the results of the 1D simulation as well as [11]. The addition of another dimension causes changes in the way the program evaluates the waveguide calculations, but it still shows lasing at higher order modes. In fact, the lasing mode for the $2 \mathrm{D}$ is even higher than the $1 \mathrm{D}$ simulation. The $2 \mathrm{D}$ design also shares the same layer characteristics as the 1D simulation. Adjusting the thickness of any layer in $2 \mathrm{D}$ design shows the same effects as adjusting the layer in the 1D design. It is very important to have this consistency between the 1D and 2D designs because it shows validity in the theory of ghost modes and higher order lasing. 


\section{CHAPTER 4: OPTICAL POWER VS. CURRENT ANALYSIS IN 1D SIMULATION}

\subsection{Light vs. Current Plot of Original 1D Design}

Optimizing the OCF in the previous chapters for different layer thicknesses is very important in lowering the lasing threshold of the GaN LD. An increase in the OCF means that more light is being confined in the lasing mode. With more light focused in the lasing mode, less current will be required for the $\mathrm{LD}$ to achieve lasing. In this chapter, we demonstrate how the optimized OCF affects the Light vs. Current curves of the GaN LD. By creating a new laser design using our optimized thicknesses, we will see that it produces lower lasing thresholds.

QWs produce light by drawing an enormous amount of carriers into the wells which leads saturation. With a saturation of carriers in the well, it increases the chances of the recombination of a holes and electrons-which releases light. If we are to increase the number of quantum wells, the number of carriers must be increased to properly saturate each QW. In order to supply this increased number of carriers, a larger current must exist between the electrodes of the LD. ${ }^{16}$ Therefore we have shown that if we are to increase the number of quantum wells we expect a larger lasing threshold current. This phenomenon of increased lasing threshold for increase number of quantum wells is shown in Figure 4-1 and Figure 4-2. Figure 4-2 shows a zoomed in view of these lasing thresholds. These figures represent the Light vs. Current simulations for the 1D model. 


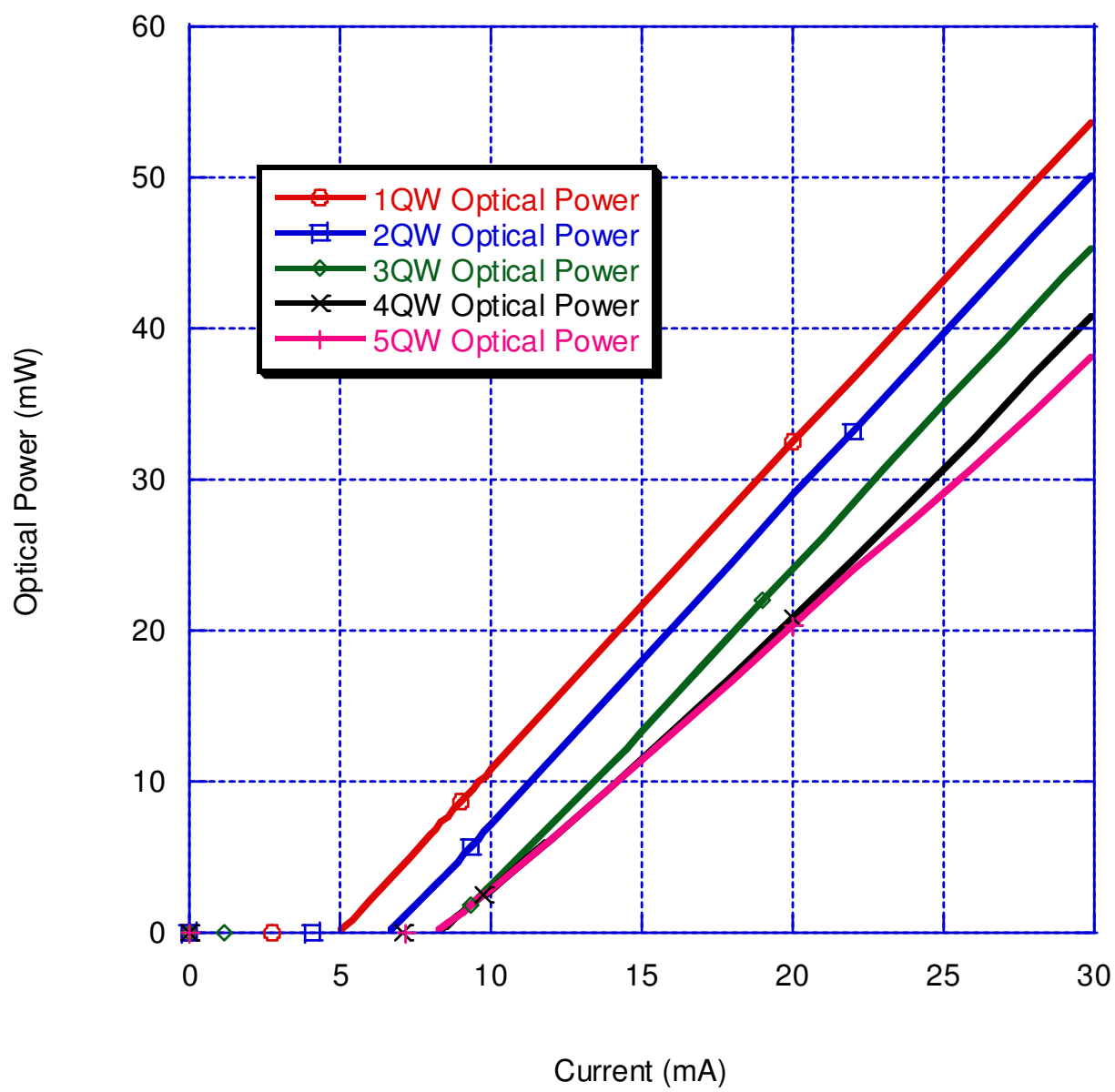

Figure 4-1: Optical Power vs. Current of the 1D Laser Diode 


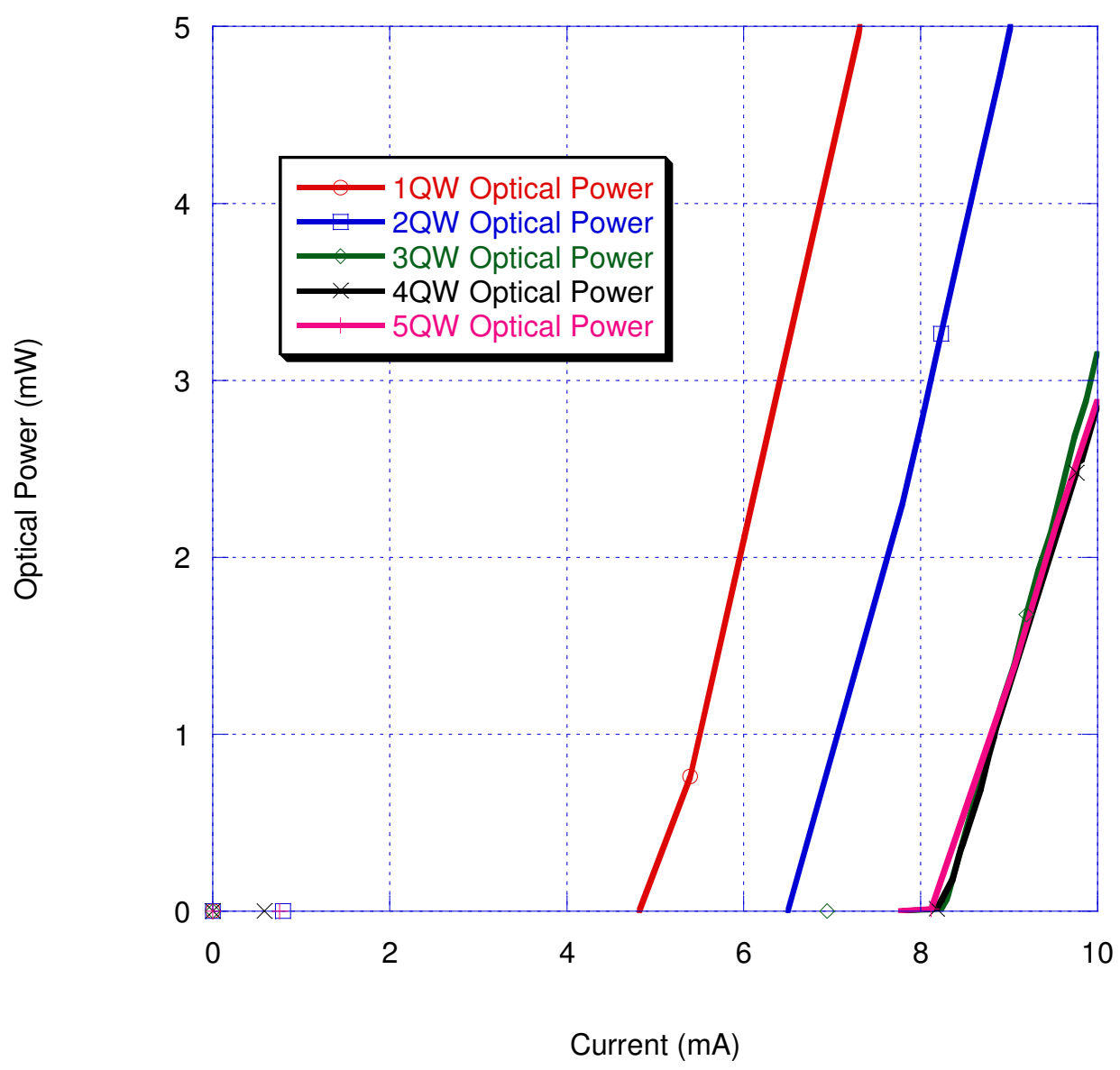

Figure 4-2: Threshold Currents view of Optical Power vs. Current of 1D Laser Diode

The lasing threshold increases as we increase the number of QWs from one QW to two QWs. With one QW the lasing threshold is $4.8 \mathrm{~mA}$ and when another QW is added the new lasing threshold is $6.5 \mathrm{~mA}$. Table $4-1$ shows the lasing thresholds for different number of QWs. When the QWs are increased to three, four and five QWs, the change in lasing threshold is very small. 
Table 4-1: Table of lasing threshold currents for different quantum wells

\begin{tabular}{|c|c|}
\hline Number of QWs & Lasing Threshold (mA) \\
\hline 1 & 4.8171 \\
\hline 2 & 6.4977 \\
\hline 3 & 8.2283 \\
\hline 4 & 8.1820 \\
\hline 5 & 8.1223 \\
\hline
\end{tabular}

\subsection{Light vs. Current Plot of Optimized 1D Design}

To optimize the lasing threshold of our GaN LD design, we use the optimal layer thicknesses calculated in Chapter 2. The new configuration can be seen in Table 4-2. The refractive indices remain the same, but the thicknesses of the cladding, core and substrate layers have changed. The design in Table 4-2 is implemented in LaserMOD and Light vs. Current simulation is run. 
Table 4-2: Optimized 1D laser diode design

\begin{tabular}{|c|c|c|}
\hline Layer & Thickness (nm) & Refractive Index (n) \\
\hline $\begin{array}{l}\text { p-GaN } \\
\text { (contact) }\end{array}$ & 50 & 2.55 \\
\hline $\begin{array}{c}\mathrm{p}-\mathrm{Al}_{0.12} \mathrm{Ga}_{0.88} \mathrm{~N} / \mathrm{GaN} \\
(\mathrm{p}-\mathrm{SL} \text { cladding) }\end{array}$ & 200 & 2.53 \\
\hline $\begin{array}{c}\text { p-GaN } \\
\text { (waveguide) }\end{array}$ & 50 & 2.55 \\
\hline $\begin{array}{c}\text { p- } \mathrm{Al}_{0.35} \mathrm{GA}_{0.65} \mathrm{~N} \\
\text { (e-block) }\end{array}$ & 20 & 2.42 \\
\hline $\mathrm{n}-\mathrm{GaN}$ & 15 & 2.55 \\
\hline $\begin{array}{c}\mathrm{In}_{0.1} \mathrm{GA}_{0.9} \mathrm{~N} / \mathrm{GaN} \\
(5 \mathrm{QWs})\end{array}$ & 67 & $2.685 / 2.55$ \\
\hline $\begin{array}{c}\text { n-GaN } \\
\text { (waveguide) }\end{array}$ & 75 & 2.55 \\
\hline $\begin{array}{c}\mathrm{n}-\mathrm{Al}_{0.12} \mathrm{Ga}_{0.88} \mathrm{~N} / \mathrm{GaN} \\
(\mathrm{n}-\mathrm{SL} \text { cladding })\end{array}$ & 800 & 2.53 \\
\hline $\begin{array}{c}\text { n-GaN } \\
\text { (Substrate or buffer) }\end{array}$ & 4100 & 2.55 \\
\hline $\begin{array}{l}\text { Sapphire } \\
\text { (Oxide) }\end{array}$ & 4000 & 1.77 \\
\hline
\end{tabular}




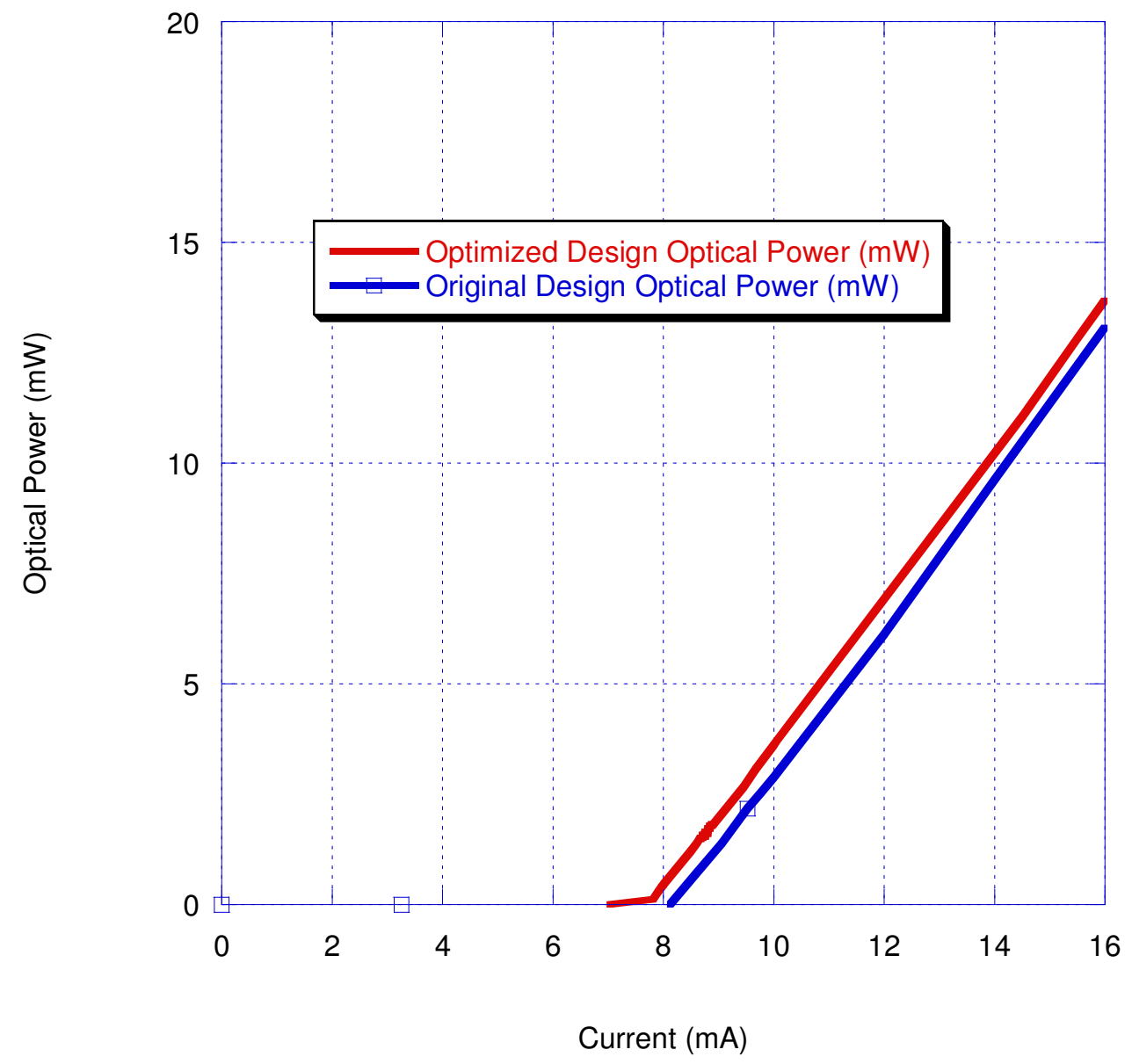

Figure 4-3: Lasing Thresholds of Original Design and Optimized Design

Figure 4-3 shows the Light vs. Current plot of the new optimized design and the original design. The new design has a simulated lasing threshold of approximately $7.8 \mathrm{~mA}$. This lasing threshold is $0.4 \mathrm{~mA}$ less than the original design. The significance of $0.4 \mathrm{~mA}$ should not be overlooked as it is almost a 5\% reduction in the lasing threshold 
which results in a $10 \%$ reduction in power dissipated. Thus the research and results are a success in that they provided us with the arrival of a more optimum design. By increasing the OCF a more efficient light output results and brings us one step closer to the ultimate goal in meeting the expectations of using laser diodes as a future light sources. 


\subsection{Conclusion}

Through simulating different designs we found a design that yielded an optimized OCF. With a larger OCF, the laser threshold is lowered and a more efficient laser diode is proposed. We use a systematic method of evaluating the optimum thickness of each layer, and are able to combine all the optimized layers to yield a single optimized design. In this thesis, we only adjust the thickness of layers, but for a deeper and more thorough analysis, it would be important to adjust other parameters as well. Carrier density, doping levels, alloy content, and placement of layers are further parameters besides layer thickness that could be evaluated for their effects on the OCF. Although OCF could be increased further, achieving dramatic effects by adjusting these parameters is not expected. However, similar to the systematic adjustment of the layer thickness, adjustment of these other parameters will yield consistent but small increases in the OCF. Therefore it remains of further research interest to find new materials and new designs that progress past the current OCF limitations.

During the research and simulation steps encountered in this thesis, important patterns are revealed. It is of further interest to study these patterns and explain why these patterns occurred. When adjusting the SL layers, there is a major difference in the effects of adjusting the n-SL layer versus the p-SL layer. Though the different lattices provide similar functions, their effects on mode and optical confinement are different. Possible experiments to study the effects of the lattice would include further simulations in which the alloy percentage content is adjusted. 
A deeper understanding of the waveguide modes in the $2 \mathrm{D}$ realm is presented. In higher modes of operation, the modal energy splits into dual modes of energy across the $\mathrm{x}$-axis. This is an interesting occurrence because it reveals the different ways that the light can oscillate in the GaN substrate layer. This observational perspective would not have been possible had we only done simulations in the 1D model. It would be of further interest to study if higher order modes contribute to lasing, and how the GaN substrate can be modified to prevent energy loss at higher order modes.

\subsection{Errors and Corrections and Future Work}

After performing the 1D and 2D simulations, it is discovered that the 2D simulations are designed to simulate symmetric boundary conditions that aren't realizable in real GaN laser diode construction. The symmetric boundary conditions affect my 2D results by causing the higher order modes to maintain a symmetric splitting energy in higher order modes. In the higher order 2D modes as shown in Figure 3-1 there is a splitting of the nodal energy, but this split shouldn't be perfectly symmetric as the results show. The lack symmetry in the layers along the x-axis should cause a lack of symmetry in the nodal energy. This error is a result of incorrectly modifying the material files for each layer. In future works it will be of interest to re-simulate the design with correct boundary conditions for each layer. Having correctly defined boundary conditions will ensure the materials in each layer interact properly. 


\section{BIBLIOGRAPHY}

[1] Nakamura, Shuji. The Blue Laser Diode. 2nd ed. Springer, 1997.

[2] Goldwasser, Samuel M. "Sam's Laser FAQ - Diode Lasers."

Sci.Electronics.Repair FAQ. 2008. 5 Mar. 2008

$<$ http://www.repairfaq.org/sam/laserdio.htm\#diocss0>.

[3] Neamen, Donald A. Semiconductor Physics and Devices: Basic Principles. 1992. $3^{\text {rd }}$ ed. New York City: McGraw Hill

[4] S. Einfeldt, S. Figge, T. Buttcher, and D. Hommel. "Coupling of optical modes in GaN based laser-diodes”. Physica Status Solidi@ 20030 (7): 2287-2291

[5] Schubert, E.F. "Slides on QWs and MQWs and SLs". October 2005. Rensselaer Polytechnic Institute. <http://www.ecse.rpi.edu/ schubert/>

[6] Piprek, Joachim. "Blue Laser Diodes." Optik and Photonik June 2007. 9 Nov. 2007 <http://www.wiley-vch.de/berlin/journals/op/07-02/OP0702_S52_S55.pdf>.

[7] Piprek, J., and S. Nakamura. "Physics of High-Power INGaN/GaN Lasers." IEEE 149 (2002): 146.

[8] RSOFT Design Group, Inc. LaserMOD v2.0 User Guide. 2004. New York: RSOFT Design.

[9] Wakita, Koichi. Semiconductor Optical Modulators. 1998. New York City: Springer, 1998.

[10] D. Botez, "Analytical approximation of the radiation confinement factor for the TE0 mode of a double heterojunction laser,” IEEE: Quantum Electronics, vol. QE14, pp. 230-232, 1978. 
[11] Hatakoshi G., Onomura M., Ishikawa M. "Optical, electrical and thermal analysis for GaN semiconductor lasers.” International Journal of Numerical Modeling:

Electronic Networks, Devices and Fields. 14 (2001): 303-323. 02 Dec. 2007.

[12] Hatakoshi, Gen-Ichi, Masaaki Onomura, Shinji Saito, Katsunobu Sasanuma, and Kazuhiko Itaya. "Analysis of Device Characteristics for InGaN Semiconductor Lasers." Japanese Journal of Applied Physics 38 (1999): 1780-1785. 16 Nov. 2007.

[13] Onomura, M., Saito, S., Sasanuma, K., Hatakoshi, G., Nakasuji, M., Rennie, J., Sugiura, L., Nunoue, S., Nishio, J., Itaya. "Analysis of Transverse Modes of Nitride-Based Laser Diodes." IEEE Journal of Selected Topics in Quantum Electronics 5 (1999): 765-770. 16 Nov. 2007.

[14] Bergmann, M J, and H C Casey, Jr. "Optical-field calculations for lossy multiplelayer AlGaN/lnGaN laser diodes." Journal of Applied Physics 84.3 (Aug. 1998): 1196-1203. 31 Jan. $2008<$ http://ap.aip.org/>. Path: Search.

[15] Thomson, John D., M. Yin, Susan V. Dewar, Peter Blood, A. Catrina Bryce, John H. Marsh, C. J. Hamilton, C. C. Button, and Peter M. Smotown. "The Effect of Cladding Layer Thickness on Large Optical Cavity 650-Nm Lasers." IEEE Journal of Quantum Electronics 38 (2002): 285-290. 16 Nov. 2007.

[16] K. Domen, K. Horino, A. Kuramata, and T. Tanahashi. "Gallium Nitride Materials, Processing, and Devices”, Digest of the LEOS Summer Topical Meetings 35 (August 1997) 
[17] X. Jin, B. Zhang, S. Jobe, J. DeLeon, J. Flickinger, T. Dai, G. Zhang, E. Heller, and L. Chen, "Two Dimension Simulation of Gallium Nitride-Based laser Diode", The $7^{\text {th }}$ International Conference on Numerical Simulation of Optoelectronic Devices (NUSOD 07), Delaware, United States, 24-27 September 2007.

[18] Xiaomin Jin, Zhang Bei, Dai Tao, Zhang Guo-Yi. "Effects of transverse mode coupling and optical confinement factor on gallium-nitride based laser diode." Chinese Physics B (2008): Volume 17, Issue 4, 1274-1279. 1 April 2008. 


\section{APPENDIX}

\section{Publishing Related to Thesis}

X. Jin, B. Zhang, S. Jobe, J. DeLeon, J. Flickinger, T. Dai, G. Zhang, E. Heller, and L.

Chen, "Two Dimension Simulation of Gallium Nitride-Based laser Diode", $\underline{\text { The } 7^{\text {th }}}$

International Conference on Numerical Simulation of Optoelectronic Devices (NUSOD

07), Delaware, United States, 24-27 September 2007.

X. Jin, B. Zhang, L. Chen, S. Jobe, T. Dai, and G. Zhang, "the Optimization of Gallium

Nitride-Based Laser Diode through Transverse Modes Calculation” The OSA Topical

Conference on Nanophotonics (NANO 2007), 6/18/2007 - 6/21/2007, Hangzhou, China.

Dennis Derickson, Sam Agbo, Sean Jobe, John Sharpe, Dan Wasche, and Xiaomin Jin,

Photonics Education Program at California Polytechnic State University”, Education and

Training in Optics and Photonics 2007 (ETOP 2007), Ottawa, Canada, June 2-3rd, 2007

Xiaomin Jin, Bei Zhang, Fei Wang, Jason Flickinger, Sean Jobe, Tao Dai, Guoyi Zhang,

"International Engineering Research and Educational Activity on GaN Lasers and LEDs"

International Association of Journals and Conferences (IAJC)-International Conference,

International Journal of Modern Engineering (IJME) IAJC-IJME 2008, November 18-22,

2008, Nashville, Tennessee. 
Xiaomin Jin, Sean Jobe, Simeon Trieu, Benafsh Husain, Jason Flickinger, Tao Dai, Bei Zhang, Xiang-Ning Kang, and GuoYi Zhang, "Mode Pattern Analysis of Gallium Nitridebased Laser diodes", The 3rd International Symposium on Photoelectronic Detection and Imaging (ISPDI 2009), Beijing, China, June 17 to 19, 2009. (Accepted) 